\title{
Human Body Structure Calibration Using Wearable Inertial Sensors
}

\author{
by \\ Xudong (Dora) Li
}

A thesis submitted to the Faculty of Graduate and Postdoctoral Affairs in partial fulfillment of the requirements for the degree of

Master of Applied Science

in

Human-Computer Interaction

Carleton University

Ottawa, Ontario

(C) 2017

Xudong (Dora) Li 


\begin{abstract}
This work proposes an inertial sensor-based human body structure parameters calibration methodology, aimed at being combined with a motion tracking and gesture recognition system in a virtual reality motion game context to reduce the player's motion control learning time and improve the accuracy and ease of game operation. This proposed calibration protocol is based on the three-axis accelerometer outputs by wireless inertial sensors to calibrate user's body parts length (including the forearm, upper arm, torso, shinbone, and whole leg) through four easy-to-perform static poses and a streamlined calibration procedure. Through this experiment, this calibration methodology proved to be a robust approach to calibrate physically normal users' body parts length, with satisfactory calibration accuracy (overall $7.64 \%$ average calibration error rate). In addition, in order to make the calibration process more efficient, effective, and user-friendly, a calibration auxiliary system sample interface to facilitate users has been proposed in this thesis.
\end{abstract}




\section{Acknowledgements}

I would like to recognize and thank my supervisor, Anthony Whitehead, for offering me this valuable thesis opportunity, and for providing me detailed and enlightening guidance throughout this process. I would also like to express my gratitude to my comrades for the help they have offered me. I also would like to sincerely thank my family and friends, who have constantly provided me with emotional and life support during this research so I could fully devote myself to thesis research and writing. 


\section{Table of Contents}

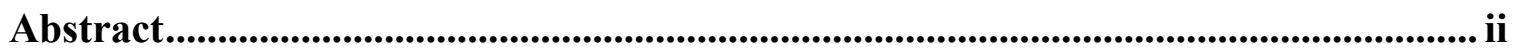

Acknowledgements ................................................................................................................ iii

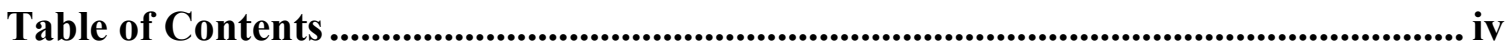

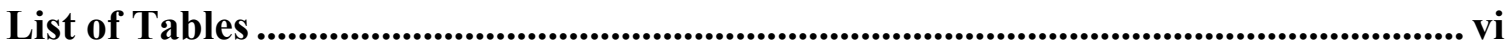

List of Figures.............................................................................................................................. vii

List of Appendices.............................................................................................. viii

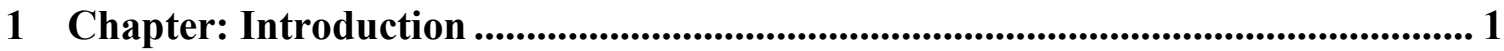

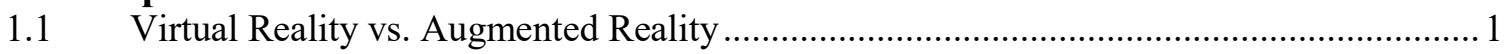

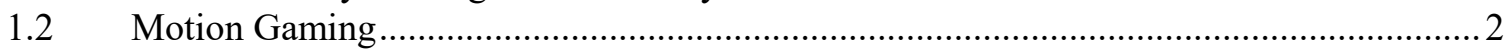

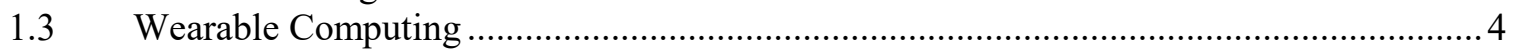

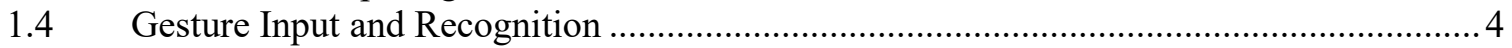

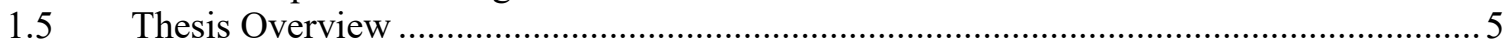

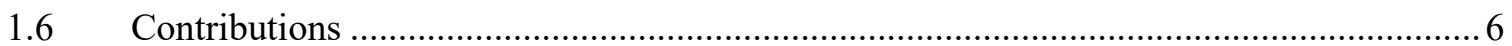

2 Chapter: Background and Related Works........................................................ 8

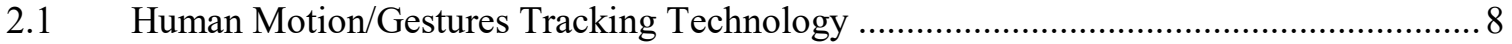

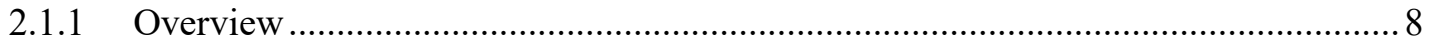

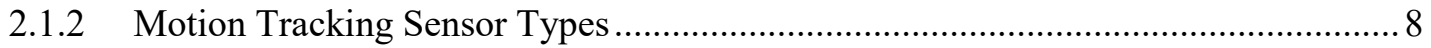

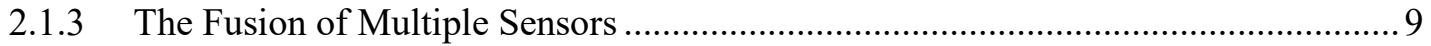

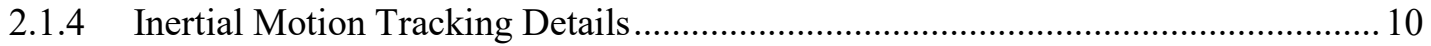

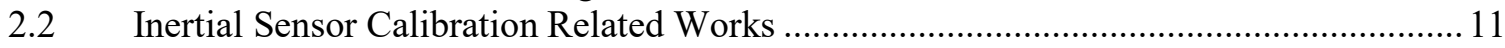

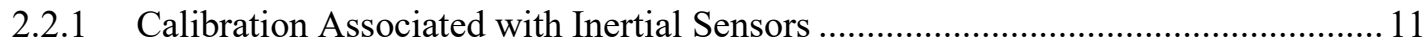

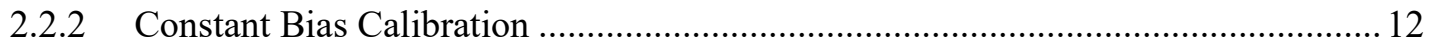

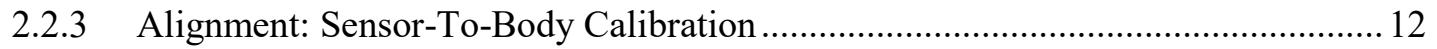

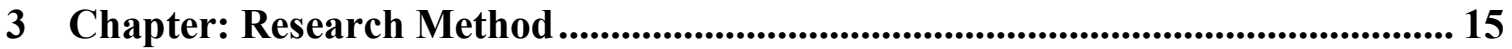

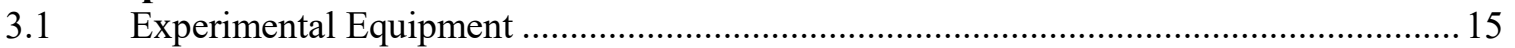

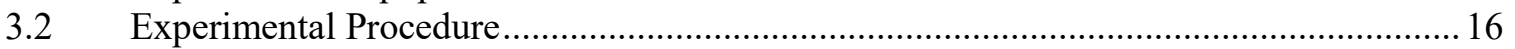

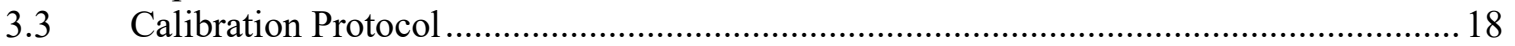

3.3.1 Pose 1 Forearm Length Calibration Protocol ............................................................. 18

3.3.2 Pose 2 Shinbone Length Calibration Protocol ........................................................ 19

3.3.3 Pose 3 Whole Leg Length Calibration Protocol....................................................... 22

3.3.4 Pose 4 Upper Arm and Torso Length Calibration Protocol ....................................23

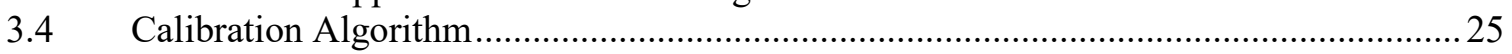

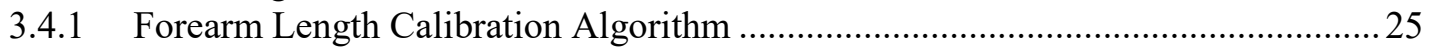

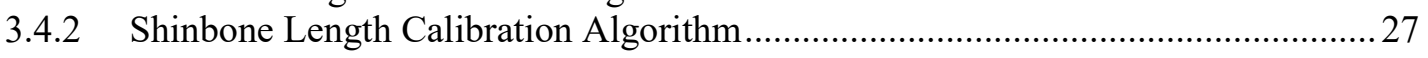

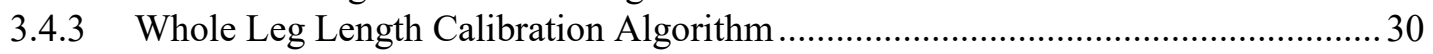

3.4.4 Upper Arm and Torso Length Calibration Algorithm......................................... 31

4 Chapter: Results Analysis .............................................................................................. 35

4.1 Average Calibration Accuracy ................................................................................... 35

4.2 Comparison of Calibration Accuracy between Left and Right Body Sides...................... 37

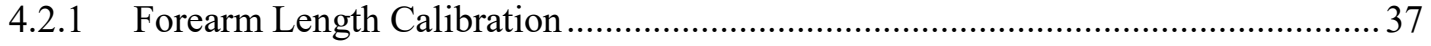

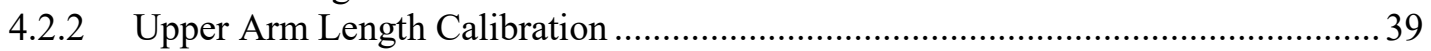

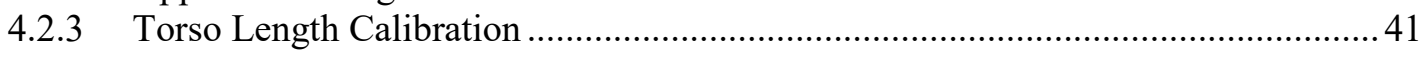

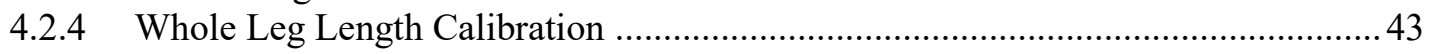




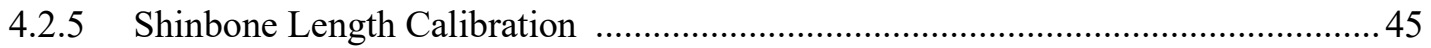

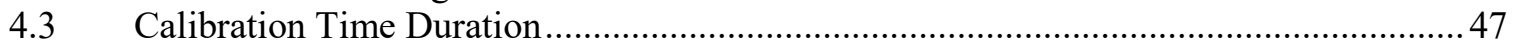

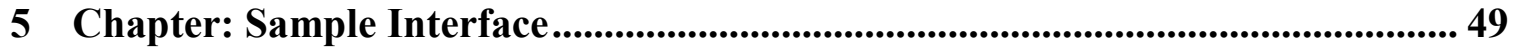

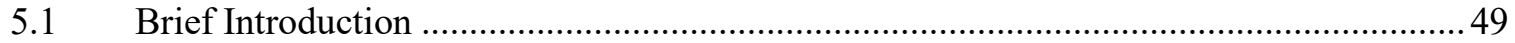

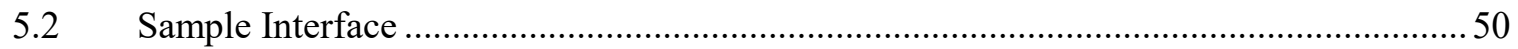

5.2.1 Welcome and General Introduction Sample Interface ...........................................50

5.2.2 Pose 1 Forearm Length Calibration Sample Interface ............................................51

5.2.3 Pose 2 Shinbone Length Calibration Sample Interface ............................................52

5.2.4 Pose 3 Whole Leg Length Calibration Sample Interface ......................................5

5.2.5 Pose 4 Upper Arm and Torso Length Calibration Sample Interface.........................55

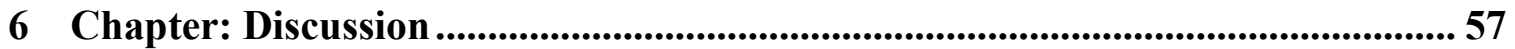

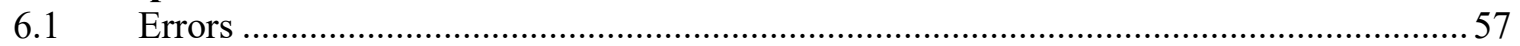

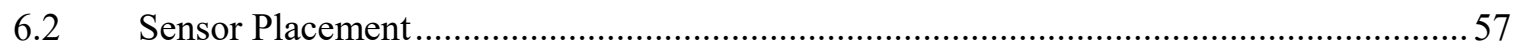

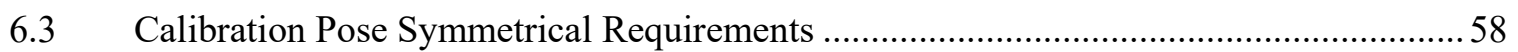

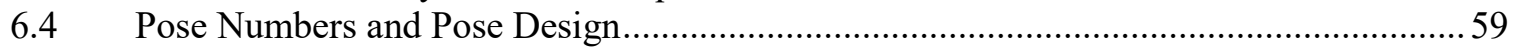

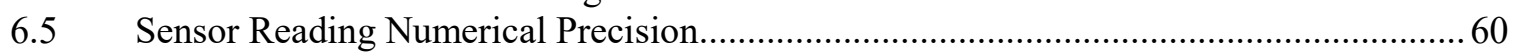

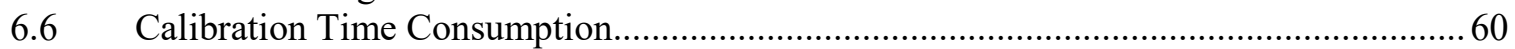

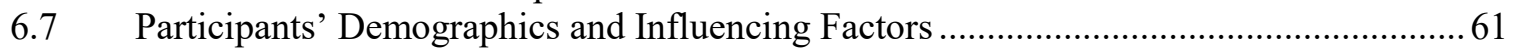

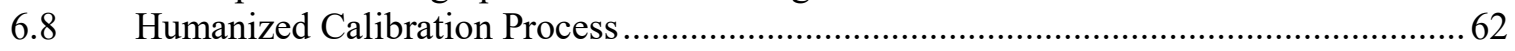

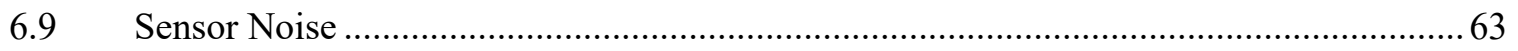

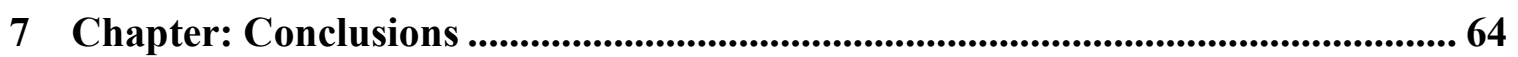

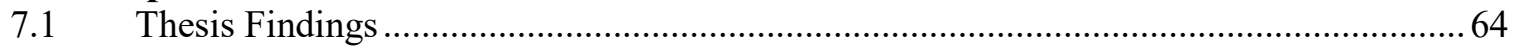

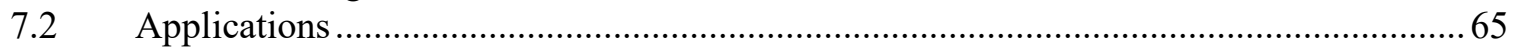

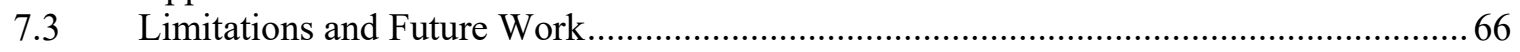

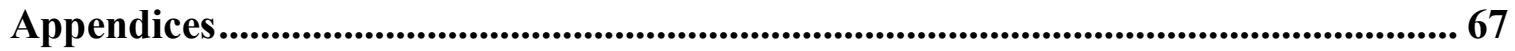

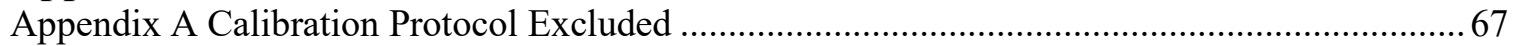

A.1 Pose for Calibration Shinbone Length in Situation of Knees Can Touch Together 67

Appendix B Calibration Algorithm Excluded and Supplementary Explanation ........................... 69

B.1 Calibration Algorithm for Shinbone Length in the Situation Where Knees Can

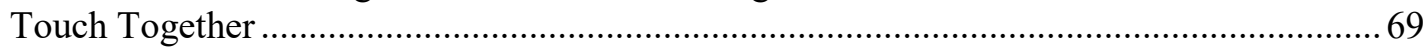

B.2 Verification of Combining Algorithm of Shinbone Length for Both Situations:

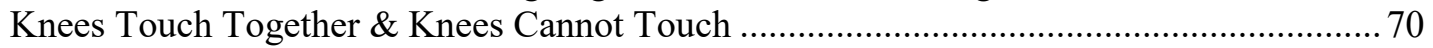

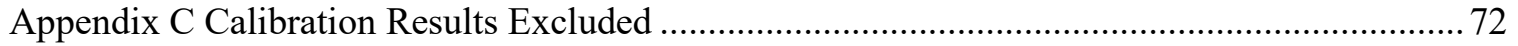

C.1 Shinbone Length Calibration Results Excluded....................................................... 72

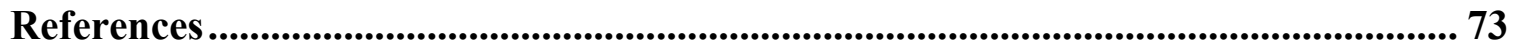




\section{List of Tables}

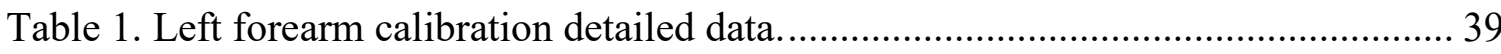

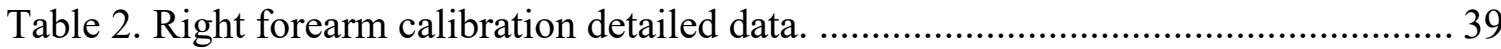

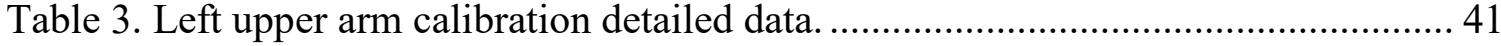

Table 4. Right upper arm calibration detailed data...................................................... 41

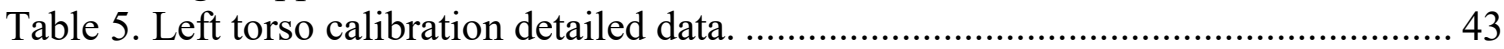

Table 6. Right torso calibration detailed data. ............................................................. 43

Table 7. Left whole leg calibration detailed data........................................................ 45

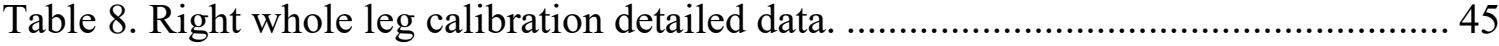

Table 9. Left shinbone calibration detailed data.............................................................. 47

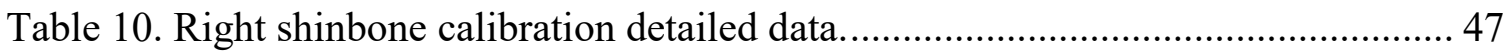

Table 11. Shinbone calibration detailed data for P8 .................................................. 72 


\section{List of Figures}

Figure 1. The outward appearance and the inside circuit board of Texas Instruments CC2650STK, and its accompanying wireless mobile data monitor platform. ................ 15

Figure 2. The directions of its global axes of CC2650STK used in this research........... 16 Figure 3. The three-perspective simulating figure to illustrate the calibration pose for calculating forearm length.

Figure 4. The three-perspective simulating figure to illustrate the calibration pose for calculating the shinbone length.

Figure 5. The three-perspective simulating figure to illustrate the calibration pose for calculating the whole leg length.

Figure 6 . The three-perspective simulating figure to illustrate the calibration pose for

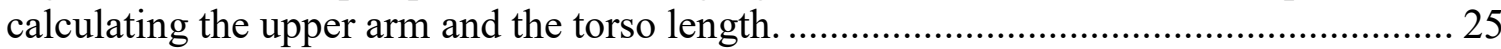

Figure 7. Skeletal model for explaining calibration algorithm for forearm length........... 27

Figure 8. Skeletal model for explaining calibration algorithm for shinbone length......... 29 Figure 9. Skeletal model for explaining calibration algorithm for whole leg length........ 31 Figure 10. Skeletal model for explaining calibration algorithm for upper arm and torso

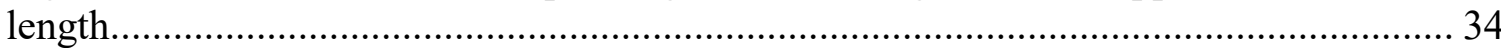

Figure 11. Calibration average error rates for different body parts. ............................. 36 Figure 12. Individual participant's calibration error rates for different body parts......... 36 Figure 13. Forearm calibration average error rates for the left and right sides. .............. 38 Figure 14. Forearm calibration error rates for individual participants on the left and right

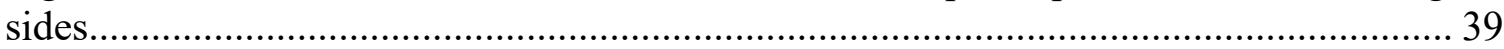

Figure 15. Upper arm calibration average error rates for the left and right sides........... 40 Figure 16. Upper arm calibration error rates for individual participants on the left and right sides.

Figure 17. Torso calibration average error rates for the left and right sides.................. 42

Figure 18. Torso calibration error rates for individual participants on the left and right

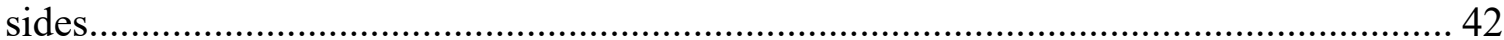

Figure 19. Whole leg calibration average error rates for the left and right sides. ........... 44 Figure 20. Whole leg calibration error rates for individual participants on the left and

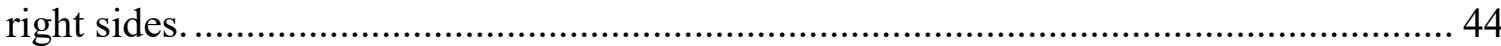

Figure 21. Shinbone calibration average error rates for the left and right sides.............46 Figure 22. Shinbone calibration error rates for individual participants on the left and right

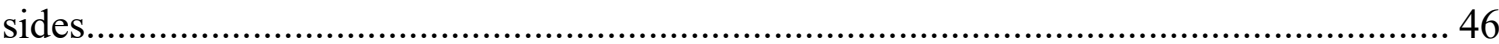

Figure 23. Total calibration time duration for individual participants........................... 48

Figure 24. Average calibration time proportion for different body parts. ...................... 48

Figure 25. Calibration system introduction sample interface. ....................................... 50

Figure 26. Pose 1 forearm length calibration system sample interface. ........................ 52 Figure 27. Pose 2 shinbone length calibration system sample interface......................... 53

Figure 28. Pose 3 whole leg length calibration system sample interface. ....................... 55

Figure 29. Pose 4 upper arm and torso length calibration system sample interface......... 56

Figure 30. Illustration for symmetrical requirements of calibration pose performing. .... 59 Figure 31. The three-perspective simulating figure to illustrate the calibration pose for

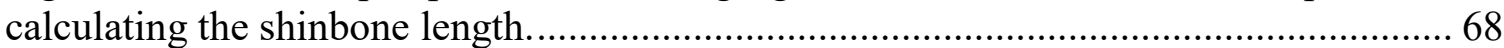

Figure 32. Skeletal model for explaining calibration algorithm for shinbone length...... 70 


\section{List of Appendices}

Appendix A Calibration Protocol Excluded ................................................................ 67

A.1 Pose for Calibration Shinbone Length in Situation of Knees Can Touch Together 67

Appendix B Calibration Algorithm Excluded and Supplementary Explanation .............. 69

B.1 Calibration Algorithm for Shinbone Length in the Situation Where Knees Can

Touch Together

B.2 Verification of Combining Algorithm of Shinbone Length for Both Situations:

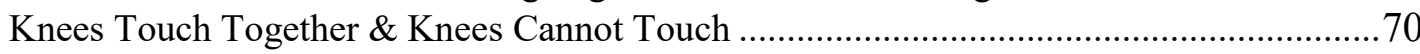

Appendix C Calibration Results Excluded ............................................................... 72

C.1 Shinbone Length Calibration Results Excluded................................................... 72 


\section{Chapter: Introduction}

In the last decade, thanks to the new technological advances in low-cost, miniature and lightweight trends, many have been inspired to open their minds and think about what alternative and innovative approaches, other than just desktops and laptops, that a computational device could use to free users' hands from the keyboard, mouse, and even touch screen, which could allow for a more natural and intuitive interactions with devices to enhance and enrich the user experience, and also could better facilitate and improve operational enjoyment. In this new trend, various novel interaction modalities have emerged and shown their promising prospects. Among them, there is no doubt that wearable computing is one of the most popular, which allows convenient interaction with users based on their daily life context to intelligently and effectively assist their activities, and is promising in tremendous practical applications, for example, augmented reality, motion controlled games, etc.

Sensing techniques, such as inertial sensors, could boost the development of wearable computing, virtual reality, motion tracking, and gesture recognition technology. Calibration is an important stage of applying inertial sensors, and always possesses significance for the performance and effectiveness of motion controlled games and virtual reality which leverage inertial sensors. Therefore, in the context of virtual reality plus motion tracking and gesture recognition technology as well as motion controlled games, constantly optimizing and developing calibration methodology for inertial sensors to minimize errors and increase accuracy is of great significance.

\subsection{Virtual Reality vs. Augmented Reality}

Both virtual reality and augmented reality are popular topics in new technology development, and they are drawing attention worldwide and are promising in their practical applications.

Virtual reality, typically realized by wearing a headset, immerses users in a recreated real life situation simulation through computer-generated vision and sounds to make users feel as if they were experiencing the real life environment. For example, Oculus Rift is a virtual reality headset developed by Oculus VR (a department in Facebook Inc.). 
By releasing their development kits to the public and the Kickstarter campaign, Oculus VR has attracted tremendous attentions from worldwide small and big companies, which has led to a massive interest in developing immersive virtual reality, fun-to-use motion controls, and also provided new opportunities for gaming design.

Unlike virtual reality, augmented reality layers computer-generated enhancements on top of the existing real life scene to provide additional information and instructions, which alters the experience that how the real world and digital information could interact with one another, and facilitates users to achieve goals and assist in their life. For example, Microsoft HoloLens can map the environment through its embedded sensors, without help from any outside positioning equipment, and generates a genuinely standalone holographic vision. In addition, more and more augmented reality applications have been developed in mobile devices, such as smartphones, tablets, and laptops.

Therefore, both virtual reality and augmented reality are aimed at enhancing and enriching users' experience.

\subsection{Motion Gaming}

For a long time, button-based controllers have dominated the gaming input standard modality. However, in the last decade, motion gaming, as a new trend, has emerged in gaming control technology and shows exciting prospects. Motion game is also called the motion-controlled game, and players could control and interact with the game through the combination of their body movements and spoken commands.

Motion controlled gaming provides the opportunities which allow players to exercise while playing the video game; therefore, it is also an exergame. In contrast to button based video game controls which could potentially cause physical fatigues and damages if deeply addicted to the game, motion controlled gaming would be more healthy and beneficial to players. Research has reported the tendency toward more and more extensive health problems within the general population, such as weight issues, obesity, and heart disease, that have direct or indirect relationships with the prevalent sedentary lifestyles [1]. Therefore, motion gaming has been seen as a promising approach to 
encourage players toward more exercise to tackle and prevent these health problems in a fun way.

Thanks to the technological advances in low-cost, miniature, lightweight sensor device which enables and boosts the development of motion controlled gaming [2]. The Nintendo Wii has achieved tremendous success because it was the first released to market video gaming system leveraging motion controlled technology, which allows the player to hold a hardware controller in hand to perform body movements to control the game. The mechanism behind Wii Remote motion controlled gaming is that the game console equipped with a camera to track the positon of the hardware controller, and the remote game controller contains accelerometer device and infrared sensors which will detect and wirelessly report detailed information about the player's movements to the game console [2]. Through the above system architecture, Nintendo Wii can track the player's body movements information for the control system input, such as various movement types, direction and angular degree, acceleration, etc.

However, there are innate drawbacks in Nintendo Wii in that Wii could only recognize simple wrist flicks rather than full body motions, which would reduce the players' passion for performing full body movements while playing because they can simply sit on the couch and perform simple upper limb motions [3].

In contrast to Nintendo Wii, Kinect, a camera-based motion gaming system has been released by Microsoft, which is more effective in encouraging players to perform full body motions because it tracks players' full body [3] instead of just a certain body part, and it requires players to perform full body movements while playing in order to succeed and achieve better score in the game. Trying to perform minimal body motions would lead to unsatisfactory scores or even failure in the game. Therefore, despite its issues with latency errors, being sensitive to lighting conditions [3], etc., Kinect possesses advantages in encouraging players to thoroughly exercise while playing.

With the development of science and technology, more and more innovative sensing techniques have presented their promising prospects to be incorporated into motion gaming, for instance, inertial sensors [3], mind-machine interfaces [4], [5], and biological sensors [6], [7], [8], [9], which would all boost the bright future of motion gaming development. 


\subsection{Wearable Computing}

Wearable computing refers to the technology practice based on computational and sensor devices worn on users' body (under or over clothing), such as wearing eyeglasses, a watch, or clothing. To date, there is no doubt that wearable computing technology is one of the most popular innovations, a substantial literature has emerged on this topic, and many companies have paid considerable attention to developing and selling wearable computing applications. The idea of wearable computing application inspires people to consider alternative and innovative ways that a computer could be used to facilitate daily life, other than just desktops and laptops. Wearable computing is one of the novel approaches that computational devices could conveniently interact with users based on their daily life context to intelligently and effectively assist their activities. Wearable computing technology encompasses a wide range of potential uses that are under investigation, including (but not limited to) healthcare, training, security applications [10], and video games [11]. Especially in the field of healthcare and clinical applications, due to its inexpensive, convenient, flexible, efficient, portable, and adaptable advantages [12], [13], wearable computing technology is applied successfully and shows great prospects in gait analysis to provide valuable assessment information for numerous health-related applications, such as rehabilitation and diagnosis of medical conditions [12], to help clinicians to measure patients' evolution [14], and determine the optimal care level they should receive [15].

\subsection{Gesture Input and Recognition}

User gestures, typically composed of motions expressed by hands [16], are an important aspect of human-computer interactions [2]. Gestures are often used for commands input for a computing device to enhance its efficiency and ease of use. In order to release users' hands from a keyboard, mouse, and even touch screen, gestures input and recognition are deemed a promising approach because they allow more natural interactions with devices, more intuitive controls in a $3 \mathrm{D}$ virtual environment, and also make it more convenient for physically impaired users to access computing devices.

To realize gesture control, a gesture recognition algorithm system is a key 
component. The essential purpose of human gesture recognition is to develop a system which can efficiently and accurately identify human gestures to convey information and eventually realize intuitively interactive control over systems, such as, PCs, TVs, or mobile devices [2], [16]. Gesture recognition has a wide range of applications, for example, video games control [17], applications on mobile phones [18], augmented reality [19], surgical navigation [20], and monitoring patients' emotional states or stress levels [21]. In terms of recognition methods, there are two main types: vision-based gesture recognition, and using accelerometers and/or gyroscopes [16]. For vision-based gesture recognition, it can be realized by pattern matching approaches [22], [23], although it might consume more processing time [24], and might suffer from unexpected image noise [16]. For the gesture recognition method leveraging accelerometers or/and gyroscopes, its merits are to detect and resolve fast user motions and can be used as a standalone input device [25].

\subsection{Thesis Overview}

This thesis is organized as follows.

Chapter 1 and Chapter 2 presents the background introduction and related works related to this thesis topic, including the overview of human motion and gesture tracking technology, motion tracking sensor types, the fusion of multiple sensors, the detailed introduction about inertial motion tracking, and the inertial sensor calibration related works, such as error types, calibration about constant bias, and calibration about alignment.

Chapter 3 introduces the research method in this thesis, involving the experimental equipment (sensors, accompanying equipment and platform, etc.), experimental overall procedure, the proposed calibration protocol (sensor placement, calibration poses and requirements, etc.), and the calibration algorithm.

Chapter 4 presents the experimental results to understand the utility, advantages, and disadvantages of this proposed calibration methodology, including a brief explanation of the key evaluation indicator used in this thesis, the average calibration accuracy across all participants, the calibration accuracy for different body parts and for individual participants, the calibration accuracy comparison between the left and right body sides, and the calibration time duration. 
Chapter 5 introduces a calibration auxiliary system and a set of sample interfaces for this proposed calibration methodology, which involves all the required poses and requirements designed in this thesis. Especially, the Pose Monitor system proposed in Chapter 5 is aimed at facilitating users to intuitively understand the pose requirements and conveniently evaluate their own pose through the dynamical and real-time reflection in the interface, so they could adjust their pose by themselves.

Chapter 6 discusses the findings and thoughts about the proposed calibration methodology in more depth from various aspects, involving errors, sensor placement, calibration pose design and requirements, sensor reading numerical precision, calibration time consumption, etc., and Chapter 7 summarizes the findings of the thesis, promising applications, as well as limitations and future work.

\subsection{Contributions}

Although massive amounts of research have been done in the past few years on inertial sensors calibration, the majority of these works have focused on addressing one or more of the following issues of inertial sensors in motion tracking and gesture recognition system: the deterministic errors, stochastic errors [26], and alignment errors [27]. No study to date has provided a calibration protocol specifically designed to calibrate human body structure parameters (main body segments' length) using inertial sensors.

In this thesis, an inertial sensor-based human body structure calibration methodology has been presented. This proposed calibration protocol is based on the accelerometer outputs from the wireless inertial sensors to calibrate users' body parts length (including the forearm, upper arm, torso, shinbone, and whole leg) with simple calibration poses design and streamlined testing procedure. This calibration method would be expected to be combined with inertial sensor-based motion tracking and gesture recognition system in a virtual reality motion game context. Through inputting player's body segments length as parameters into the virtual reality motion game system to personalize and customize the body structure parameters of virtual game character the same with player's, this proposed calibration methodology could be expected to help reduce the 
motion control learning time required for players, enhance the efficiency of adapting to game control, and improve the accuracy and ease of game operation.

In this research, the effectiveness of this proposed inertial sensor-based human body structure calibration methodology also has been tested and proved to be robust with satisfactory calibration accuracy and stability.

In addition, in order to make the calibration process more efficient, effective, and user-friendly, this thesis also proposes a calibration auxiliary system and a set of the sample interface, including a Pose Monitor interface, which can dynamically track users' poses to evaluate if their pose meet the requirements or not, and can facilitate users to intuitively understand the requirements of each pose and conveniently evaluate their own pose performing through the dynamical and real-time reflection in the interface, so users could feel more informed, confident, friendly, and could adjust their pose performance by themselves to meet the requirements. 


\section{Chapter: Background and Related Works}

\subsection{Human Motion/Gestures Tracking Technology}

\subsubsection{Overview}

The prosperous development of technology and science has made human-computer interaction a ubiquitous activity in daily life [28]. Human motion tracking technologies, with the intent to track human motion and convey the information to the computing device as input instructions, have drawn much attention in the past decade [29]. Human motion and gestures are expressive, meaningful body movements aimed to convey meaningful information to the environment or to communicate with others, which may involve physical motions of the fingers, hands, arms, head, or full-body [21]. At present, a large number of human motion tracking systems is commercially available for use [30].

\subsubsection{Motion Tracking Sensor Types}

In past decades, various human motion and gesture tracking technologies have been presented [31], mainly relying on mechanical, magnetic, acoustic, inertial, optical, radio, or microwave sensors to measure and generate signals to track and recognize user motion and gestures [32], [33], [16]. Each sensor has its advantages and drawbacks, as follow:

Mechanical Sensor: It can accurately estimate motion and gestures with a low latency, but usually with large volume, so it is hard to be mobile and portable.

Magnetic Sensor: It possesses accurate estimation of motion and gestures with a low latency and good mobility [34]. But it is easily affected and distorted by conductive objects in the environment, and sensor's signals reduce quickly when moving away from the magnet [31].

Acoustic Sensor: It has good mobility because of small in size and lightweight, but vulnerable to background environmental noise and atmospheric influencing factors, which might easily affect sensor's accuracy [31].

Inertial Sensor: Its advantages are lightweight, excellent ability to capture fast motion and gestures, and sensing range is large, but due to zero drift issue, its long-term stability might be a concern [31]. 
Visual Sensor: Its motion and gesture recognition accuracy is generally higher because it directly extracts features from images [35] with little accumulated errors [36], but it lacks the ability to track fast movements because of motion blur and occlusions, which may reduce its efficiency of feature extraction, and may influence its real-time motion tracking ability [35].

Radio and Microwave Sensor: Its mobility is superb and sensing range is large, but with poor tracking precision [32].

\subsubsection{The Fusion of Multiple Sensors}

In terms of motion and gesture recognition, most of the sensor technologies mentioned above present satisfactory performance when used alone, especially when not requiring real-time recognition [37], [38]. However, when tracking motion and gestures, due to their innate limitations, such as volume, mobility, accuracy, noise, latency, or tracking range, none of the sensors is capable of perfectly tracking motion and gestures on its own [29], [31].

Therefore, in order to overcome the innate disadvantages of each individual sensor and better track human motion and gestures, numerous studies have proposed several approaches to combine multiple sensors [21], some examples are as below:

Inertial Measurement Units (IMUs): This is the typical motion tracking system used in many labs [39], [40], [41], [42], which aims at tracking positions and directions of target objects through integrating multiple sensors, involving an accelerometer, a gyroscope, and a magnetometer.

Visual and Inertial Sensor Fusion: This refers to the hybrid tracking system based on inertial sensors and vision sensors [43], [44], [45]. In paper [29], the authors present the $\mu \mathrm{IC}$ system, which integrates inertial measurement units (IMUs) and a web-based camera. By fusing these two sensors, the hybrid tracking system can overcome the drawbacks of each sensor to enhance the overall tracking performance, which is to say that visual measurements can support to correct the drift problem of inertial sensor, and inertial measurements can help to track and estimate motions and gestures when images from the visual sensor are blurred or lost due to quick movements and occlusions [29], [31]. The 
fusion of measurements from inertial sensors and vision system is most widely implemented by using the Kalman filter [31], [46], [47].

GPS and Inertial Sensor (or Visual Sensor) Fusion: In recent years, there has emerged an increasing interest in exploring this topic [48], [49], [50], and researchers even expand their research scope on this topic through involving more sensors types, such as velocity meters, and barometers [51], [52], to explore more combination possibilities to increase tracking accuracy.

Acoustic and Visual Sensor Fusion: The acoustic sensor helps visual sensor to track motions when the object is occluded, and visual sensor supports acoustic sensor's limitation in the multiple objects tracking situation [31]. Multiple objects tracking has been achieved through this sensor fusion strategy [21].

\subsubsection{Inertial Motion Tracking Details}

Inertial motion tracking, typically based on Microelectromechanical systems (MEMS)-based inertial measurement units (IMUs), is the process of locating target's current position based on its earlier position through analyzing integrated inertial sensor data [27]. IMUs, comprising a combination of accelerometers, gyroscopes, and magnetometers, can be conveniently attached to body segments of interest [30], [14], and IMUs are the popular choice for human motion capture, feature extraction [53], [54], [55], and motion evaluation [56], [57] due to their merits of lightweight, reduced size, portability, low cost, excellent ability to track fast motions, high response frequency, wireless connectivity [30], high flexibility and adaptability [12], [13], and independence from outside signals [35]. Therefore, considering its valuable features, IMUs have been regarded as a promising alternative for the camera-based tracking systems [58], [59], [60] which limit the workplace to a camera equipped room [61]. Since IMUs is wearable, flexible [62], [63], so it can perfectly support motion tracking in daily life context (indoor or outdoor environments). However, due to the zero drift problem, IMUs long-term stability might be a concern [35]. 


\subsection{Inertial Sensor Calibration Related Works}

\subsubsection{Calibration Associated with Inertial Sensors}

Studies have demonstrated that the calibration stages of the inertial sensors influence the accuracy of the angular rates and accelerations in motion tracking system [29], [14]. To prevent degrading the performance of inertial sensors, careful calibration is needed to properly model and compensate the errors and noises of inertial sensors.

Among all sources of errors related to inertial sensors, deterministic errors, stochastic errors [26], and calibration errors [27] are the prevailing error types. Deterministic errors involve constant biases and scale factors, and can be directly removed from measurements [26] (please see [64], [65] for details and examples about deterministic errors calibration of inertial sensors). Stochastic errors include angle/value random walk, unstable bias, etc. [66], which require modeling to address this issue because this type error cannot be directly removed from the measurements [29] (please see [66], [67], [68] for details and examples about stochastic errors identification). In addition, calibration errors are referred to the alignment issues, which may occur when aligning the frames of reference of inertial sensors and human limbs [30], as well as aligning the frames of reference of different inertial sensors.

However, no study to date has provided a calibration methodology designed to calculate human body structure (human body segments' length) using inertial sensors, which probably because no one to date has focused on this filed and considered this would be doable. In virtual space, human body size calibration is of significance to applying inertial sensors to track, recognize, and control motions. In virtual reality games context, if without the presence of an avatar, players are not able to see their real body when completely immersed in the virtual reality environment, plus their actual body structure size might be different from the virtual game settings, all of which would increase the complexity of learning game control. Furthermore, studies have shown that the virtual presence of an avatar could positively improve players' self-report of embodiment so to alter their response and actions in virtual space, and a calibrated size-matched avatar allows players to further enhance their abilities of perception and action judgment in virtual space [80], [81]. For instance, when trying to grabbing a chair in virtual space, players might find it easier and more accurate to perform this reaching action if shown a calibrated sized- 
matched avatar because they could directly compare their body parts length and positions to the objects in virtual space. Therefore, no matter with the virtual presence of an avatar or not, through applying this human body structure calibration methodology (proposed in this thesis) to calculate players' body segments length and customize the game character body size settings in the virtual reality motion game context, it may help to reduce the learning time required for players to adapt to game control, and improve the accuracy of game operation in virtual space.

\subsubsection{Constant Bias Calibration}

The constant bias for an accelerometer is typically referred to the offset of its output value from the true signal. To estimate the constant bias, it can leverage the basic principle that the static accelerometer should read gravitational acceleration [31], and place inertial sensor on a leveled surface with each axis of sensor alternatively pointing up and down in static condition, then respectively observe the accelerometer's output in each axis over a period of time [27].

\subsubsection{Alignment: Sensor-To-Body Calibration}

When collecting and analyzing data from inertial sensors, due to differences may exist among of the coordinates frame of each sensor, researchers should pay special attention to align the coordinates frames of different inertial sensors, and the coordinates frames of inertial sensors and body segments [61]. However, in a large number of the studies [53], [54], [55], [57], with the assumption that the axes of sensors and the axes of body segment where the sensor is attached are perfectly aligned, typically this alignment was neither included in the experiment process nor carried out by manually adjusting the inertial sensors placement. In fact, this alignment could be very difficult in practice, and one of the critical reasons is that sensors need to be placed on the curved body surface and have to avoid the main skeletal muscle groups, which could make it difficult to find sensor placement areas to perfectly align their coordinates frames [61].

To better perform the alignment of the coordinates frames of inertial sensors to improve accuracy and reliability, a sensor-to-body calibration procedure is needed [69], 
which has been named in different ways, such as functional calibration [58], anatomical calibration [70][71], system calibration [72], as well as sensor to segment calibration [73].

The sensor-to-body alignment methodology could be categorized as four types, including direct anatomical landmark identification (ALI), imposition of joint constraints (JCI), multibody modeling (MBM), and also functional approach (FUA) [61]. Detailed explanations are as follows:

Direct Anatomical Landmark Identification (ALI): It uses special devices (such as an IMU embedded caliper) to perform sensor-to-body calibration, with the device rotating around the axes of the human body frame and its ends pointing to the human body structure markers [71]. ALI's setup time is longer, and the required body segments need to be calibrated one by one [61].

Imposition of Joint Constraints (JCI): Applicable for arbitrary motions, it performs the sensor-to-body calibration leveraging the limited Degree of Freedom for joints, e.g., the knee and elbow [69].

Multibody Modelling (MBM): It can model human body structure through linking together multiple segments of the human body with rotational joints [74], and could perform sensor-to-body alignment through predefined motions or arbitrary movements [72], [75]. Inertial sensors are required to be placed on both ends of the each body segment with known distances, and would not work well when inertial sensors are only attached to the partial segments in a motion chain [61].

Functional Approach (FUA): It is the most popular technique for sensor-to-body calibration, and has been widely applied to estimate the joint angle for the upper and lower limbs [73], [76], [77], [78], [79], which requires the experiment participants to perform the predestinate movements and static postures [60] to calculate and align the coordinates frames of the sensor and the body segment through the angular velocity and the acceleration. However, the accuracy of FUA dramatically depends on the users' performing accuracy of the predefined gestures and movements [61].

Therefore, as stated above, calibration always possesses significance for applying inertial sensors, and is important for the performance and effectiveness of the inertial sensor-based motion tracking and control systems. In the past decades, numerous studies 
have focused on inertial sensors calibration to address the issues mentioned previously, such as deterministic errors, stochastic errors, and alignment issues. However, there does not exist a calibration methodology leveraging inertial sensors to calibrate human body structure to calculate body segments' length, which would have great potential to combine with the inertial sensor-based motion tracking and control system to improve its accuracy and usability in virtual reality game context. Based on this consideration, in this thesis, an inertial sensor-based human body structure calibration methodology will be proposed. 


\section{Chapter: Research Method}

\subsection{Experimental Equipment}

The sensor system used in this research is CC2650STK provided by Texas Instruments (http://www.ti.com/sensortag), which is compact and lightweight $(49.5 \mathrm{~mm} \times$ $37.5 \mathrm{~mm} \times 12.5 \mathrm{~mm}$ ), battery powered, and contains 10 low-power microelectromechanical (MEMs) sensors, including sensors for light, magnetic sensor, humidity, pressure, accelerometer, gyroscope, magnetometer, object temperature, ambient temperature, and digital microphone. CC2650STK is also embedded with Bluetooth transceiver, which can support wireless connection and data transmission from the sensor device to its accompanying PC or mobile data monitor platform. Figure 1 shows more detailed illustrations of the experimental equipment used in this research, including the outward appearance and the inside circuit board of CC2650STK, and its accompanying wireless data monitor mobile platform used for data reading.

In addition, as shown in Figure 1, CC2650STK is attached to a Velcro strap, which not only can flexibly adapt to different user body size to fasten the sensor on user body spots but also allows fast and easy setup for experimentation.

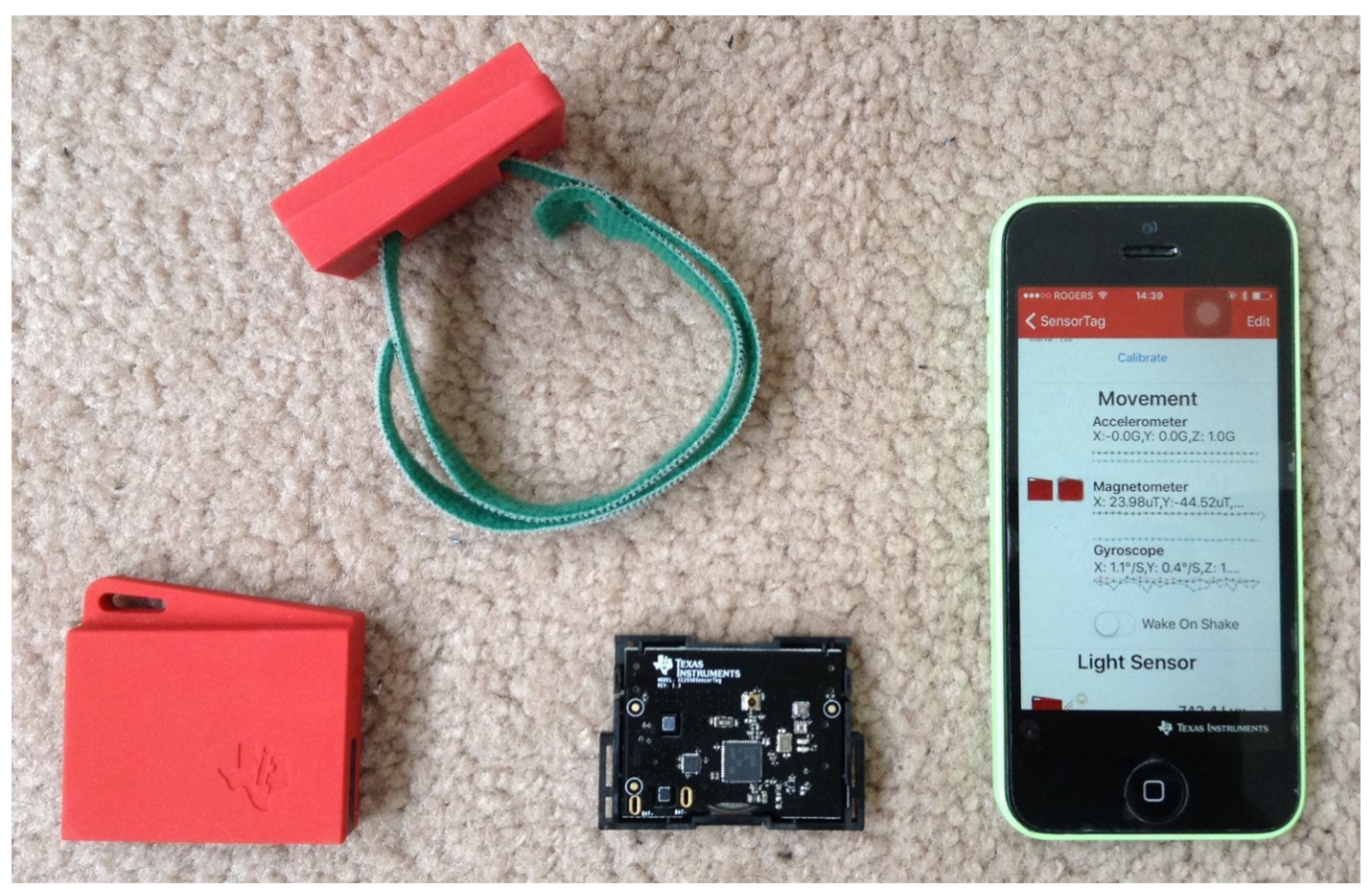

Figure 1. The outward appearance and the inside circuit board of Texas Instruments CC2650STK, and its accompanying wireless mobile data monitor platform. 
Furthermore, each sensor generates its own global reference frame upon setup. As shown in Figure 2, it illustrates the directions of the global X, Y, and Z axes generated by Texas Instruments CC2650STK used in this research, which shows that the positive Z-axis is oriented vertically downward towards the sensor device. Especially, this research relies on the acceleration sensor and tracks the accelerometer values in three perpendicular axes $\mathrm{X}, \mathrm{Y}$, and $\mathrm{Z}$. In static conditions, the accelerometer measures the gravitational acceleration value in each of the three axes.

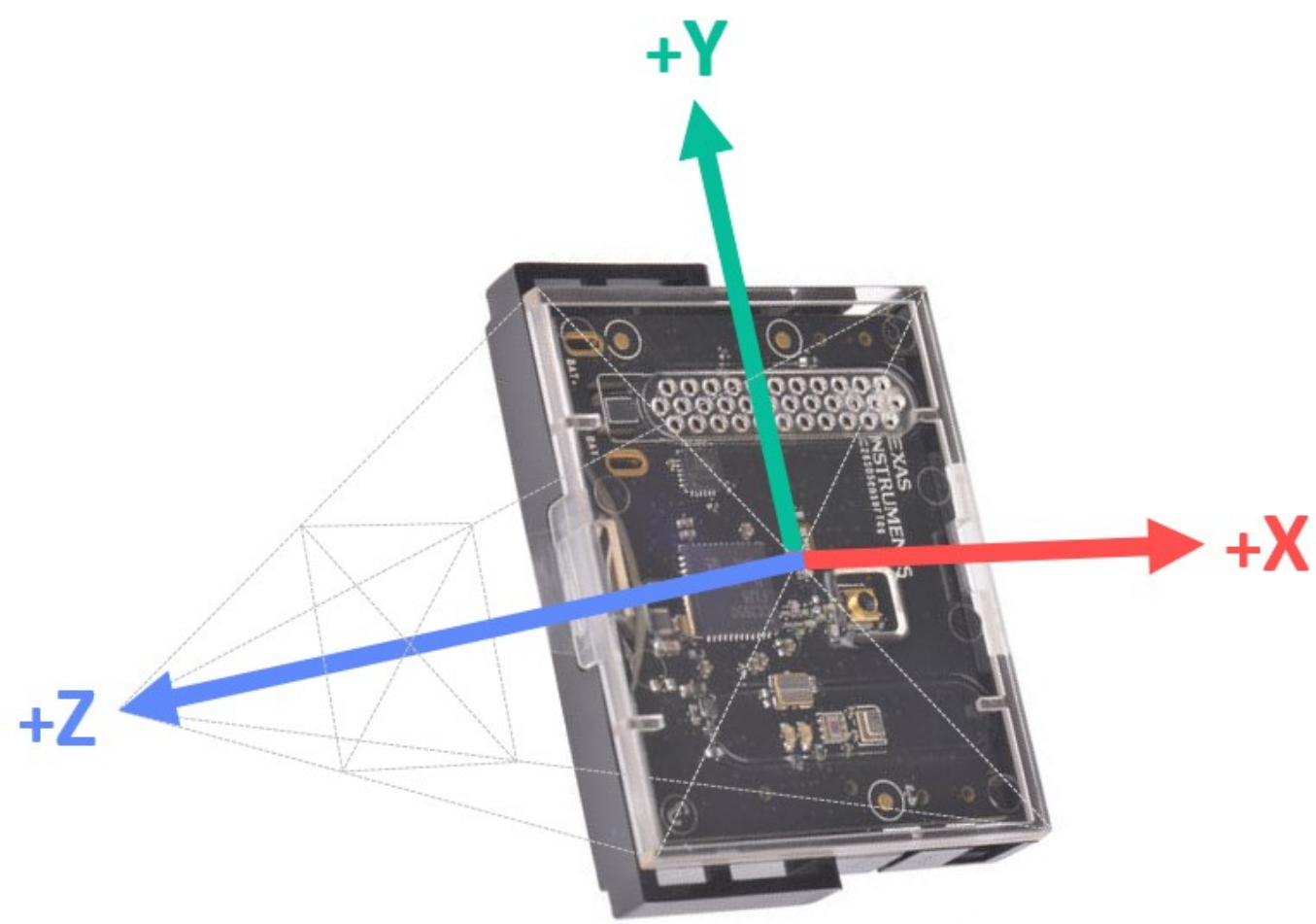

Figure 2. The directions of its global $X, Y$, and $Z$ axes generated by Texas Instruments CC2650STK used in this research. Global $X, Y$, and $Z$ axes are in color red, green and blue, respectively.

\subsection{Experimental Procedure}

Before providing details about this proposed calibration protocol, this section will provide an overview of the experimental procedure.

This proposed calibration methodology was tested on a total of 15 participants, whose ages range from 19 to 56 years old. This group of users was comprised of 10 males and 5 females, and they were all with normal physical conditions. The experiment sessions 
took place in a school, library, or user's home, which were familiar, convenient, and relaxing environments to participants. All experiment sessions were one-on-one based, which means only one participant was tested during each research session.

At the beginning of each test session, after a brief introduction of the background and general procedure of this research, an informed consent form was provided to each participant to read, and was collected after participants' signing, as required by Carleton University Research Ethics Board-B (CUREB-B).

Then the formal calibration procedure commenced. The entire calibration process in this research contained four static poses that participants must complete (explained in section 3.3). According to the different requirements of each pose, the required number of sensor devices would be two or four pieces, and researcher should verify the orientations of the sensor's global reference frame and the accuracy of the readings before getting started. As a preliminary step of each pose calibration, predefined numbers of sensors were fixed, as per the required orientations, to the designated body spots of participants using Velcro straps (for the specific body positions where sensors should be attached to for each pose calibration, please refer to section 3.3). Then each participant was provided with simple three-perspective simulating figure images (including front view, right view, and top view) to visually illustrate how to perform the poses, and also provided with accompanying printed and oral descriptions of key requirements to guide participants to properly perform the static poses. Next, the researcher checked and adjusted each participant's pose to guarantee it met the requirements and informed the participant to hold this pose still and allow some time for the researcher to record data. After achieving data stability, through taking the readings from the accompanying wireless data monitor mobile platform (with the numerical precision of one digit to the right of the decimal point), researcher manually recorded the $\mathrm{X}, \mathrm{Y}$, and $\mathrm{Z}$ axes accelerometer sensor values in the spreadsheet. Especially during data recording process, in the situations of stable and normal data fluctuation, the readings vibrating range was recorded (from the minimum to the maximum value), and the average value was used for later data analysis. Following the previously described test procedure, the four poses involved in this calibration protocol were tested successively. The completion of the four poses calibration marked the ending point of the calibration session, then the researcher measured and noted the total time 
duration for this calibration session. After all poses testing had done, aimed at verifying the accuracy of the calibration results (the calculated length of body parts) through comparison with the real length, the researcher used a ruler to manually measure the real length of participants' body segments, including the left and right sides of the whole leg (plus foot/shoe height), shinbone (plus foot/shoe height), forearm, upper arm, and torso, and recorded data in centimeters $(\mathrm{cm})$. At the end, the researcher expressed gratitude to participants with a token of a $\$ 5$ Tim Hortons gift card.

\subsection{Calibration Protocol}

\subsubsection{Calibration Protocol for Calculating Forearm Length (Pose 1)}

Figure 3 shows the three-perspective simulating figure to illustrate the calibration pose for calculating forearm length. The key points of this pose are listed as below:

- A total of two sensors is required for this calibration pose.

- Wearing sensors on left and right wrists with the same axes directions shown in Figure 3. Figure 3 shows the sensor axes directions on the right wrist. The sensor axes direction on the left wrist is a horizontal flip from the right wrist sensor direction, which means the left wrist sensor axes directions are: $+Y$-axis pointing to the back and $+\mathrm{X}$-axis pointing to the sky.

- Sitting down and putting both elbows on a horizontal table and holding both hands solidly together with the fingers of both hands crossing each other and both wrists solidly touching each other. Making the distance between both elbows equal to $30 \mathrm{~cm}$ (the middle points of both elbows serve as the starting point and ending point when measuring the distance between both elbows). Keeping poses of the left and right sides symmetrical to each other. Making both forearms perpendicular to the horizontal table to make $\mathrm{Y}$-axis values of the left and right accelerometer sensors read $\mathrm{Y}=0 \mathrm{G}$, then holding this arm position constant.

- Recording $\mathrm{X}$ and $\mathrm{Z}$ axes values of the left and right accelerometer sensors. 

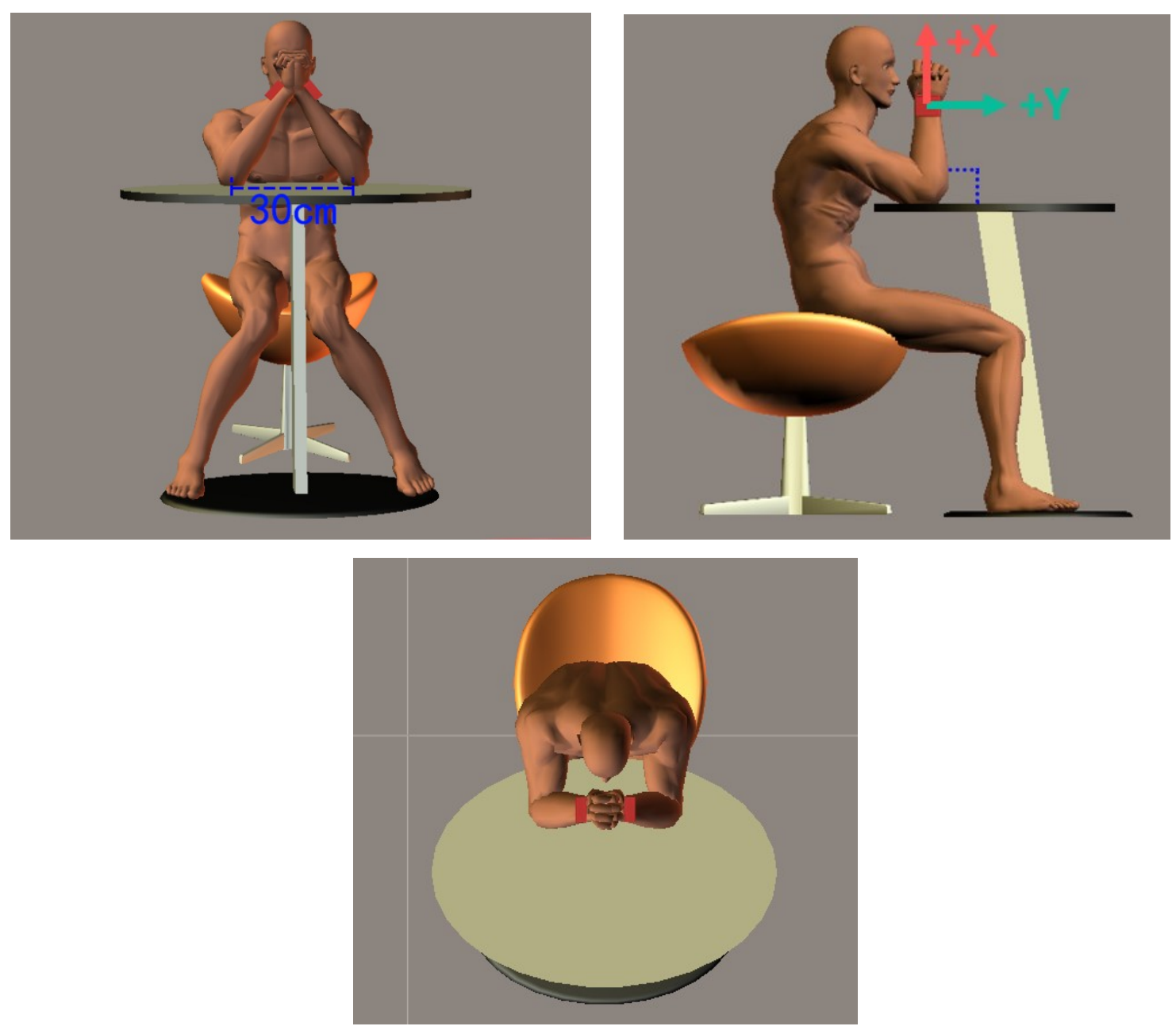

Figure 3. The three-perspective simulating figure to illustrate the calibration pose for calculating forearm length (from the front view, right view, and top view).

\subsubsection{Calibration Protocol for Calculating Shinbone Length (Plus Foot/Shoe}

\section{Height) (Pose 2)}

Figure 4 shows the three-perspective simulating figure to illustrate the calibration pose for calculating the shinbone length (plus foot/shoe height). The reason why "plus foot/shoe height" when calibrating the shinbone length is that this proposed calibration methodology calculates the length of the lower limbs, e.g., the whole leg and the shinbone, starting from the ground. The key points of this pose are listed as below:

- A total of two sensors is required for this calibration pose.

- Wearing sensors on lateral sides of both left and right ankles with the same axes directions shown in Figure 4. Figure 4 shows the sensor axes directions on the right ankle. The sensor axes direction on left ankle is a horizontal flip from right ankle 
sensor directions, which means left ankle sensor axes directions are: $+Y$-axis pointing to the front and $+\mathrm{X}$-axis pointing to the ground.

- Sitting down on a chair with both left and right shinbones separated from each other and both feet facing towards front, making the distance between both feet equal to $60 \mathrm{~cm}$ (the middle points of both feet serve as the starting point and ending point when measuring the distance between both feet), and putting both knees as close to each other as possible. Keeping poses of both left and right sides symmetrical to each other. Making both shinbones perpendicular to the horizontal ground to make $\mathrm{Y}$-axis values of the left and right accelerometer sensors read $\mathrm{Y}=0 \mathrm{G}$. Holding this leg position constant.

- Measuring and recording the distance between both knees in the unit of centimeters (the middle points of the left and right knees serve as the starting point and ending point when measuring the distance between both knees).

- Recording $\mathrm{X}$ and $\mathrm{Z}$ axes values of the left and right accelerometer sensors. 

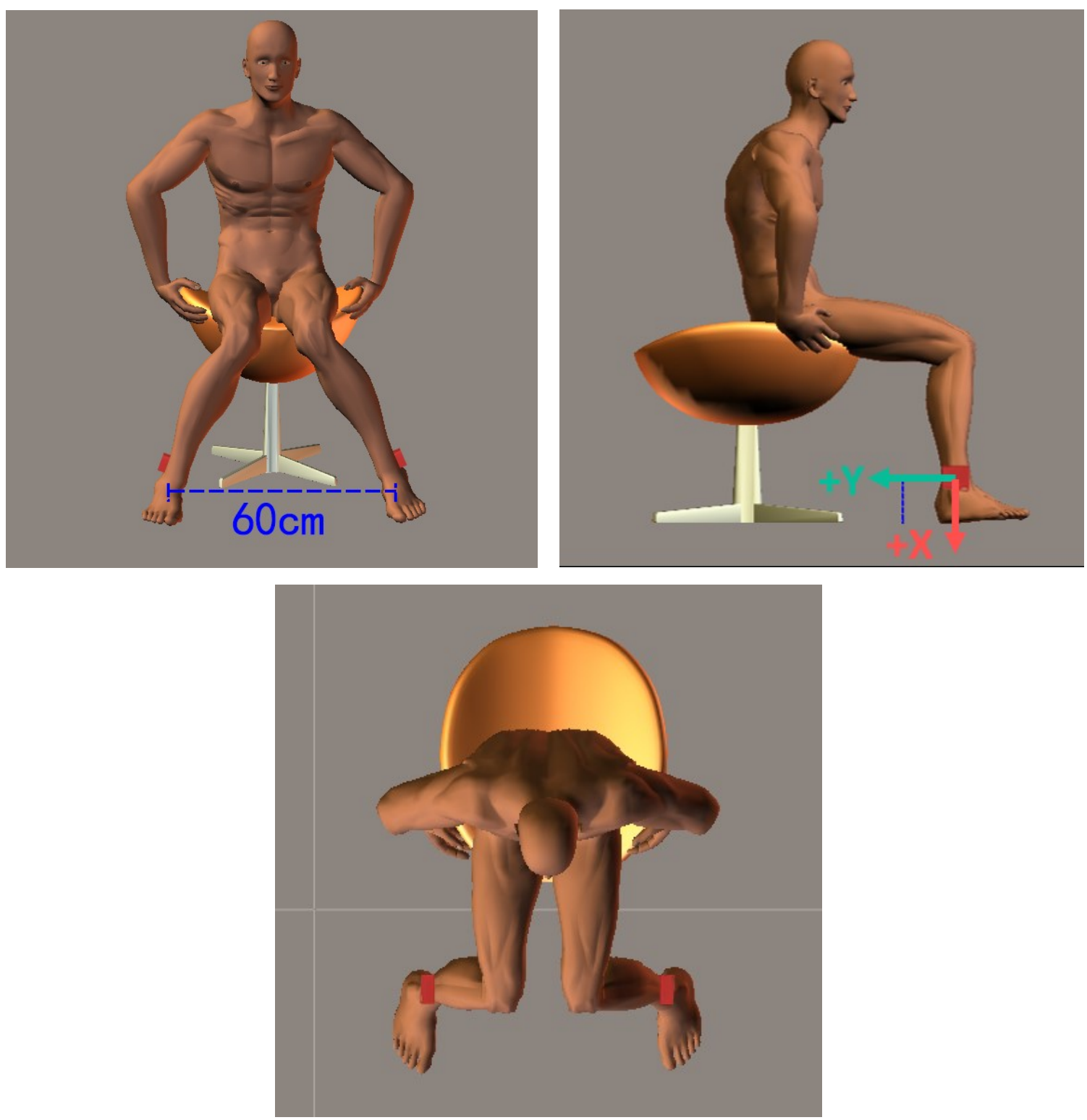

Figure 4. The three-perspective simulating figure to illustrate the calibration pose for calculating the shinbone length (plus foot/shoe height, from the front view, right view, and top view).

Originally, "knees can touch together" is another separate situation for calibrating shinbone length (plus foot/shoe height), and detailed explanations of this calibration protocol are shown in Appendix A.1. However, considering some users might have difficulties to touch both left and right knees together, we unified the situations of calibration shinbone length (plus foot/shoe height), so users can simply put their both knees as close to each other as possible to their comfortable extent. 


\subsubsection{Calibration Protocol for Calibrating the Whole Leg Length (Plus Foot/Shoe Height) (Pose 3)}

Figure 5 shows the three-perspective simulating figure to illustrate the calibration pose for calculating the whole leg length (plus foot/shoe height). The key points of this pose are listed as below:

- A total of two sensors is required for this calibration pose.

- Wearing sensors on lateral sides of both left and right knees with the same axes directions shown in Figure 5. Figure 5 shows the sensor axes directions on the right leg. The sensor axes direction on the left leg is a horizontal flip from the right leg sensor axes directions, which means the left leg sensor axes directions are: $+Y$-axis pointing to the front and $+\mathrm{X}$-axis pointing to the ground.

- Standing up with left and right legs separated from each other and both feet facing towards the front, making the distance between both feet equal to $60 \mathrm{~cm}$ (the middle points of both feet serve as the starting point and ending point when measuring the distance between both feet). Keeping poses of both left and right sides symmetrical to each other. Making both legs perpendicular to the horizontal ground to make Y-axis values of the left and right accelerometer sensors read $\mathrm{Y}=0 \mathrm{G}$, and hold this leg position constant.

- $\quad$ Recording $\mathrm{X}$ and $\mathrm{Z}$ axes values of the left and right accelerometer sensors. 

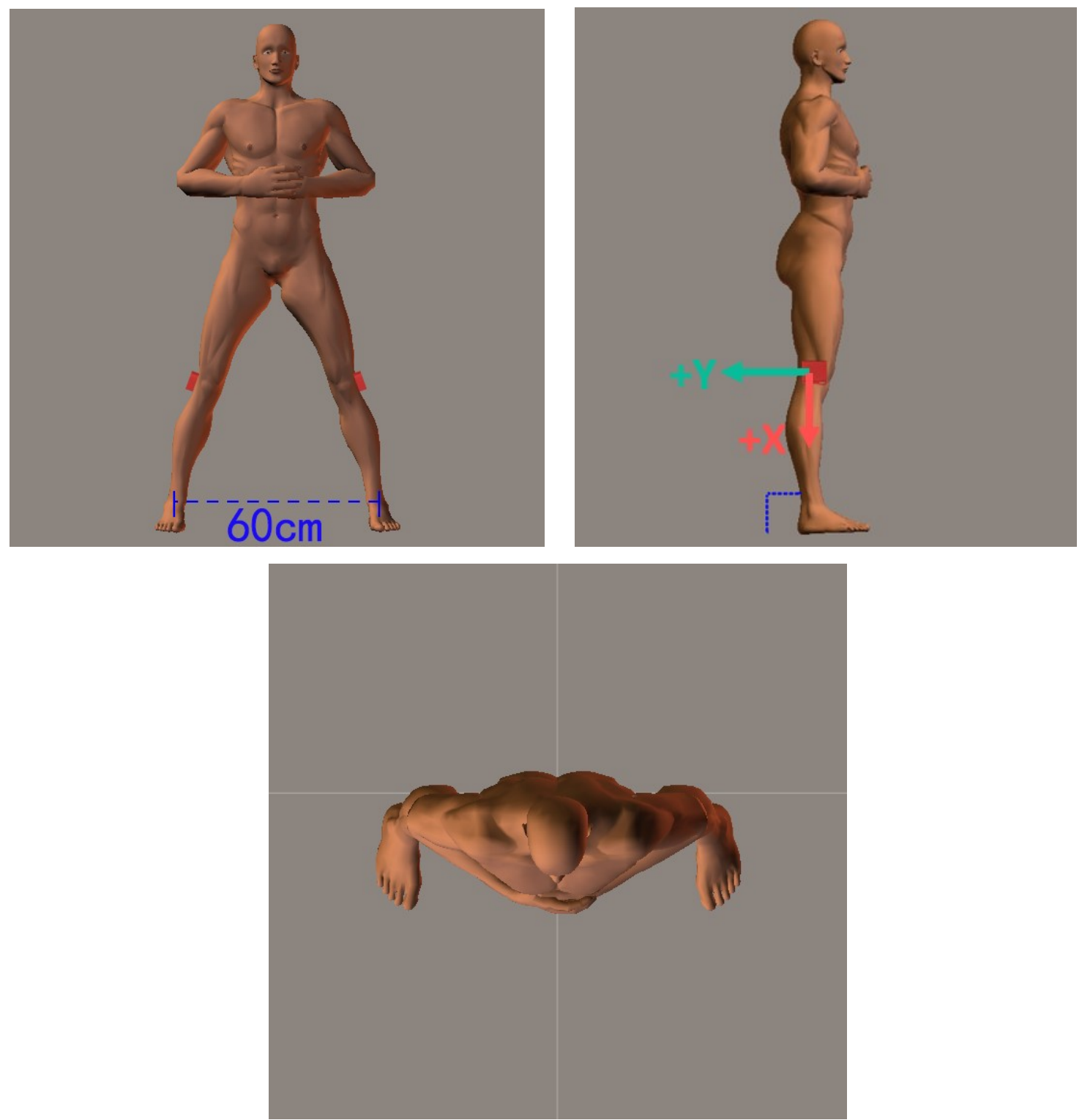

Figure 5. The three-perspective simulating figure to illustrate the calibration pose for calculating the whole leg length (plus foot/shoe height, from the front view, right view, and top view).

\subsubsection{Calibration Protocol for Calibration Upper Arm Length, and Torso Length}

\section{(Pose 4)}

Figure 6 shows the three-perspective simulating figure to illustrate the calibration pose for calculating the upper arm length and the torso length. The key points of this poses are listed as below:

- A total of four sensors is required for this calibration pose. 
- Wearing sensors on both left and right wrists as well as the middle points of both upper arms, with the same axes directions shown in Figure 6. Figure 6 shows the sensors axes directions on both the left and right sides.

- Standing straight, putting both hands on the pelvic bone edge, adjusting arms' position to make all of four sensors facing towards the front. Observing from the front perspective, wrists' sensors should touch the outline of the body (neither far away nor overlap inside). On each side of arms, keeping the upper arm and forearm into a straight line which should be perpendicular to the horizontal ground to make both Z-axis values of accelerometer sensors on the wrist and upper arm simultaneously read $Z=0 \mathrm{G}$. Holding this arm position constant.

- Recording X and Y axes values of all four accelerometer sensors. 

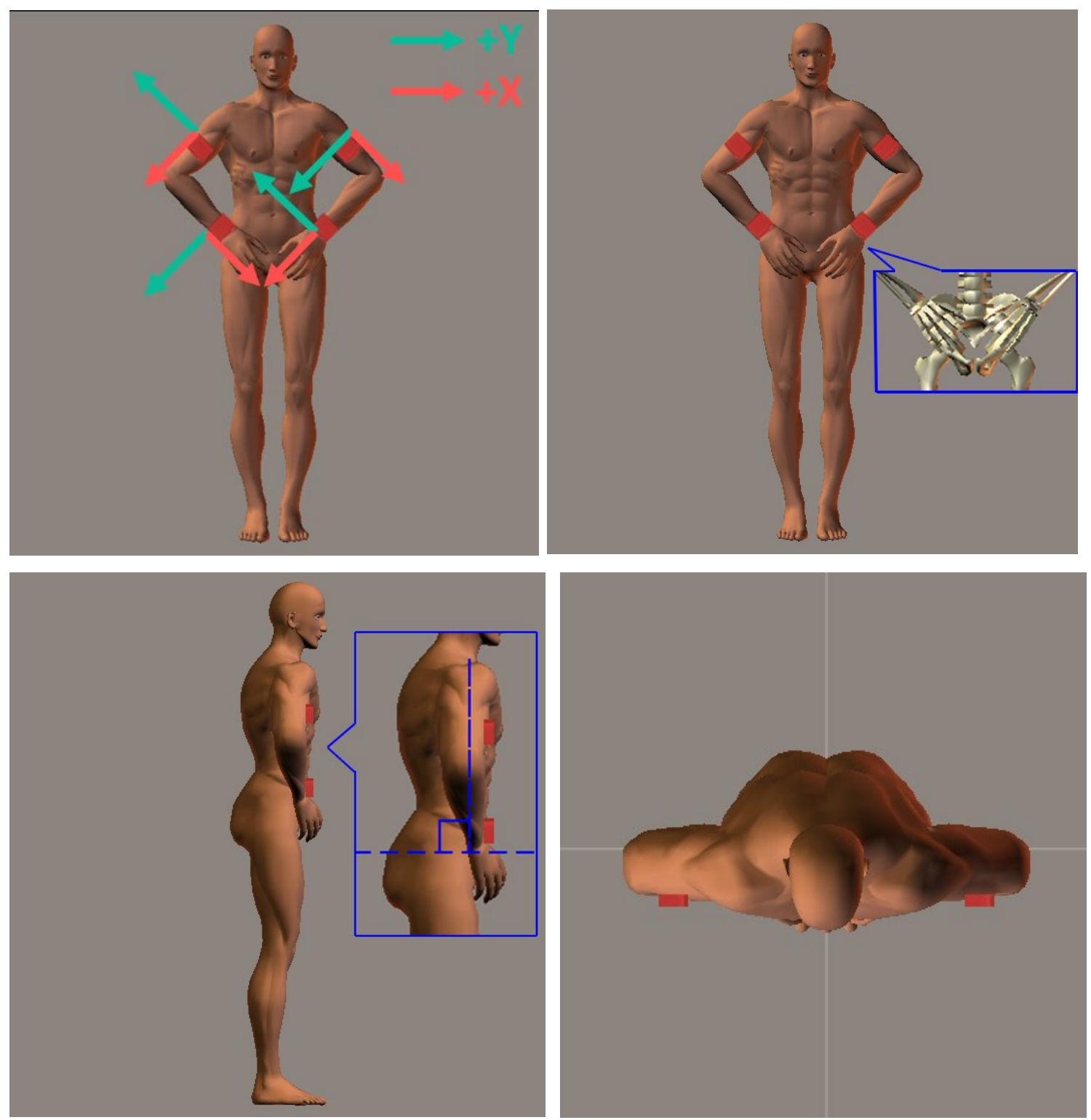

Figure 6. The three-perspective simulating figure to illustrate the calibration pose for calculating the upper arm length and the torso length (from the front view, right view, and top view).

\subsection{Calibration Algorithm}

\subsubsection{Calibration Algorithm for Forearm Length}

Based on the forearm length calibration protocol stated previously, to calculate the forearm length, the angle between the forearm and the horizontal table is needed to be calculated first. The calculation formulas and details are shown as below.

When $\mathrm{Y}$-axis value of accelerometer sensor reads $\mathrm{Y}=0 \mathrm{G}$, the angle between the forearm and the table is rotated about $\mathrm{Y}$-axis, so the angle is represented as roll $(\rho)$, and 
the $\mathrm{X}$ and $\mathrm{Z}$ axes values of accelerometer sensor are used to calculate the angle $\rho$. As shown in Figure 7, $\rho$ represents the angle between the forearm and the table, $\rho$ left represents the angle between the left forearm and the table, and $\rho_{\text {right }}$ represents the angle between the right forearm and the table; $\mathrm{X}$ represents the forearm length, $\mathrm{X}_{\text {left }}$ represents the left forearm length, and $X_{\text {right }}$ represents the right forearm length; accXval ${ }_{\text {left }}$ and acc $Z v a l_{\text {left }}$ respectively represent the $X$ and $Z$ axes values of the accelerometer sensors worn on the left wrist, and $\operatorname{accXval}_{\text {right }}$ and accZval ${ }_{\text {right }}$ respectively represent the $\mathrm{X}$ and $\mathrm{Z}$ axes values of the accelerometer sensors worn on the right wrist. Therefore, the calibration algorithms are shown as Equation (1) and (2):

The left forearm length calculation algorithm:

$$
\begin{aligned}
\rho_{\text {left }} & =\Pi+\operatorname{atan} 2\left(\operatorname{accXval}_{\text {left, }} \operatorname{accZval}_{\text {left }}\right) \\
& =\arctan \left(\operatorname{accXval}_{\text {left }} / \operatorname{accZval}_{\text {left }}\right) \\
\mathrm{X}_{\text {left }} & =15 \mathrm{~cm} / \cos \rho_{\text {left }}
\end{aligned}
$$

The right forearm length calculation algorithm:

$$
\begin{aligned}
\rho_{\text {right }} & =\Pi+\operatorname{atan} 2\left(\operatorname{accXval}_{\text {right }}, \operatorname{accZ}_{\text {val }}{ }_{\text {right }}\right) \\
& =\arctan \left(\operatorname{acc} X v a l_{\text {right }} / \operatorname{accZval}_{\text {right }}\right) \\
\mathrm{X}_{\text {right }} & =15 \mathrm{~cm} / \cos \rho \text { right }
\end{aligned}
$$




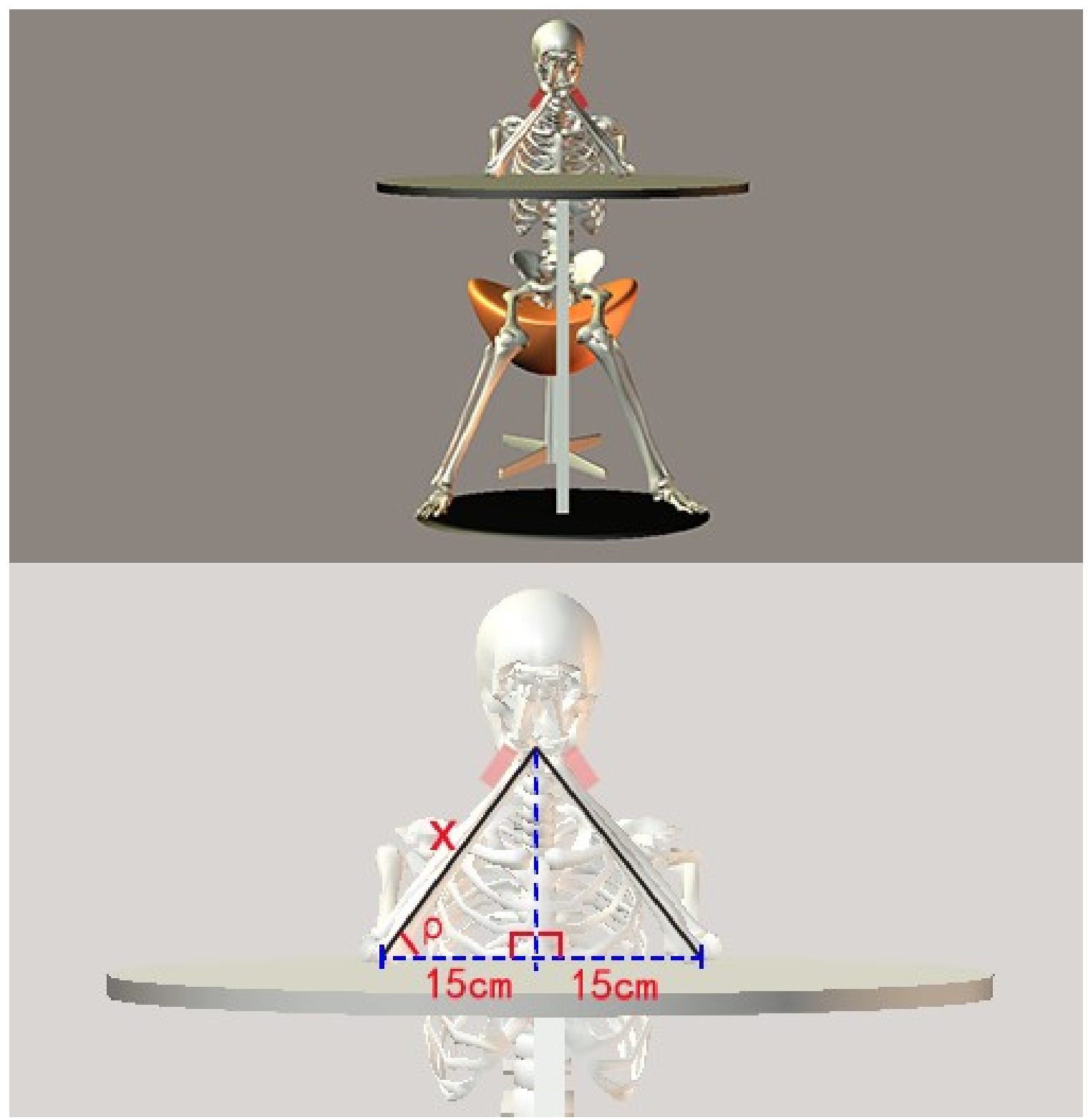

Figure 7. Skeletal model for explaining calibration algorithm for the forearm length, and for Pose 1 overview.

\subsubsection{Calibration Algorithm for Shinbone Length (Plus Foot/Shoe Height)}

Based on the shinbone length calibration protocol stated previously, to calculate the shinbone length (plus foot/shoe height), the angle between the shinbone and the ground is needed to be calculated first. The calculation formulas and details are shown as below.

Wearing sensors on the lateral sides of the left and right ankles, when performing this pose, the angle between the shinbone and the ground is rotated about $Y$-axis of sensors, so the angle is represented as roll $(\rho)$. When $\mathrm{Y}$-axis value of accelerometer sensor reads 
$\mathrm{Y}=0 \mathrm{G}$, the $\mathrm{X}$ and $\mathrm{Z}$ axes values of accelerometer sensor are used to calculate the angle $\rho$. As shown in Figure 8, $\rho$ represents the angle between the shinbone and the horizontal ground, $\rho_{\text {left }}$ represents the angle between the left shinbone and the ground, and $\rho_{\text {right }}$ represents the angle between the right shinbone and the ground; $\mathrm{X}$ represents the shinbone length (plus foot/shoe height), $\mathrm{X}_{\text {left }}$ represents the left shinbone length (plus foot/shoe height), and $\mathrm{X}_{\text {right }}$ represents the right shinbone length (plus foot/shoe height); $\mathrm{d}$ represents the distance between the left and right knees and unit is in centimeters (cm); AccXval left and accZval left respectively represent the $\mathrm{X}$ and $\mathrm{Z}$ axes values of the accelerometer sensor

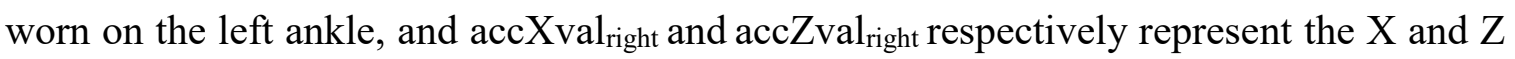
axes values of the accelerometer sensor worn on the right ankle. Therefore, the calibration algorithms are shown as Equation (3) and (4):

The left shinbone length (plus foot/shoe height) calculation algorithm:

$$
\begin{aligned}
\rho_{\text {left }} & =\Pi-\operatorname{atan} 2\left(\operatorname{accXval}_{\text {left, }}, \text { accZval }_{\text {left }}\right) \\
& =-\arctan \left(\left.\operatorname{accXval}\right|_{\text {left }} / \operatorname{accZval}_{\text {left }}\right) \\
\mathrm{X}_{\text {left }} & =[(30 \mathrm{~cm}-\mathrm{d} / 2) / 30 \mathrm{~cm}]^{*}\left[30 \mathrm{~cm} / \cos \rho_{\text {left }}\right]
\end{aligned}
$$

The right shinbone length (plus foot/shoe height) calculation algorithm:

$$
\begin{aligned}
\rho_{\text {right }} & =\Pi-\operatorname{atan} 2\left(\operatorname{accXval}_{\text {right }}, \text { accZval }_{\text {right }}\right) \\
& =-\arctan \left(\left.\operatorname{accXval}\right|_{\text {right }} / \operatorname{accZval} l_{\text {right }}\right) \\
X_{\text {right }} & =[(30 \mathrm{~cm}-\mathrm{d} / 2) / 30 \mathrm{~cm}]^{*}\left[30 \mathrm{~cm} / \cos \rho_{\text {right }}\right]
\end{aligned}
$$




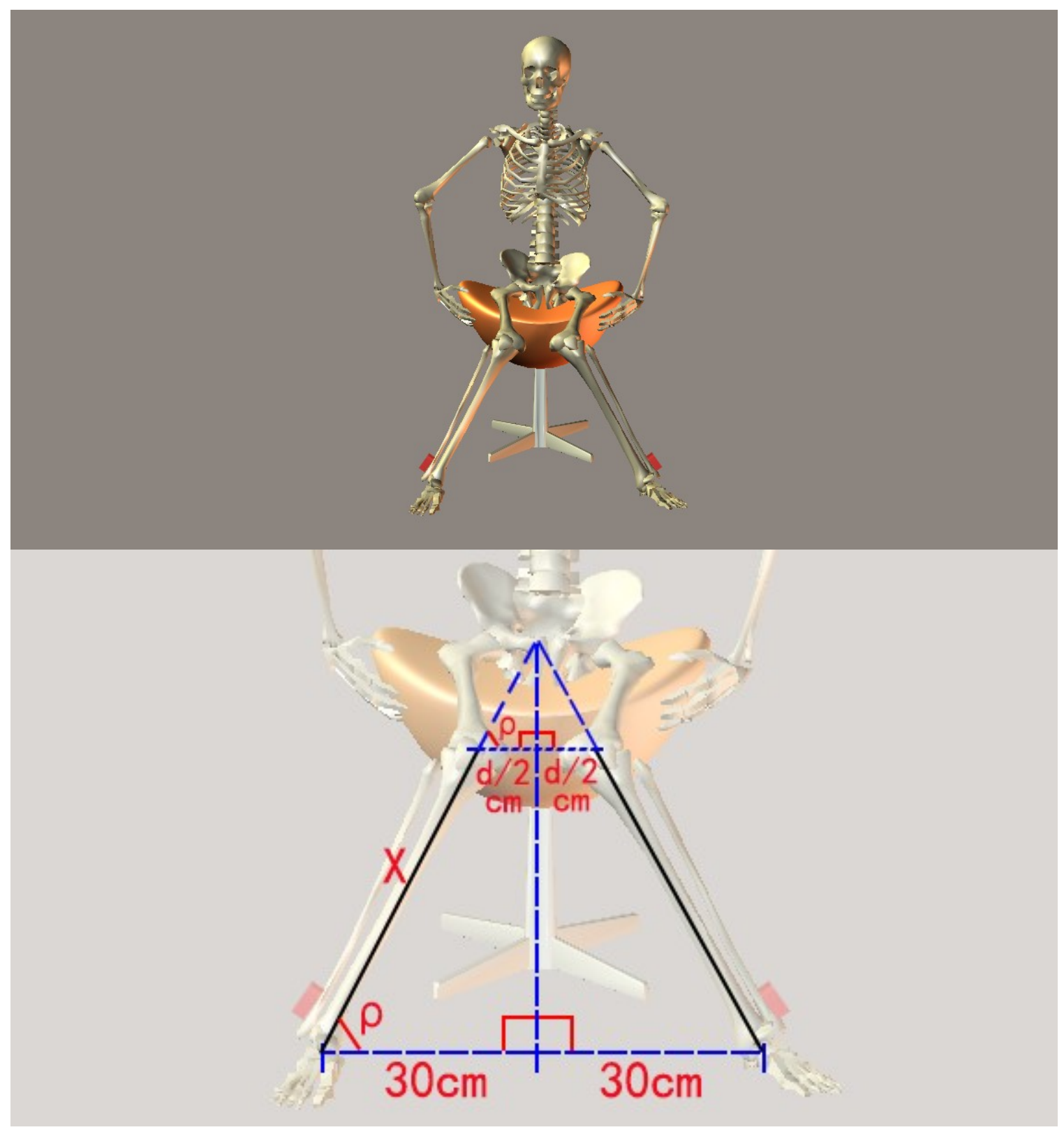

Figure 8. Skeletal model for explaining calibration algorithm for the shinbone length (plus foot/shoe height), and for Pose 2 overview.

Originally, "knees can touch together" is another separate situation for calibrating shinbone length (plus foot/shoe height), and detailed algorithm in this situation is shown in Appendix B.1. Considering some users might have difficulties to touch both knees together, we unified the two situations' algorithm, as shown in Appendix B.2. 


\subsubsection{Calibration Algorithm for Whole Leg Length (Plus Foot/Shoe Height)}

Based on the whole leg length calibration protocol stated previously, to calculate the whole leg length (plus foot/shoe height), the angle between the leg and the horizontal ground is needed to be calculated first. The calculation formulas and details are shown as below.

Wearing sensors on the lateral sides of the left and right knees, when performing this pose, the angle between the leg and the ground is rotated about Y-axis of sensors, so the angle is represented as roll $(\rho)$. When $\mathrm{Y}$-axis value of accelerometer sensor reads $\mathrm{Y}=0$ $\mathrm{G}$, the $\mathrm{X}$ and $\mathrm{Z}$ axes values of both accelerometer sensors are used to calculate the angle $\rho$. As shown in Figure 9, $\rho$ represents the angle between the leg and the ground, $\rho_{\text {left }}$ represents the angle between the left leg and the ground, and $\rho_{\text {right }}$ represents the angle between the right leg and the ground; $X$ represents the whole leg length (plus foot/shoe height), $X_{\text {left }}$ represents the left whole leg length (plus foot/shoe height), and $X_{\text {right }}$ represents the right whole leg length (plus foot/shoe height); AccXval ${ }_{\text {left }}$ and accZval ${ }_{\text {left }}$ respectively represent the $\mathrm{X}$ and $\mathrm{Z}$ axes values of the accelerometer sensor worn on the left knee, and accXval ${ }_{\text {right }}$ and accZval ${ }_{\text {right }}$ respectively represent the $\mathrm{X}$ and $\mathrm{Z}$ axes values of the accelerometer sensor worn on the right knee. Therefore, the calibration algorithms are shown as Equation (5) and (6):

The left whole leg length (plus foot/shoe height) calculation algorithm:

$$
\begin{aligned}
\rho_{\text {left }} & =\Pi-\operatorname{atan} 2\left(\operatorname{accXval}_{\text {left }}, \operatorname{accZval}_{\text {left }}\right) \\
& =-\arctan \left(\operatorname{accXval}_{\text {left }} / \operatorname{accZval}_{\text {left }}\right) \\
X_{\text {left }} & =30 \mathrm{~cm} / \cos \rho \rho_{\text {left }}
\end{aligned}
$$

The right whole leg length (plus foot/shoe height) calculation algorithm:

$$
\begin{aligned}
\rho_{\text {right }} & =\Pi-\operatorname{atan} 2\left(\operatorname{accXval}_{\text {right }}, \operatorname{accZval}_{\text {right }}\right) \\
& =-\arctan \left(\operatorname{acc} X v a l_{\text {right }} / \operatorname{accZval} l_{\text {right }}\right) \\
X_{\text {right }} & =30 \mathrm{~cm} / \cos \rho \text { right }
\end{aligned}
$$




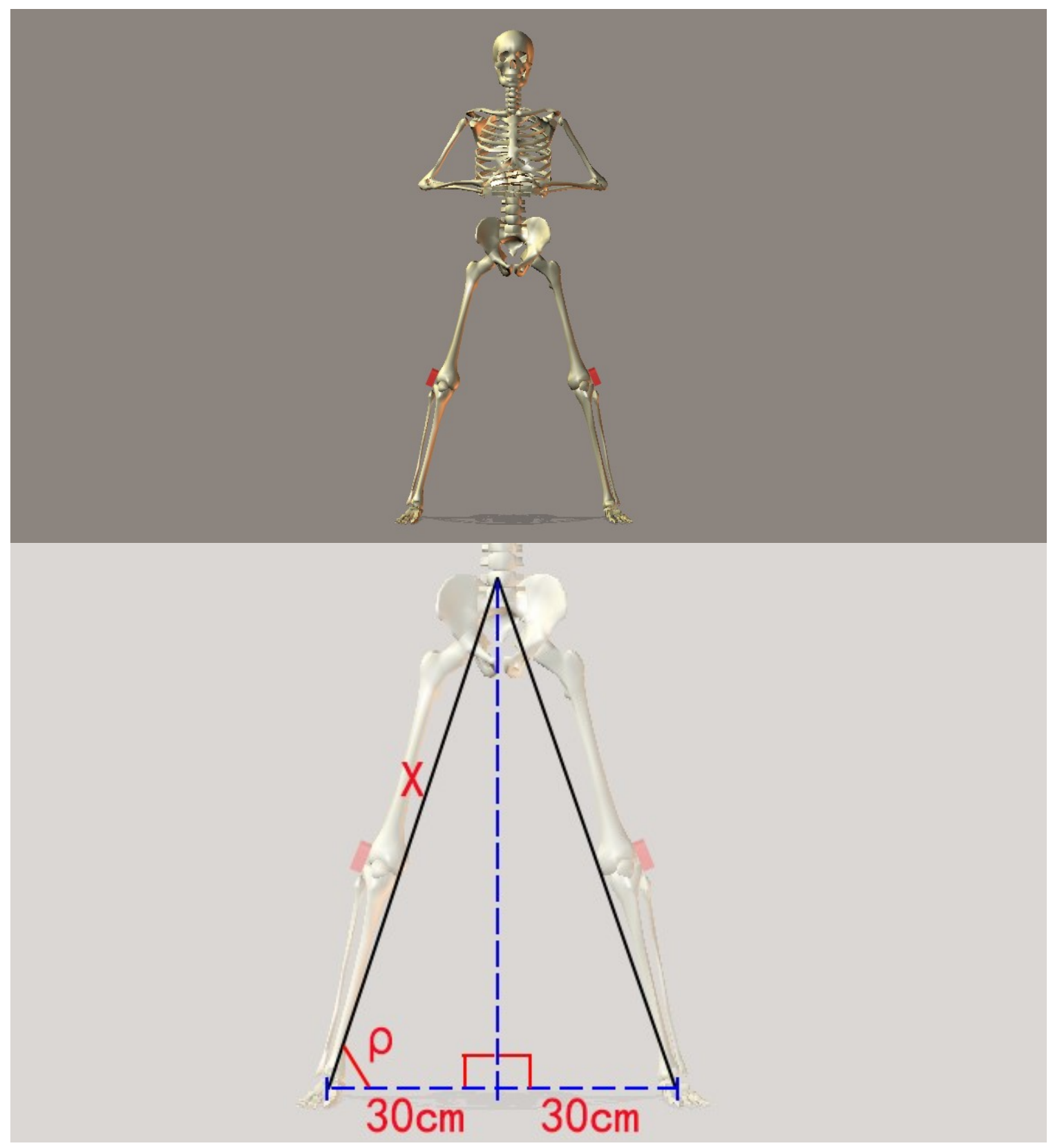

Figure 9. Skeletal model for explaining calibration algorithm for the whole leg length (plus foot/shoe height), and for Pose 3 overview.

\subsubsection{Calibration Algorithm for Upper Arm Length and Torso Length}

Based on the upper arm length calibration protocol stated previously, to calibrate the upper arm length, it leverages the already calculated forearm length (for details, please refer to section 3.4.1), and the angle between the forearm and upper arm. The calculation formulas and details are shown as below. 
For each side of arms, wearing sensors on the wrist as well as the middle point of the upper arm, when performing this pose, all sensors are facing towards the front, and the angle between the forearm and the upper arm is rotated about Z-axis of sensors, so the angle is represented as Yaw ( a ). For each side of arms, when adjusting the arm position to achieve both Z-axis values of accelerometer sensors on the wrist and the upper arm simultaneously read $\mathrm{Z}=0 \mathrm{G}$, the $\mathrm{X}$ and $\mathrm{Y}$ axes values of both accelerometer sensors on the wrist and the upper arm are used to calculate the angle a. As shown in Figure 10, making $a_{1 \text { left }}$ represents the angle between the left forearm and the horizontal ground, and $a_{2 \text { left }}$ represents the angle between the left upper arm and the ground; $a_{1 \text { right }}$ represents the angle between the right forearm and the ground, and $a_{2}$ right represents the angle between the right upper arm and the ground; $b$ is shown in Figure 10 and unit is centimeters $(\mathrm{cm}), b_{\text {left }}$ represents $\mathrm{b}$ on the left body side, $\mathrm{b}_{\text {right }}$ represents $\mathrm{b}$ on the right body side; $\mathrm{C}$ represents the already calculated forearm length in previous calibration step and unit is centimeters $(\mathrm{cm})$, $\mathrm{c}_{\text {left }}$ represents the already calculated left forearm length, $\mathrm{c}_{\text {right }}$ represents the already calculated right forearm length (for details, please refer to section 3.4.1); X represents the upper arm length, $X_{\text {left }}$ represents the left upper arm length, $X_{\text {right }}$ represents the right upper arm length. Therefore, the calibration algorithms are shown as Equation (7) and (8):

The left upper arm length calculation algorithm:

$$
\begin{aligned}
& a_{1 \text { left }}=\Pi-\operatorname{atan} 2\left(\operatorname{accXval}_{1 \text { left, }}, \text { accYval }_{1 \text { left }}\right) \\
& =-\arctan \left(\operatorname{accXval}_{1 \text { left }} / \operatorname{accYval}_{1 \text { left }}\right) \\
& \mathrm{b}_{\text {left }}=\mathrm{c}_{\text {left }} * \cos \mathrm{a}_{1 \text { left }} \\
& a_{2} \text { left }=\operatorname{atan} 2\left(\operatorname{accXval}_{2} \text { left, } \operatorname{accYval}_{2} \text { left }\right) \\
& =\arctan \left(\operatorname{accXval}_{2} \text { left } / \operatorname{accYval}_{2} \text { left }\right) \\
& \mathrm{X}_{\text {left }}=\mathrm{b}_{\text {left }} / \cos \mathrm{a}_{2} \text { left }
\end{aligned}
$$

The right upper arm length calculation algorithm:

$$
\begin{aligned}
& a_{1 \text { right }}=\operatorname{atan} 2\left(\operatorname{accXval}_{1} \text { right, } \operatorname{accYval}_{1 \text { right }}\right) \\
& =\arctan \left(\operatorname{accXval}_{1 \text { right }} / \mathrm{accYval}_{1 \text { right }}\right) \\
& \mathrm{b}_{\text {right }}=\mathrm{c}_{\text {right }} * \cos \alpha_{1 \text { right }} \\
& a_{2} \text { right }=\Pi-\operatorname{atan} 2\left(\operatorname{accXval}_{2} \text { right, } \operatorname{accYval}_{2} \text { right }\right) \\
& =-\arctan \left(\operatorname{accXval}_{2 \text { right }} / \mathrm{accYval}_{2 \text { right }}\right) \\
& \mathrm{X}_{\text {right }}=\mathrm{b}_{\text {right }} / \cos \alpha_{2 \text { right }}
\end{aligned}
$$


For the purpose of calibrating the torso length, as shown in Figure 10, making $\mathrm{Y}$ represents the torso length, and $Y=Y_{1}+Y_{2}$. $Y_{1}$ and $Y_{2}$ are shown in Figure 10, and unit is in centimeters (cm). The calibration algorithms are shown as Equation (9) and (10):

The torso length calculation algorithm (from left side values):

$$
\begin{aligned}
\mathrm{Y}_{1 \text { left }} & =\mathrm{c}_{\text {left }} * \sin \mathrm{a}_{1 \text { left }} \\
\mathrm{Y}_{2 \text { left }} & =\mathrm{X}_{\text {left }} * \sin \mathrm{a}_{2 \text { left }} \\
\mathrm{Y} & =\mathrm{Y}_{1 \text { left }}+\mathrm{Y}_{2 \text { left }}
\end{aligned}
$$

The torso length calculation algorithm (from right side values):

$$
\begin{aligned}
Y_{1 \text { right }} & =c_{\text {right }} * \sin a_{1 \text { right }} \\
Y_{2 \text { right }} & =X_{\text {right }} * \sin \alpha_{2} \text { right } \\
Y & =Y_{1 \text { right }}+Y_{2 \text { right }}
\end{aligned}
$$




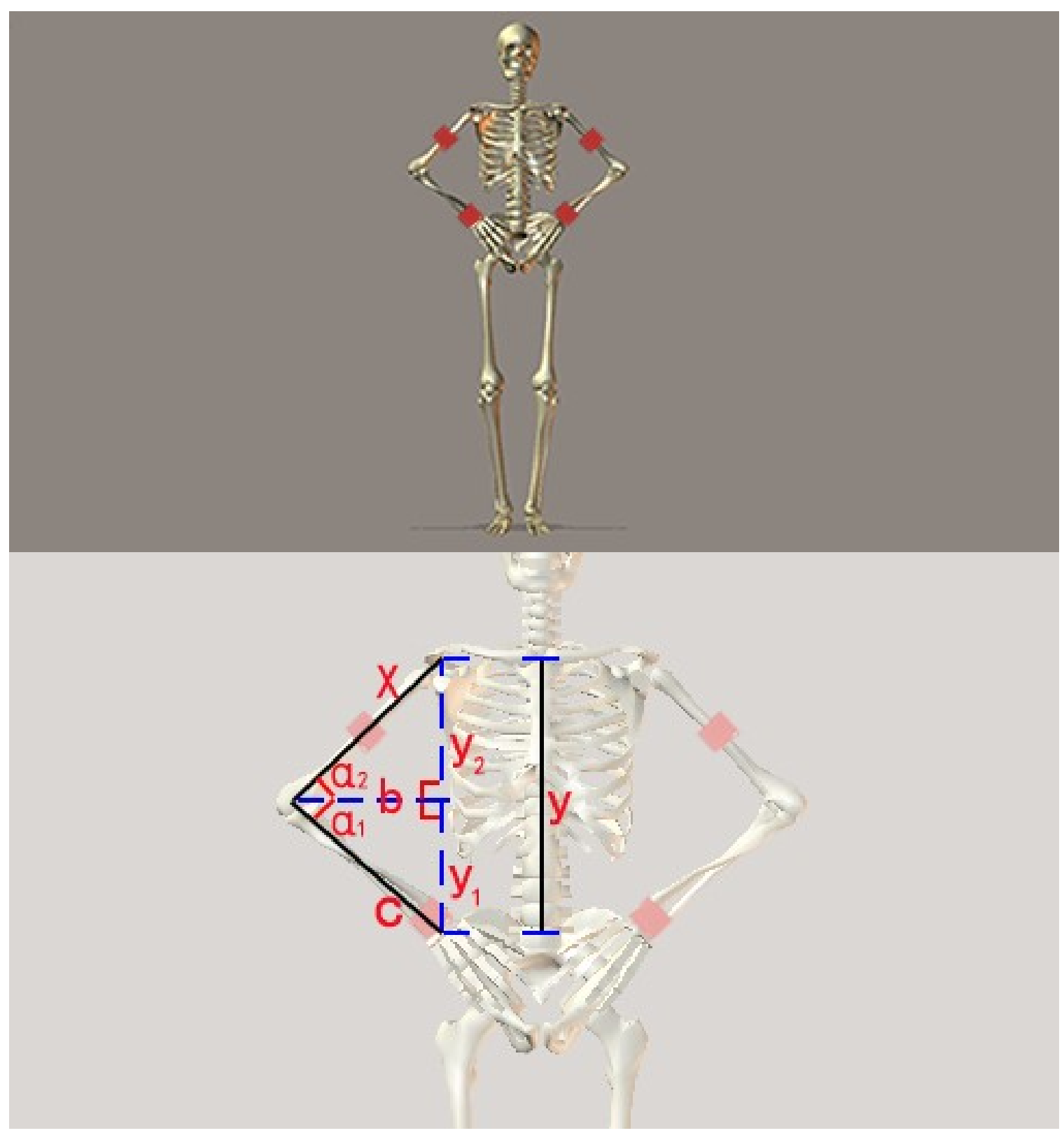

Figure 10. Skeletal model for explaining calibration algorithm for the upper arm length and the torso length, and for Pose 4 overview. 


\section{Chapter: Results Analysis}

This chapter will present the experiment results to understand the utility, advantages, and disadvantages of this proposed calibration methodology, including the average calibration accuracy across all participants, calibration accuracy for different body parts and individual participants, the comparison between calibration accuracy for the left and right human body sides, and calibration time duration.

The calibration accuracy mentioned in this chapter is measured by calibration error rate (\%), which is calculated by the following algorithm, as shown in Equation (11):

Calibration error rate $(\%)=$

( | body part real length - body part calibrated length | / body part real length) *100\%

In the above equation, the "body part calibrated length" is calculated based on the algorithm presented in section 3.4, and the "body part real length" is collected through ruler measurements during the experiment.

\subsection{Average Calibration Accuracy}

According to the experiment results, this proposed calibration system provides satisfactory calibration accuracy with overall $7.64 \%$ average error rate across all body parts and all participants.

Figure 11 shows the average calibration accuracy for different body parts, which reflects the average values of both body sides across all participants. As shown in Figure 11, in this experiment, this proposed calibration protocol provides relatively the best performance in calibrating the forearm, with a $4.88 \%$ average error rate, followed by the shinbone (plus foot/shoe height) with a $6.26 \%$ average error rate, whole leg (plus foot/shoe height) $7.24 \%$, torso $7.92 \%$, and upper arm with $11.82 \%$ average error rate, which is relatively worse than other body parts calibration results, but is still within the acceptable range. 


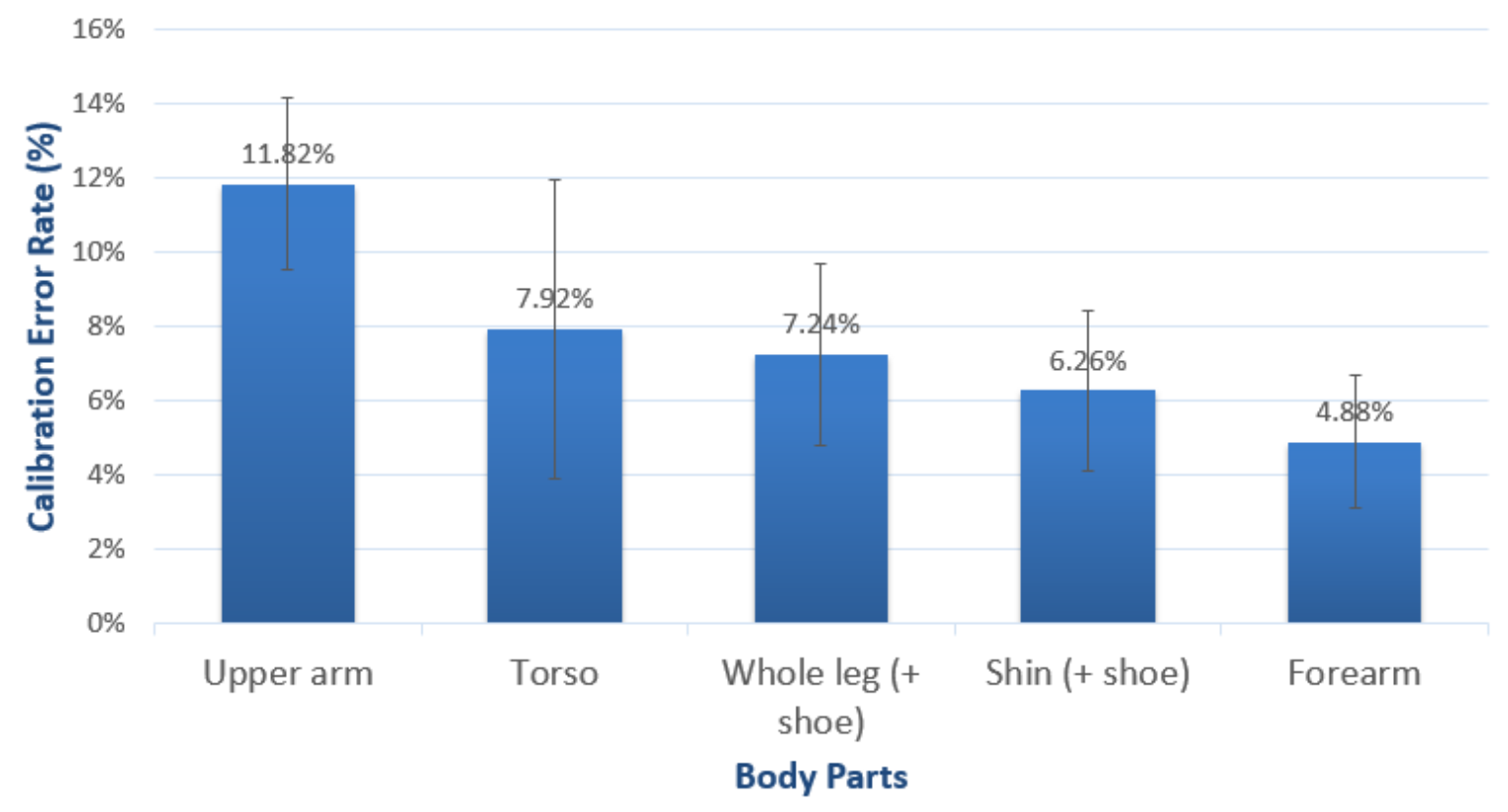

Figure 11. Calibration average error rates (\%) for different body parts. Calibration error rates (\%) in this chart reflect the average values for both body sides across all participants.

In addition, the individual participant's calibration accuracy for different body parts is shown in Figure 12, which is corresponding to the trends illustrated in Figure 11, revealing that, for each participant in this experiment, this proposed calibration methodology works best for forearm calibration, and upper arm calibrations generate relatively more errors when compared to the other body parts' calibration results.

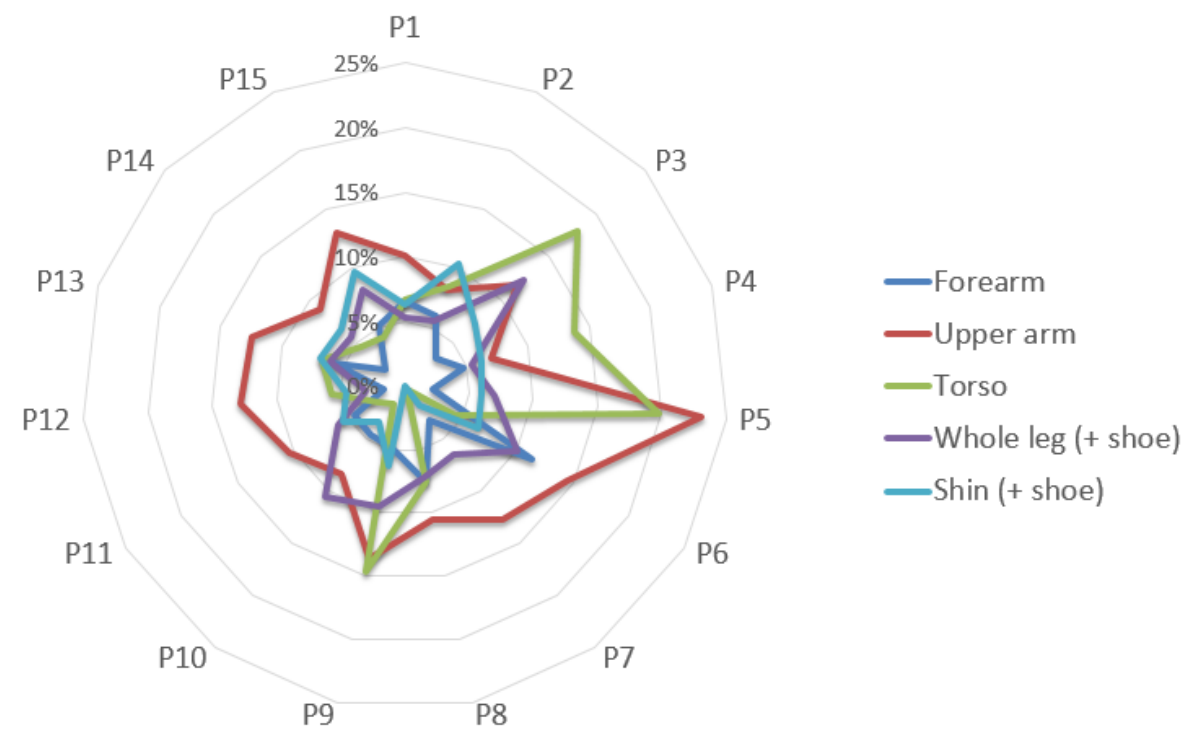

Figure 12. Individual participant's calibration error rates (\%) for different body parts (15 participants included), reflecting the average values for both body sides. 
These results are in line with expectations. The calibration poses for the forearm, the shinbone, and the whole leg are comparatively easier for participants to perform well when comparing to the pose for forearm and torso. Especially, the calibration methodology for the forearm is with relatively the easiest and most straightforward requirements for participants to meet, and also is the easiest for the researcher to ensure the sensor axis direction aligned well with participant's forearm bone direction, therefore it is not surprising to see that forearm achieves the best calibration accuracy among the overall calibration results in this experiment. By contrast, the calibration methodology for upper arm and torso is with more requirements and is relatively more complicated for participants. In addition, the calibration formulas for calculating the upper arm length leverages the calibrated forearm length in the previous step, hence the upper arm and torso calibration errors would also contain the errors generated from the forearm calibration. Therefore, due to the comparably more complicated calibration requirements and the cumulative errors, the upper arms calibration accuracy is affected to some extent, but is still acceptable.

\subsection{Comparison of Calibration Accuracy between Left and Right Body Sides}

The structure of the human body may not necessarily be completely symmetrical, e.g., the left and right forearms may not be exactly the same length. In addition, simultaneously calibrating the left and right body parts will require multiple sensor devices attached on both sides of the human body. Although the sensor devices are all the identical model, each sensor is unique and can possibly generate slightly different readings from other sensors even in the exactly same condition, which may lead to different calibration results when comparing calibration accuracy on the left and right body sides. Therefore, it appears necessary to simultaneously apply the calibration methodology on both the left and right body sides to verify and compare its calibration accuracy and stability.

\subsubsection{Forearm Length Calibration}

As shown in Figure 13, when simultaneously calibrating the left and right forearms, both sides provide outstanding calibration accuracy, with a $6.29 \%$ average calibration error rate for the left forearm, and $3.47 \%$ average calibration error rate for the right forearm. This 
shows that the proposed calibration methodology remains stable and robust, and forearm length calibration accuracy on both sides varies within the reasonable range, even if participants' bodies may not be perfectly symmetrical and slight variations may exist between the left and right sensors. This trend has been confirmed by the comparison between individual participants' calibration accuracy on the left and right body sides, as shown in Figure 14. This trend also has been detailed, explained, and examined through exploring the calibrated forearm length and the real forearm length on the left and right body sides, as shown in Table 1 and Table 2.

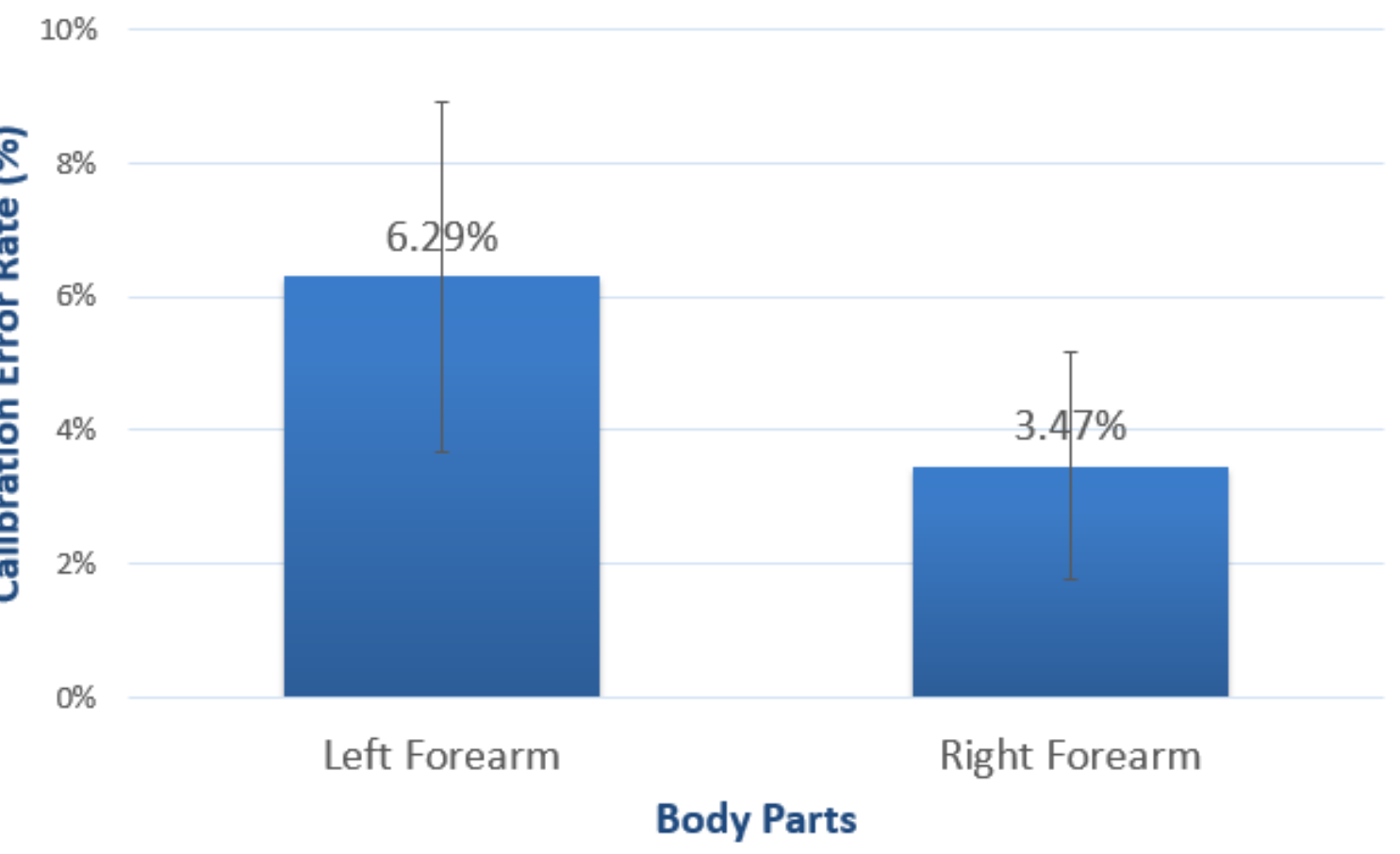

Figure 13. Forearm length calibration average error rates (\%) for the left and right body sides across all participants. 


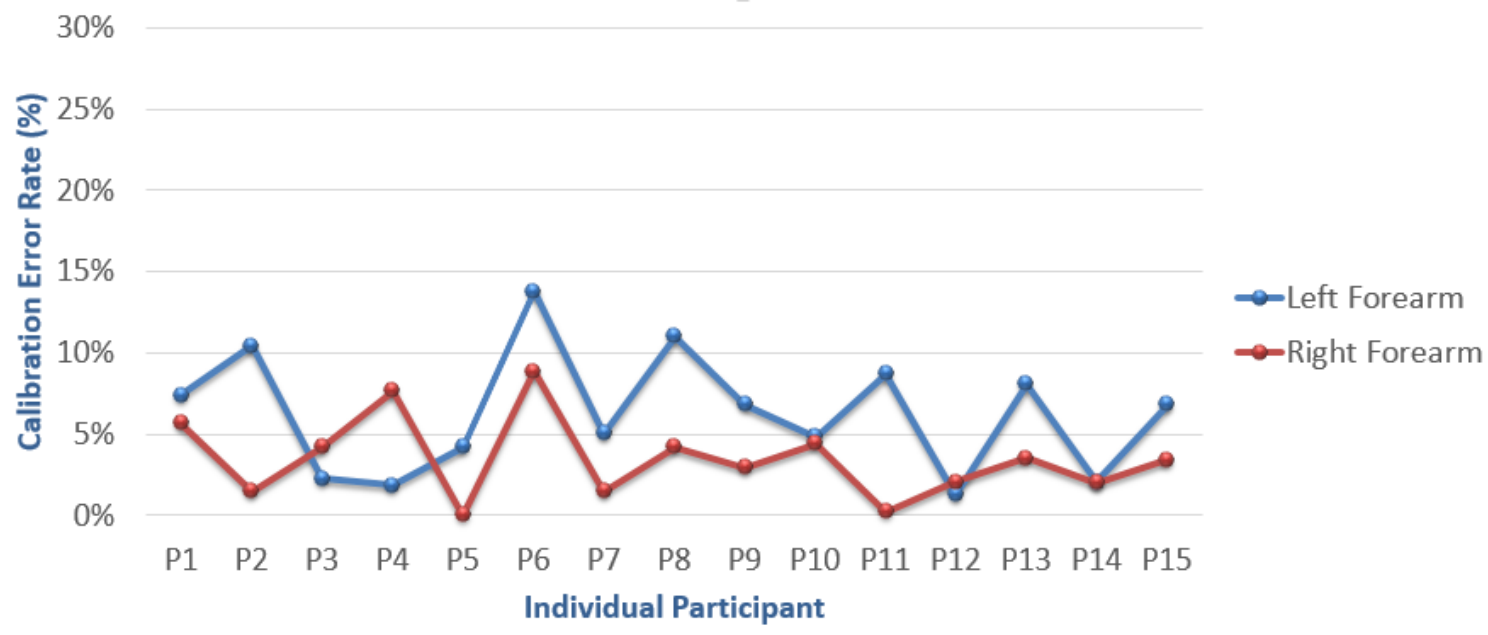

Figure 14. Forearm length calibration error rates (\%) on the left and right body sides for individual participants.

Table 1. Left forearm length calibration detailed data for individual participants, including calculated length $(\mathrm{cm})$, real length $(\mathrm{cm})$, and error rates $(\%)$.

\begin{tabular}{|l|c|c|c|c|c|c|c|c|}
\hline \multicolumn{9}{|c|}{ Left Forearm } \\
\hline Participant & P1 & P2 & P3 & P4 & P5 & P6 & P7 & P8 \\
\hline Error Rate (\%) & $7.41 \%$ & $10.45 \%$ & $2.26 \%$ & $1.83 \%$ & $4.19 \%$ & $13.79 \%$ & $5.03 \%$ & $10.99 \%$ \\
\hline Calculated length (cm) & 25.0 & 27.6 & 27.6 & 26.5 & 27.6 & 29.6 & 24.7 & 28.3 \\
\hline Real length (cm) & 27.0 & 25.0 & 27.0 & 26.0 & 26.5 & 26.0 & 26.0 & 25.5 \\
\hline Participant & P9 & P10 & P11 & P12 & P13 & P14 & P15 & \\
\hline Error Rate (\%) & $6.80 \%$ & $4.82 \%$ & $8.70 \%$ & $1.24 \%$ & $8.07 \%$ & $2.04 \%$ & $6.80 \%$ & \multirow{2}{*}{$/$} \\
\hline Calculated length (cm) & 28.3 & 28.3 & 25.0 & 22.8 & 26.5 & 25.0 & 28.3 & \\
\hline Real length (cm) & 26.5 & 27.0 & 23.0 & 22.5 & 24.5 & 24.5 & 26.5 & \\
\hline
\end{tabular}

Table 2. Right forearm length calibration detailed data for individual participants, including calculated length $(\mathrm{cm})$, real length $(\mathrm{cm})$, and error rates $(\%)$.

\begin{tabular}{|l|c|c|c|c|c|c|c|c|}
\hline \multicolumn{10}{|c|}{ Right Forearm } \\
\hline Participant & P1 & P2 & P3 & P4 & P5 & P6 & P7 & P8 \\
\hline Error Rate (\%) & $5.66 \%$ & $1.46 \%$ & $4.19 \%$ & $7.65 \%$ & $0.00 \%$ & $8.85 \%$ & $1.46 \%$ & $4.19 \%$ \\
\hline Calculated length (cm) & 25.0 & 25.4 & 27.6 & 24.0 & 25.0 & 28.3 & 25.4 & 27.6 \\
\hline Real length (cm) & 26.5 & 25.0 & 26.5 & 26.0 & 25.0 & 26.0 & 25.0 & 26.5 \\
\hline Participant & P9 & P10 & P11 & P12 & P13 & P14 & P15 & \\
\hline Error Rate (\%) & $2.92 \%$ & $4.42 \%$ & $0.21 \%$ & $2.03 \%$ & $3.53 \%$ & $1.99 \%$ & $3.42 \%$ \\
\hline Calculated length (cm) & 28.3 & 25.8 & 23.0 & 22.0 & 25.4 & 24.0 & 27.0 \\
\hline Real length (cm) & 27.5 & 27.0 & 23.0 & 22.5 & 24.5 & 24.5 & 28.0 & \\
\hline
\end{tabular}

\subsubsection{Upper Arm Length Calibration}

As shown in Figure 15, the average calibration accuracy for the left and right upper arm are similar to each other, showing the stable performance of the upper arm length 
calibration protocol. In addition, the upper arm's calibration accuracy still maintains a comparatively higher error rate (but still acceptable) when compared to the forearm length calibration accuracy on the left and right body sides, which is consistent with the findings in section 4.1. Figure 16, Table 3, and Table 4 also support and explain these similar trends.

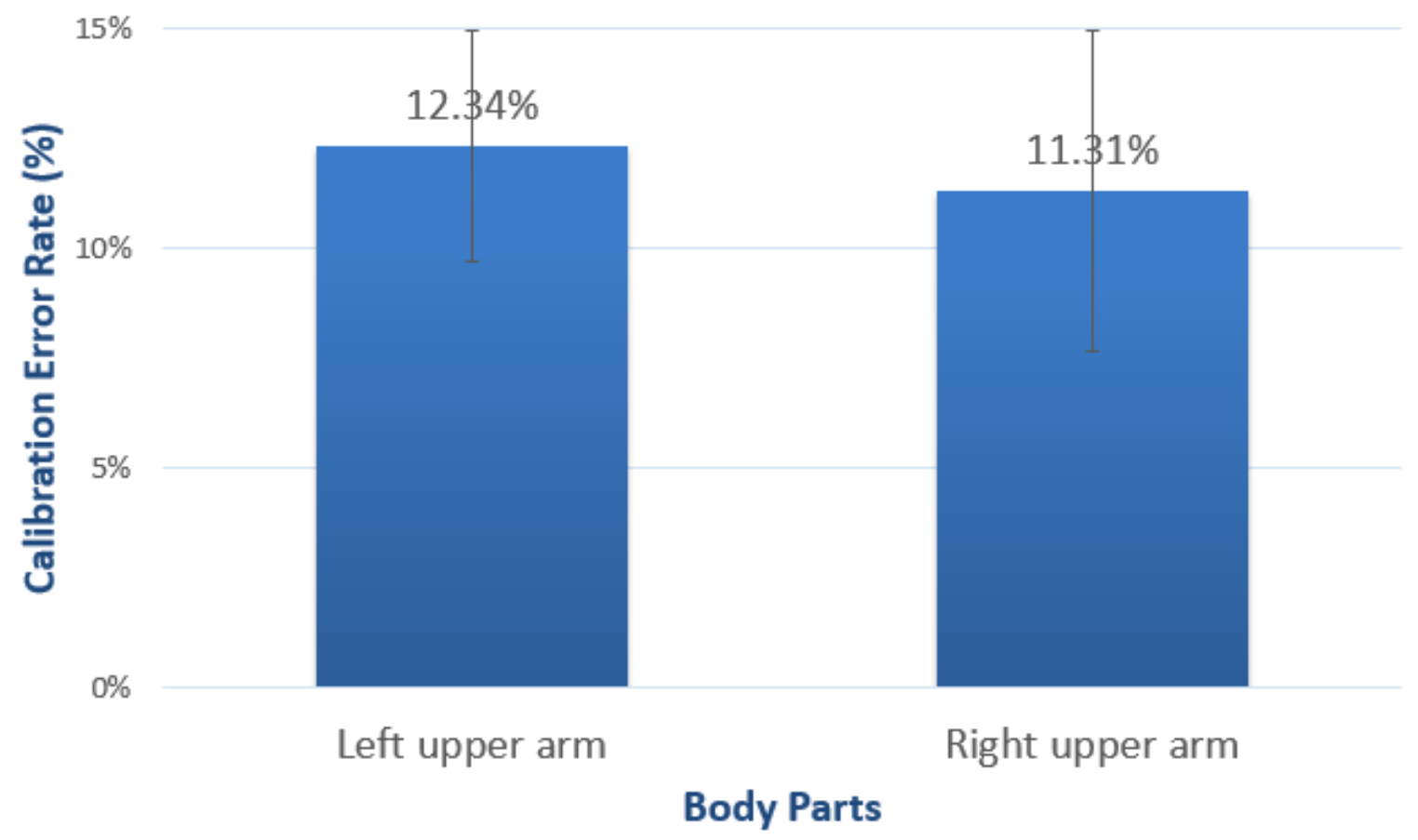

Figure 15. Upper arm length calibration average error rates (\%) for the left and right body sides across all participants.

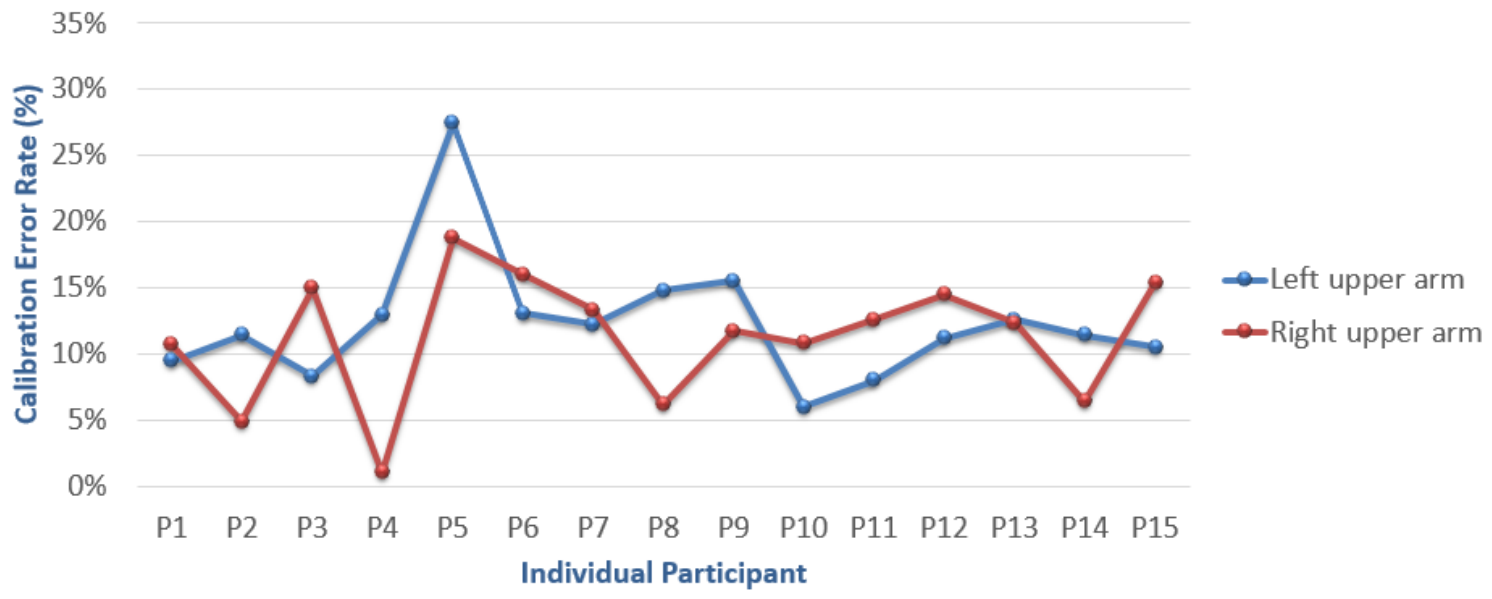

Figure 16. Upper arm length calibration error rates (\%) on the left and right body sides for individual participants. 
Table 3. Left upper arm length calibration detailed data for individual participants, including calculated length $(\mathrm{cm})$, real length $(\mathrm{cm})$, and error rates $(\%)$.

\begin{tabular}{|c|c|c|c|c|c|c|c|c|}
\hline \multicolumn{9}{|c|}{ Left Upper Arm } \\
\hline Participant & P1 & P2 & P3 & P4 & P5 & P6 & P7 & P8 \\
\hline Error Rate (\%) & $9.51 \%$ & $11.45 \%$ & $8.30 \%$ & $12.99 \%$ & $27.45 \%$ & $13.09 \%$ & $12.22 \%$ & $14.77 \%$ \\
\hline Calculated length $(\mathrm{cm})$ & 27.1 & 26.6 & 35.2 & 39.5 & 44.6 & 31.7 & 33.7 & 37.9 \\
\hline Real length $(\mathrm{cm})$ & 30.0 & 30.0 & 32.5 & 35.0 & 35.0 & 28.0 & 30.0 & 33.0 \\
\hline Participant & P9 & P10 & P11 & P12 & P13 & P14 & P15 & \\
\hline Error Rate (\%) & $15.52 \%$ & $6.00 \%$ & $8.01 \%$ & $11.22 \%$ & $12.61 \%$ & $11.44 \%$ & $10.50 \%$ & \\
\hline Calculated length $(\mathrm{cm})$ & 28.3 & 31.8 & 25.3 & 24.9 & 24.5 & 31.2 & 28.6 & \\
\hline Real length $(\mathrm{cm})$ & 33.5 & 30.0 & 27.5 & 28.0 & 28.0 & 28.0 & 32.0 & \\
\hline
\end{tabular}

Table 4. Right upper arm length calibration detailed data for individual participants, including calculated length $(\mathrm{cm})$, real length $(\mathrm{cm})$, and error rates $(\%)$.

\begin{tabular}{|c|c|c|c|c|c|c|c|c|}
\hline \multicolumn{9}{|c|}{ Right Upper Arm } \\
\hline Participant & P1 & P2 & P3 & P4 & P5 & P6 & P7 & P8 \\
\hline Error Rate (\%) & $10.67 \%$ & $4.87 \%$ & $15.00 \%$ & $1.02 \%$ & $18.79 \%$ & $16.00 \%$ & $13.30 \%$ & $6.21 \%$ \\
\hline Calculated length (cm) & 27.2 & 31.5 & 37.9 & 35.9 & 40.4 & 32.5 & 34.0 & 34.0 \\
\hline Real length $(\mathrm{cm})$ & 30.5 & 30.0 & 33.0 & 35.5 & 34.0 & 28.0 & 30.0 & 32.0 \\
\hline Participant & P9 & P10 & P11 & P12 & P13 & P14 & P15 & \\
\hline Error Rate (\%) & $11.74 \%$ & $10.82 \%$ & $12.61 \%$ & $14.46 \%$ & $12.34 \%$ & $6.39 \%$ & $15.43 \%$ & \\
\hline Calculated length $(\mathrm{cm})$ & 29.6 & 33.2 & 24.0 & 23.9 & 24.5 & 26.2 & 27.5 & \\
\hline Real length $(\mathrm{cm})$ & 33.5 & 30.0 & 27.5 & 28.0 & 28.0 & 28.0 & 32.5 & \\
\hline
\end{tabular}

\subsubsection{Torso Length Calibration}

In this proposed calibration protocol, torso length is calculated from both the left and right body sides based on the previously calibrated forearm and upper arm length, therefore, in the data analysis stage, calibrated torso length will be denoted respectively as the left torso and right torso. In doing so, it would be beneficial to compare and verify the torso length calibration accuracy between the left and right body sides. In terms of outputting the eventual torso length, the average value of the left and right calibrated torso length would be adopted.

As shown in Figure 17, the average calibration accuracy for the left and right torso are both satisfactory and vary within a reasonable range, showing the positive performance of the torso length calibration protocol in the situations that participants' body parts may not be exactly symmetrical and slight variations may exist between the left and right sensors. Figure 18, Table 5, and Table 6 further support and explain the trends discussed above. 


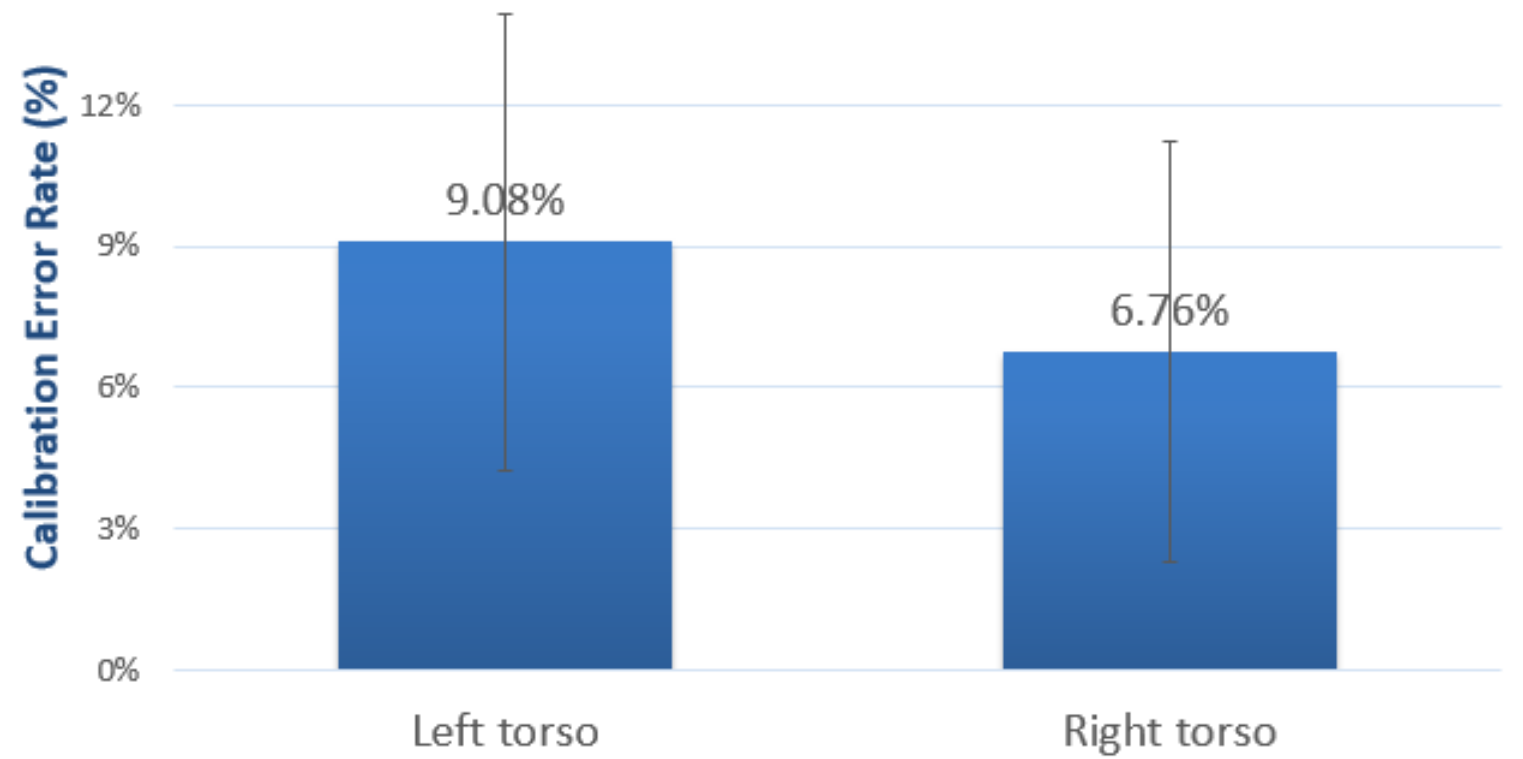

\section{Body Parts}

Figure 17. Torso length calibration average error rates (\%) for the left and right body sides across all participants.

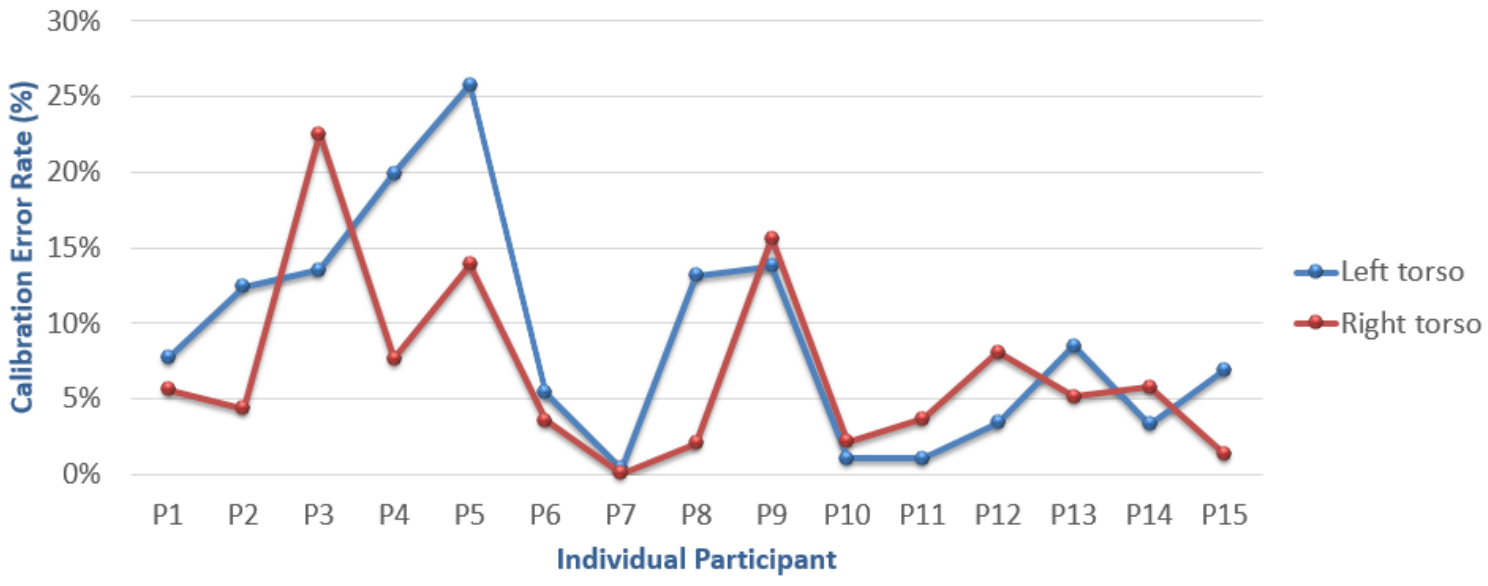

Figure 18. Torso length calibration error rates (\%) on the left and right body sides for individual participants. 
Table 5. Left torso length calibration detailed data for individual participants, including calculated length $(\mathrm{cm})$, real length $(\mathrm{cm})$, and error rates $(\%)$.

\begin{tabular}{|c|c|c|c|c|c|c|c|c|}
\hline \multicolumn{9}{|c|}{ Left Torso } \\
\hline Participant & P1 & P2 & P3 & P4 & P5 & P6 & P7 & P8 \\
\hline Error Rate (\%) & $7.73 \%$ & $12.42 \%$ & $13.51 \%$ & $19.89 \%$ & $25.76 \%$ & $5.39 \%$ & $0.37 \%$ & $13.17 \%$ \\
\hline Calculated length $(\mathrm{cm})$ & 46.1 & 45.1 & 48.8 & 58.7 & 63.5 & 38.8 & 49.7 & 49.8 \\
\hline Real length $(\mathrm{cm})$ & 50.0 & 51.5 & 43.0 & 49.0 & 50.5 & 41.0 & 49.5 & 44.0 \\
\hline Participant & P9 & P10 & P11 & P12 & P13 & P14 & P15 & \\
\hline Error Rate (\%) & $13.77 \%$ & $1.08 \%$ & $1.03 \%$ & $3.42 \%$ & $8.48 \%$ & $3.31 \%$ & $6.91 \%$ & \\
\hline Calculated length $(\mathrm{cm})$ & 50.6 & 49.5 & 43.4 & 41.0 & 38.4 & 43.4 & 49.2 & \\
\hline Real length (cm) & 44.5 & 49.0 & 43.0 & 42.5 & 42.0 & 42.0 & 46.0 & \\
\hline
\end{tabular}

Table 6. Right torso length calibration detailed data for individual participants, including calculated length $(\mathrm{cm})$, real length $(\mathrm{cm})$, and error rates $(\%)$.

\begin{tabular}{|l|c|c|c|c|c|c|c|c|}
\hline \multicolumn{9}{|c|}{ Right Torso } \\
\hline Participant & P1 & P2 & P3 & P4 & P5 & P6 & P7 & P8 \\
\hline Error Rate (\%) & $5.59 \%$ & $4.38 \%$ & $22.51 \%$ & $7.63 \%$ & $13.86 \%$ & $3.56 \%$ & $0.06 \%$ & $2.07 \%$ \\
\hline Calculated length (cm) & 47.2 & 49.2 & 52.1 & 53.3 & 57.5 & 43.0 & 49.0 & 44.9 \\
\hline Real length (cm) & 50.0 & 51.5 & 42.5 & 49.5 & 50.5 & 41.5 & 49.0 & 44.0 \\
\hline Participant & P9 & P10 & P11 & P12 & P13 & P14 & P15 & \\
\hline Error Rate (\%) & $15.63 \%$ & $2.17 \%$ & $3.68 \%$ & $8.09 \%$ & $5.12 \%$ & $5.75 \%$ & $1.31 \%$ \\
\hline Calculated length (cm) & 52.0 & 50.1 & 41.4 & 38.6 & 40.8 & 39.1 & 46.4 \\
\hline Real length (cm) & 45.0 & 49.0 & 43.0 & 42.0 & 43.0 & 41.5 & 47.0
\end{tabular}

\subsubsection{Whole Leg Length Calibration (Plus Foot/Shoe Height)}

As shown in Figure 19, the average calibration accuracy for the left and right whole leg (plus foot/shoe height) are both relatively accurate and also similar to each other, showing the robust performance of the whole leg length calibration protocol. As shown in Figure 20, when detailed comparing the whole leg length calibration accuracy between the left and right sides in terms of each participant, the error rate shows 3\%-10\% fluctuating trend, which could be due to the possible slight variations between both sides sensors, or the situation that calibration algorithm might be especially sensitive to sensor data input accuracy that slight difference in sensor data could lead to relatively large variations in error rate (for detailed discussion, please refer to section 6.5). Table 7 and Table 8 also support and illustrate the trends discovered above. 


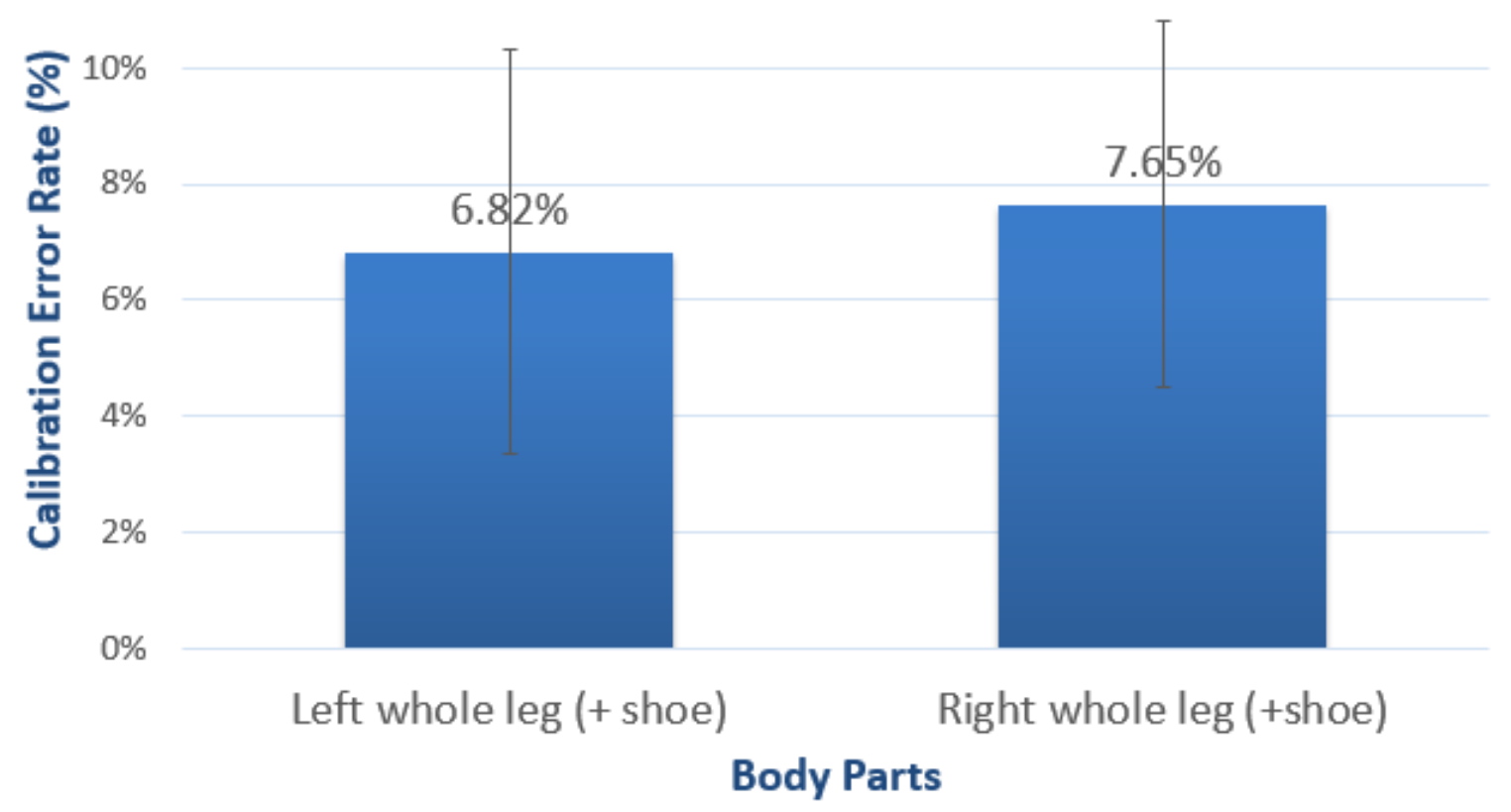

Figure 19. Whole leg length (plus foot/shoe height) calibration average error rates (\%) for the left and right body sides across all participants.

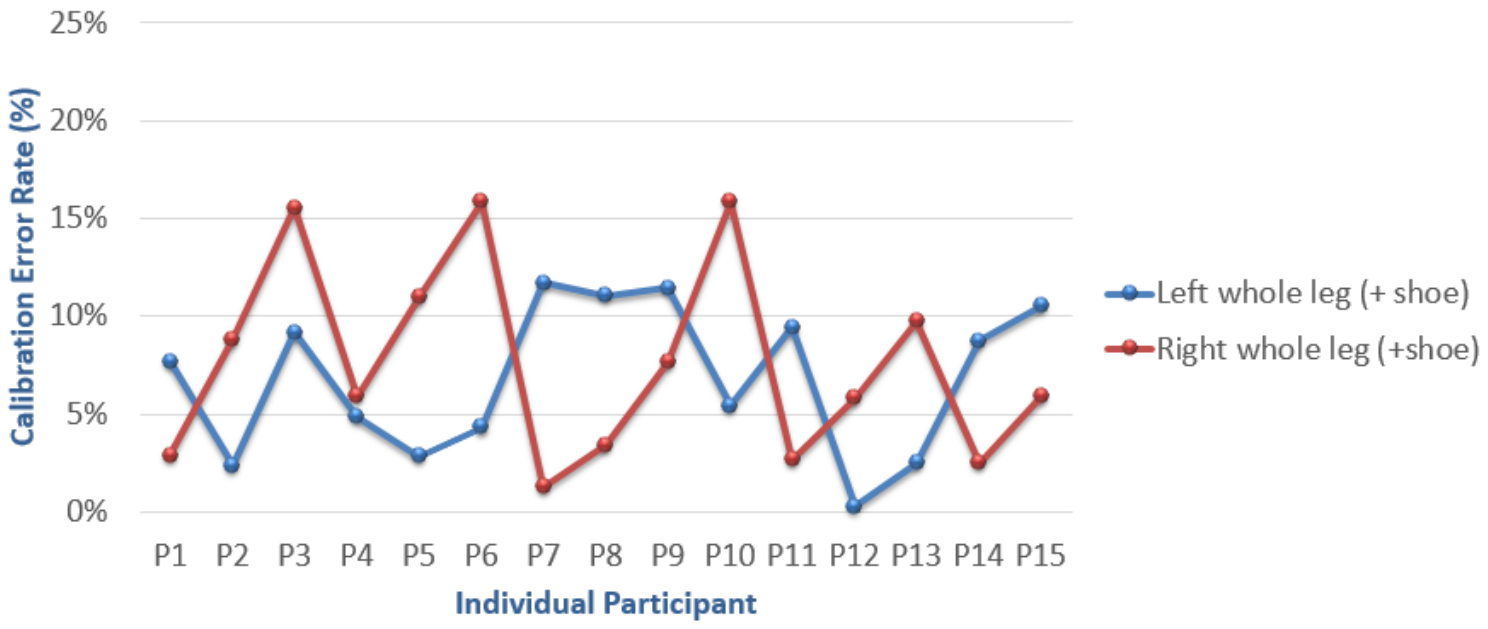

Figure 20. Whole leg length (plus foot/shoe height) calibration error rates (\%) on both left and right body sides for individual participants. 
Table 7. Left whole leg length (plus foot/shoe height) calibration detailed data for individual participants, including calculated length $(\mathrm{cm})$, real length $(\mathrm{cm})$, and error rates $(\%)$.

\begin{tabular}{|l|c|c|c|c|c|c|c|c|}
\hline \multicolumn{10}{|c|}{ Left Whole Leg (Plus Shoe Height) } \\
\hline Participant & P1 & P2 & P3 & P4 & P5 & P6 & P7 & P8 \\
\hline Error Rate (\%) & $7.68 \%$ & $2.36 \%$ & $9.15 \%$ & $4.87 \%$ & $2.86 \%$ & $4.32 \%$ & $11.72 \%$ & $11.07 \%$ \\
\hline Calculated length (cm) & 86.8 & 104.4 & 117.9 & 99.6 & 104.4 & 99.6 & 80.8 & 104.4 \\
\hline Real length (cm) & 94.0 & 102.0 & 108.0 & 95.0 & 101.5 & 95.5 & 91.5 & 94.0 \\
\hline Participant & P9 & $\mathbf{P 1 0}$ & $\mathbf{P 1 1}$ & $\mathbf{P 1 2}$ & $\mathbf{P 1 3}$ & $\mathbf{P 1 4}$ & $\mathbf{P 1 5}$ & \\
\hline Error Rate (\%) & $11.45 \%$ & $5.40 \%$ & $9.41 \%$ & $0.27 \%$ & $2.50 \%$ & $8.73 \%$ & $10.54 \%$ \\
\hline Calculated length (cm) & 86.8 & 90.8 & 90.8 & 80.8 & 86.8 & 80.8 & 86.8 \\
\hline Real length (cm) & 98.0 & 96.0 & 83.0 & 81.0 & 89.0 & 88.5 & 97.0 & \\
\hline
\end{tabular}

Table 8. Right whole leg length (plus foot/shoe height) calibration detailed data for individual participants, including calculated length $(\mathrm{cm})$, real length $(\mathrm{cm})$, and error rates $(\%)$.

\begin{tabular}{|l|c|c|c|c|c|c|c|c|}
\hline \multicolumn{9}{|c|}{ Right Whole Leg (Plus Shoe Height) } \\
\hline Participant & P1 & P2 & P3 & P4 & P5 & P6 & P7 & P8 \\
\hline Error Rate (\%) & $2.87 \%$ & $8.82 \%$ & $15.52 \%$ & $5.89 \%$ & $10.97 \%$ & $15.86 \%$ & $1.29 \%$ & $3.39 \%$ \\
\hline Calculated length (cm) & 90.8 & 112.1 & 90.8 & 90.8 & 90.8 & 80.8 & 90.8 & 90.8 \\
\hline Real length (cm) & 93.5 & 103.0 & 107.5 & 96.5 & 102.0 & 96.0 & 92.0 & 94.0 \\
\hline Participant & P9 & P10 & P11 & P12 & P13 & P14 & P15 & \\
\hline Error Rate (\%) & $7.63 \%$ & $15.86 \%$ & $2.68 \%$ & $5.83 \%$ & $9.75 \%$ & $2.50 \%$ & $5.89 \%$ \\
\hline Calculated length (cm) & 104.4 & 80.8 & 80.8 & 86.8 & 80.8 & 86.8 & 90.8 \\
\hline Real length (cm) & 97.0 & 96.0 & 83.0 & 82.0 & 89.5 & 89.0 & 96.5
\end{tabular}

\subsubsection{Shinbone Length Calibration (Plus Foot/Shoe Height)}

As shown in Figure 21, the average calibration accuracy for the left and right shinbone length (plus foot/shoe height) are both satisfactory and also similar to each other, showing the robust performance of the shinbone length calibration protocol. As shown in Figure 22, when comparing the shinbone length (plus foot/shoe height) calibration accuracy between the left and right sides in terms of each participant, the error rate shows fluctuations, similar to the discussion in section 4.2.4, which could also possibly be due to the situation that calibration algorithm might be sensitive to sensor data input accuracy (for detailed discussion, please refer to section 6.5). Table 9 and Table 10 also detailed support the above trends.

Please note, P8 (participant No.8) is a noisy data point in terms of the shinbone length calibration because P8's ankle was injured and worn auxiliary rehabilitation protective covers on his ankles, so when attaching the sensors on his ankles, the sensor's direction cannot be very accurate to reflect the direction of his shinbone, resulting in the 
calibration of his shinbone length appears a great error. Therefore P8's data has been excluded in this section, please check Appendix C.1 for P8's detailed data.

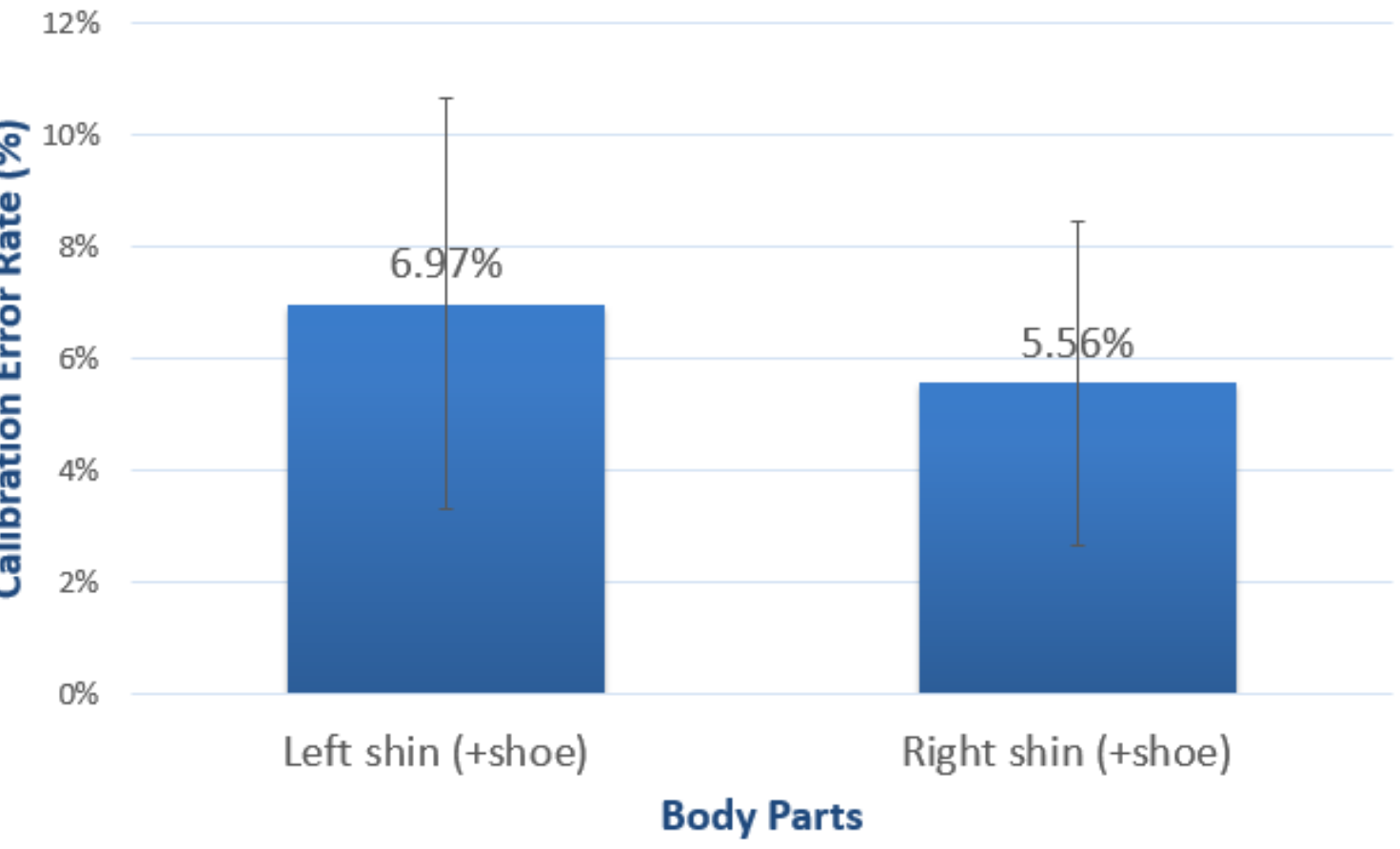

Figure 21. Shinbone length (plus foot/shoe height) calibration average error rates (\%) for the left and right body sides across all participants.

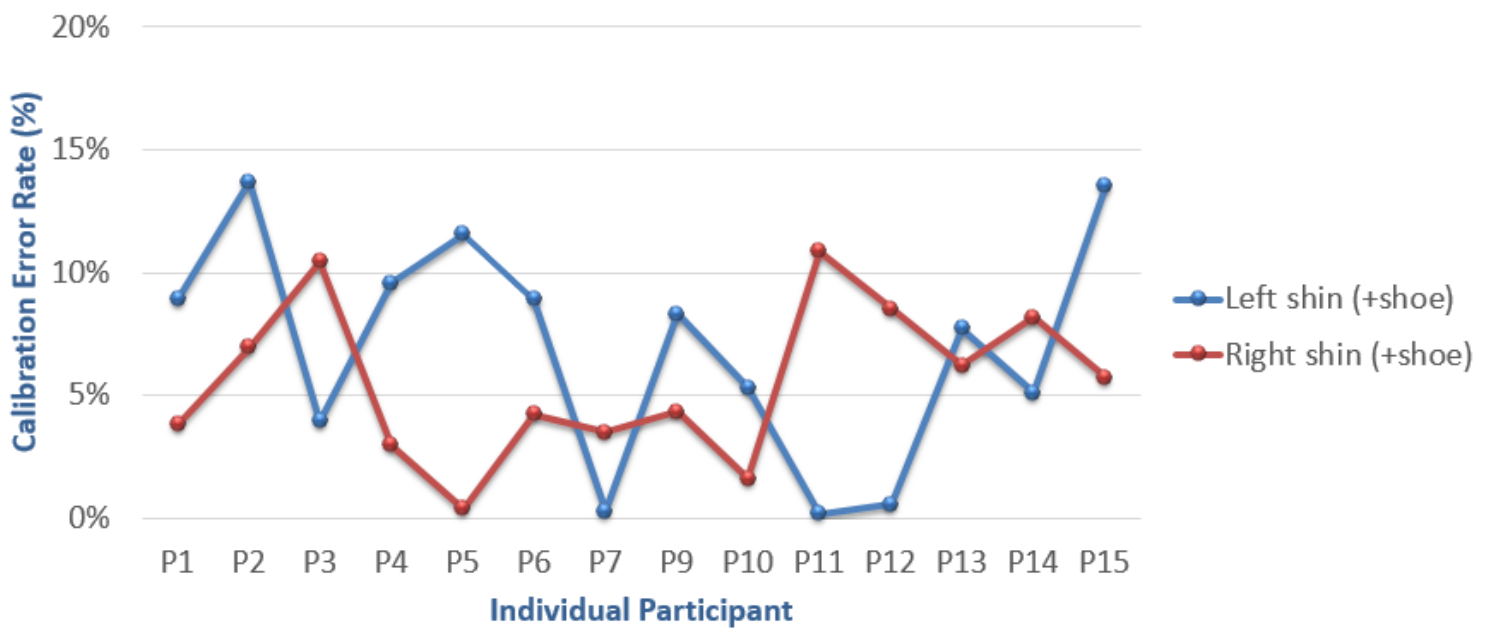

Figure 22. Shinbone length (plus foot/shoe height) calibration error rates (\%) on the left and right body sides for individual participants. 
Table 9. Left shinbone length (plus foot/shoe height) calibration detailed data for individual participants, including calculated length $(\mathrm{cm})$, real length $(\mathrm{cm})$, and error rates $(\%)$.

\begin{tabular}{|l|c|c|c|c|c|c|c|}
\hline \multicolumn{7}{|c|}{ Left Shin (Plus Shoe Height) } \\
\hline Participant & P1 & P2 & P3 & P4 & P5 & P6 & P7 \\
\hline Error Rate (\%) & $8.92 \%$ & $13.67 \%$ & $3.94 \%$ & $9.57 \%$ & $11.57 \%$ & $8.91 \%$ & $0.29 \%$ \\
\hline Calculated length (cm) & 51.5 & 43.2 & 56.6 & 57.0 & 45.1 & 45.5 & 46.1 \\
\hline Real length (cm) & 56.5 & 50.0 & 54.5 & 52.0 & 51.0 & 50.0 & 46.0 \\
\hline Participant & P9 & P10 & P11 & P12 & P13 & P14 & P15 \\
\hline Error Rate (\%) & $8.31 \%$ & $5.30 \%$ & $0.19 \%$ & $0.58 \%$ & $7.70 \%$ & $5.12 \%$ & $13.51 \%$ \\
\hline Calculated length (cm) & 49.1 & 49.2 & 42.9 & 45.3 & 42.9 & 45.1 & 45.8 \\
\hline Real length (cm) & 53.5 & 52.0 & 43.0 & 45.0 & 46.5 & 47.5 & 53.0 \\
\hline
\end{tabular}

Table 10. Right shinbone length (plus foot/shoe height) calibration detailed data for individual participants, including calculated length $(\mathrm{cm})$, real length $(\mathrm{cm})$, and error rates $(\%)$.

\begin{tabular}{|l|c|c|c|c|c|c|c|}
\hline \multicolumn{7}{|c|}{ Right Shin (Plus Shoe Height) } \\
\hline Participant & P1 & P2 & P3 & P4 & P5 & P6 & P7 \\
\hline Error Rate (\%) & $3.79 \%$ & $6.97 \%$ & $10.46 \%$ & $2.96 \%$ & $0.40 \%$ & $4.24 \%$ & $3.50 \%$ \\
\hline Calculated length (cm) & 59.2 & 55.6 & 59.1 & 50.5 & 50.7 & 47.4 & 48.1 \\
\hline Real length (cm) & 57.0 & 52.0 & 53.5 & 52.0 & 50.5 & 49.5 & 46.5 \\
\hline Participant & P9 & P10 & P11 & P12 & P13 & P14 & P15 \\
\hline Error Rate (\%) & $4.35 \%$ & $1.61 \%$ & $10.85 \%$ & $8.54 \%$ & $6.23 \%$ & $8.17 \%$ & $5.74 \%$ \\
\hline Calculated length (cm) & 51.2 & 53.9 & 48.2 & 40.2 & 49.4 & 42.7 & 50.0 \\
\hline Real length (cm) & 53.5 & 53.0 & 43.5 & 44.0 & 46.5 & 46.5 & 53.0 \\
\hline
\end{tabular}

\subsection{Calibration Time Duration}

In this experiment, the average calibration time duration across all participants is 22.4 minutes, and the calibration time duration for each participant varies within the range from 18 to 29 minutes, as shown in Figure 23. In terms of calibration for different body parts, as shown in Figure 24, the forearm calibration is the fastest to accomplish, which on average accounts for $13.33 \%$ of the entire calibration time duration, while the calibration for upper arm and torso would consume a relatively larger time proportion, accounting for $37.78 \%$ of the entire calibration duration because it consists of comparably more complicated calibration requirements (for detailed calibration time consumption discussion, please refer to section 6.6). 


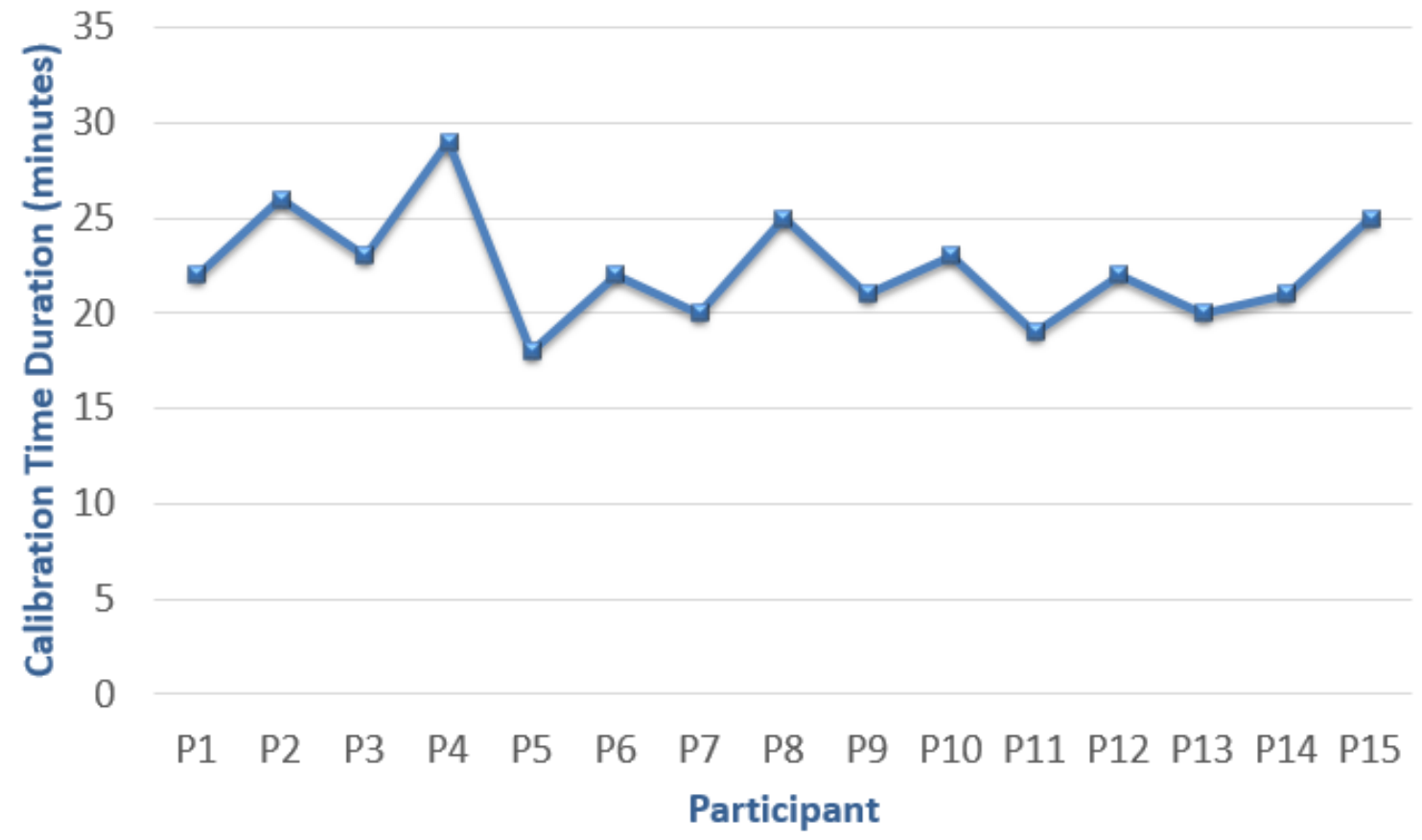

Figure 23. Total calibration time duration (minutes) for individual participants.

\section{Calibration Time Proportion for Body Parts (\%)}

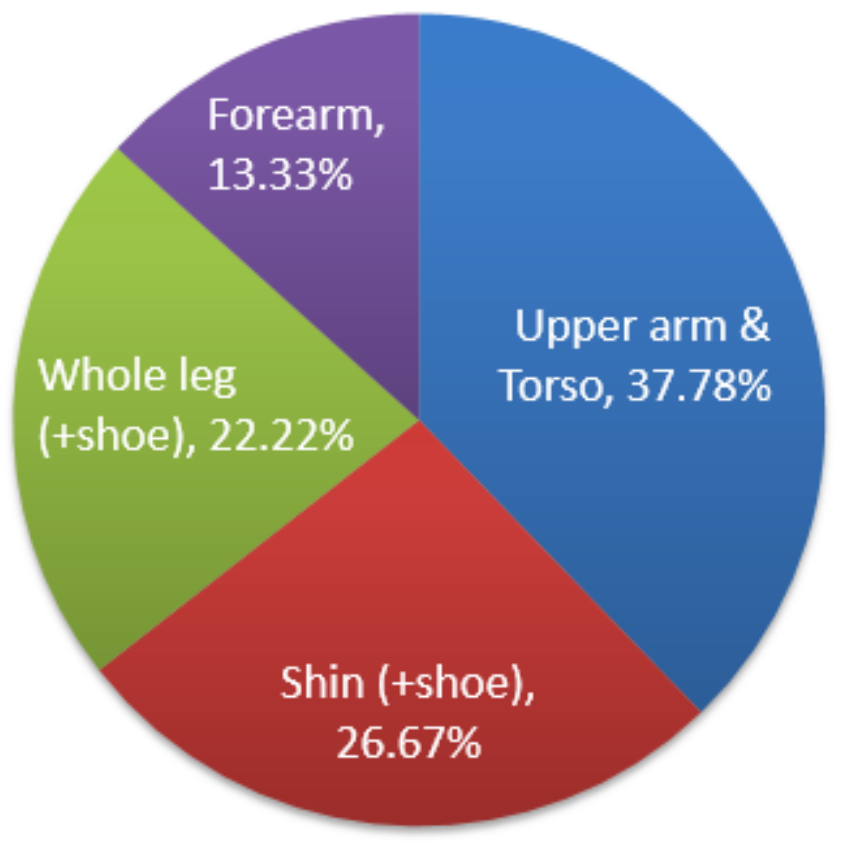

Figure 24. Average calibration time proportion (\%) of different body parts across all participants. 


\section{Chapter: Sample Interface}

\subsection{Brief Introduction}

In this chapter, a calibration auxiliary system is proposed, which contains several sample interfaces involving all the required calibration poses and requirements designed in this thesis. This calibration system contains a Pose Monitor system, which can dynamically track users' poses to evaluate if their pose meets requirements, and the Pose Monitor system can also reflect users' current pose position in real time through real-time visualization in this interface to make users aware of how to adjust a pose to meet requirements.

The reason for designing this calibration auxiliary system is that, in the user research process, in order to convey and explain the calibration poses requirements, each participant was provided with the printed three-perspective pose illustration images and the researcher's oral instructions. It was found that this would cost excessive time, and may not be convenient for participants to fully understand the key points of the poses, and sometimes participants' poses could be difficult to meet the requirements because they could not see their own pose so they would not be aware of how they are performing. In these cases, the researcher would have to manually adjust participants' poses in order to meet the pose requirements, which could potentially create uneasy or unpleasant feelings for some participants.

Therefore, the objectives of designing this calibration auxiliary system are to make the entire calibration process clearer, more efficient, friendlier, and to provide more information to users. Through the proposed Pose Monitor system, users could intuitively understand the requirements of each pose and conveniently evaluate their own pose through the dynamic and real-time reflection in the interface, so they could adjust their pose by themselves (without assistance from the researcher), which is expected to enhance the efficiency and effectiveness of the calibration process, and to make users experience more fun and feel friendlier, more informed, more interactive, and more active (for detailed mechanism of the Pose Monitor system, please refer to section 5.2.2). 


\subsection{Sample Interface}

\subsubsection{Welcome and General Introduction Sample Interface}

This is the welcome and general introductions sample interface for this proposed auxiliary calibration system, as shown in Figure 25, which consists of a general introduction page, and the Pose 1 preparation and transition page.

In the general introduction page, as shown in Figure 25 (a), users will be informed of the number of poses that they will be required to perform, the estimated time it will probably take, and the Pose Monitor system that will be used in the calibration process to dynamically track, reflect, and evaluate their poses and automatically indicate if their pose meets the requirements or still needs to be adjusted (for detailed mechanism of the Pose Monitor system, please refer to section 5.2.2). This general introduction page is aimed at providing users with a holistic overview of the entire calibration process to make users feel better informed, confident, and relaxed.

In the Pose 1 preparation and transition page, as shown in Figure 25 (b), the system will present the general image of Pose 1 to make users aware that the calibration process is about to formally start, which would also be helpful for users to develop an impression about what Pose 1 would look like. If ready, users can proceed and initiate the process by clicking the "NEXT" button on this page.

(a)

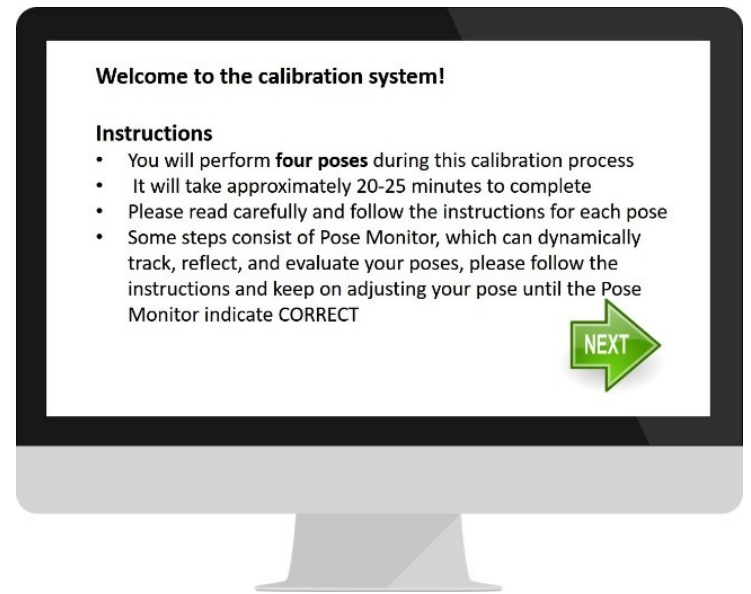

(b)

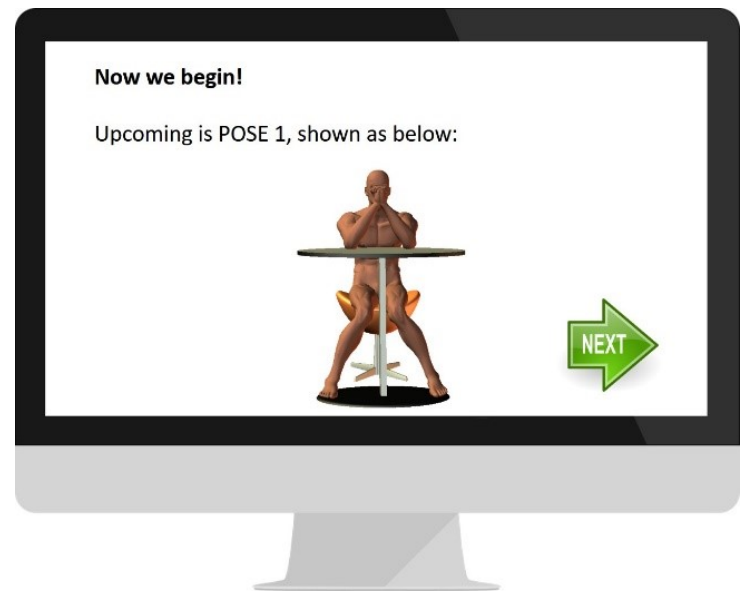

Figure 25. Calibration auxiliary system introduction sample interface: (a) Welcome and general introduction page; (b) Preparation and transition to Pose 1. 


\subsubsection{Pose 1 Forearm Length Calibration Sample Interface}

As shown in Figure 26, the sample interfaces for Pose 1 the forearm length calibration is comprised of four parts, including the interface (a) step 1, which informs users that they should wear sensors on their designated body parts, and the general requirements for this calibration step; Interface (b) and (c), the possible situations in step 2, which consist of the Pose Monitor system to interactively and automatically reflect, evaluate, and indicate if users properly performed the required pose. After the Pose Monitor system indicates that user's pose meets the requirements, users will be instructed by this interface that he should hold this pose still and it will take some time to measure and record sensor data; Interface (d), a transition page, which will be presented to users when the previous step is done to make users aware that the Pose 1 calibration has been completed, and help users to get a sense of what the next calibration pose will be.

The mechanism of the proposed Pose Monitor system, as shown in Figure 26 (b) and (c), is that the system will dynamically monitor the tri-axial accelerometers data from the sensors that users are wearing. Pose 1 requires users' forearms to be perpendicular to the horizontal table, which requires $\mathrm{Y}$-axis values of the left and right accelerometer sensors read $\mathrm{Y}=0 \mathrm{G}$ (for the reason why requiring $\mathrm{Y}=0 \mathrm{G}$, please refer to section 3.3.1 and section 3.4.1). Through dynamically tracking users' accelerometers data (from sensors on their wrists), the Pose Monitor can track the forearms' real-time orientation and position, automatically identify if users' forearms are perpendicular to the horizontal table, and in real time will reflect users' current forearm position through synchronously altering the forearm position of the character in the interface. This mechanism could help to conveniently and intuitively indicate to users if their pose is acceptable or not, and how they should adjust their pose. If the pose is correctly performed, the proposed interface will automatically present a CHECK mark, a CORRECT sign, forearms in normal skin color, further instructions, as well as a colored "NEXT" button. In contrast, if user's pose is incorrect, the Pose Monitor will grey out and disable the "NEXT" button, and automatically present users with a CROSS mark, a WRONG sign, and pale-colored forearms (which can be easily distinguished from normal skin color) to remind users that their pose is still needed to be adjusted to meet the requirements. 
(a)

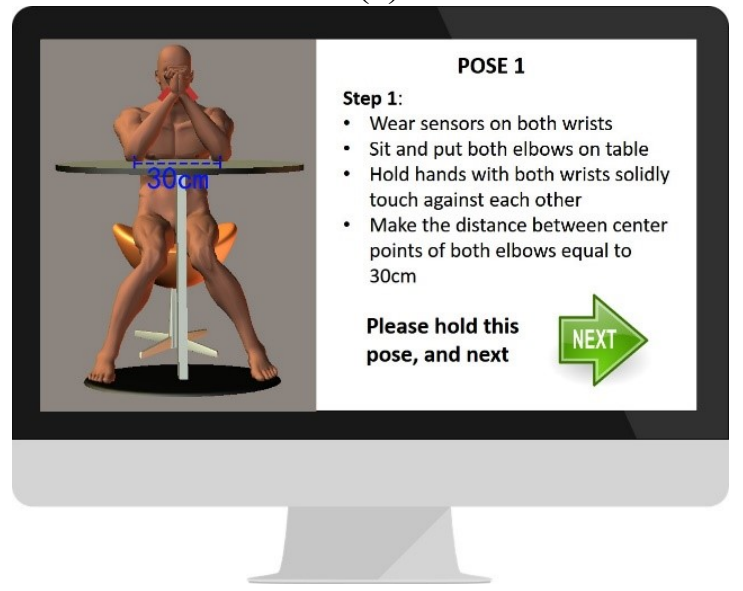

(c)

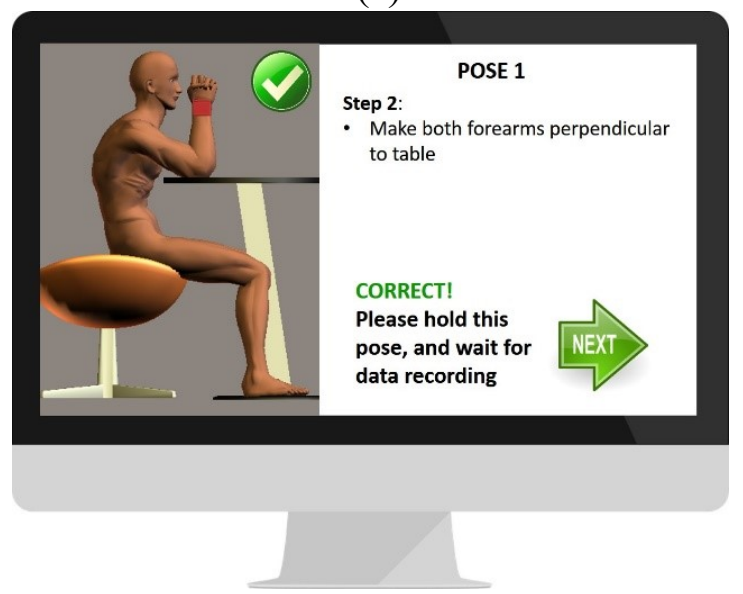

(b)

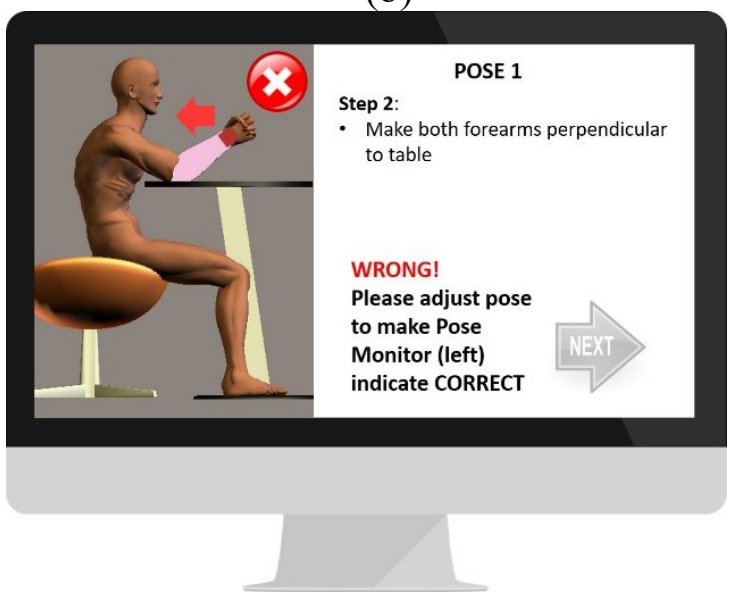

(d)

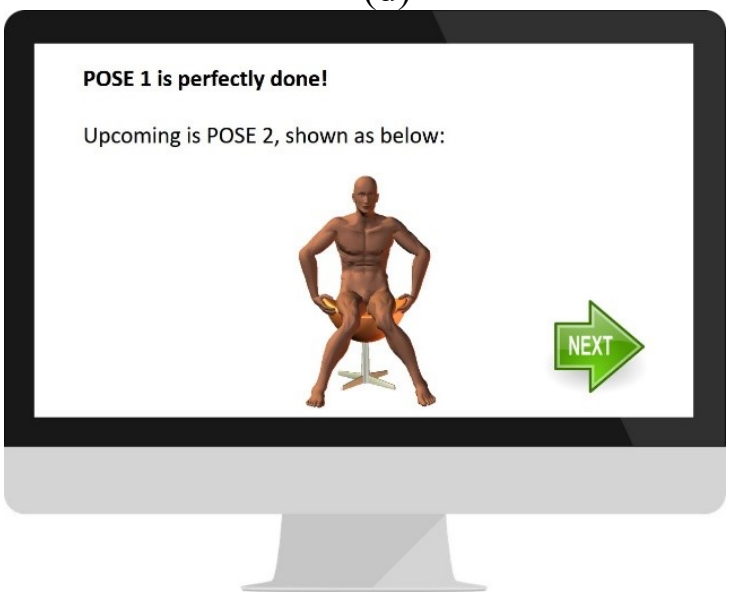

Figure 26. Pose 1 forearm length calibration auxiliary system sample interface: (a) Step 1; (b), (c) Step2: Pose Monitor to dynamically track, reflect, and evaluate user's pose (the situations of the correct and wrong poses); (d) Preparation and transition to Pose 2.

\subsubsection{Pose 2 Shinbone Length Calibration Sample Interface}

The calibration interface for Pose 2 basically shares the consistent design ideas with the interface of Pose 1. As shown in Figure 27, Pose 2 the shinbone length calibration interfaces contains five parts, including the interface (a) step 1, which is for initial pose instructions, interface (b) and (c), comprised of Pose Monitor to dynamically reflect and evaluate users' pose performance, interface (d), which presents further instructions for Pose 2 and allows time for data recording, and interface (e), a transition page to the next pose.

Similar to the working principle and design ideas for Pose 1, the Pose Monitor system for Pose 2, as shown in Figure 27 (b) and (c), can help to automatically identify if a user's pose meets the requirement that both shinbones should be perpendicular to the horizontal ground. This is realized through tracking Y-axis accelerometer readings from 
the sensors worn on the lateral sides of user's ankles, and the interface will indicate that user's pose is correct when the $\mathrm{Y}$-axis values of the left and right accelerometers read $\mathrm{Y}=0$ G. Otherwise, users still need to adjust their shinbones' position to meet the requirements.

(a)

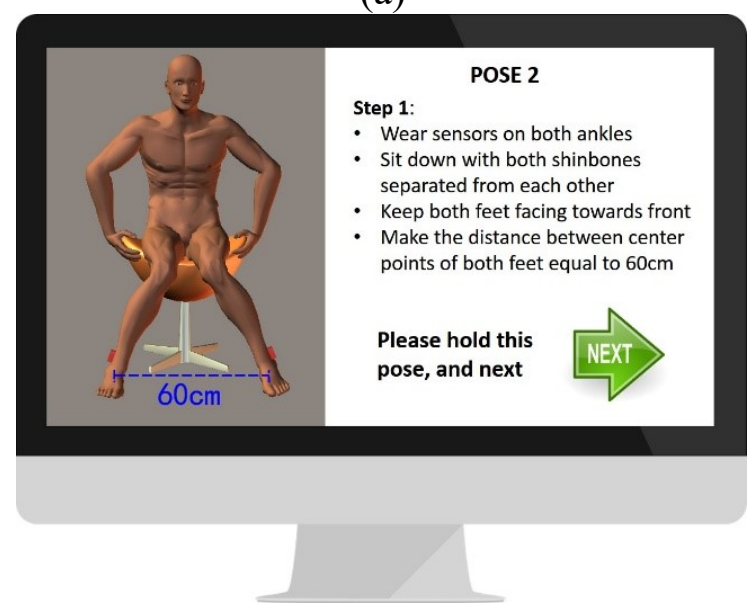

(c)

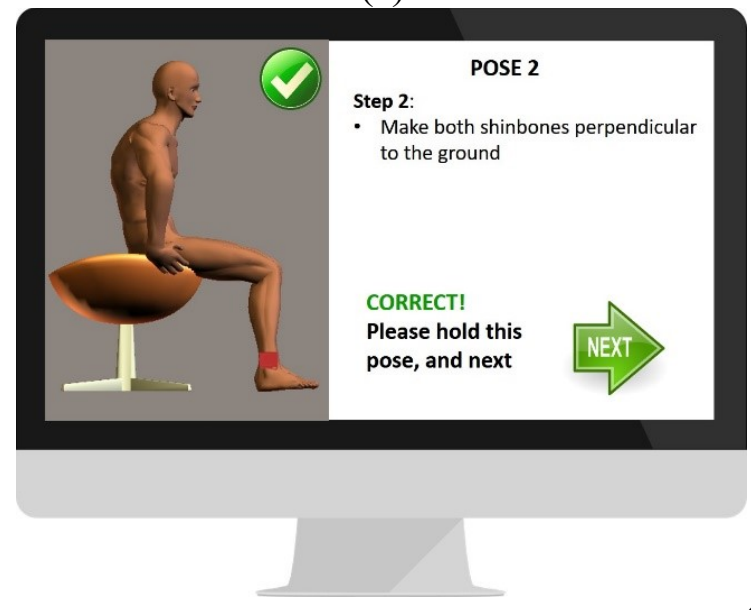

(b)

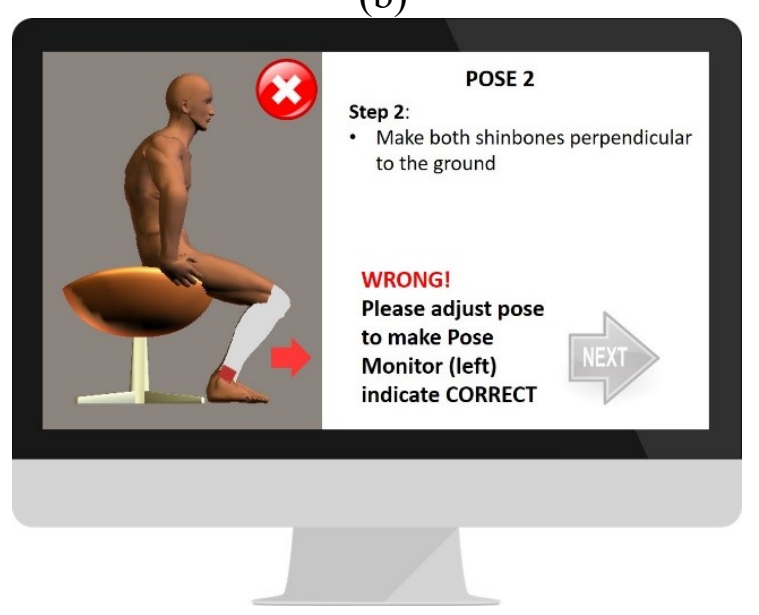

(d)

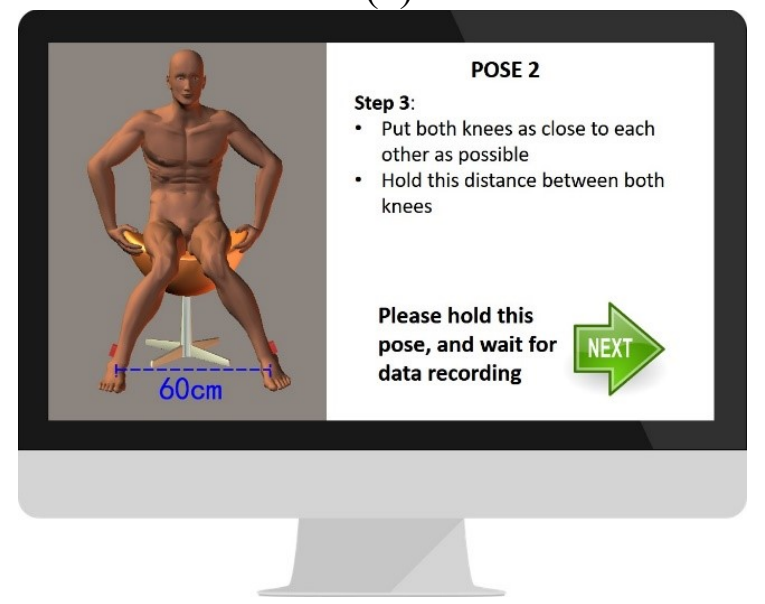

(e)

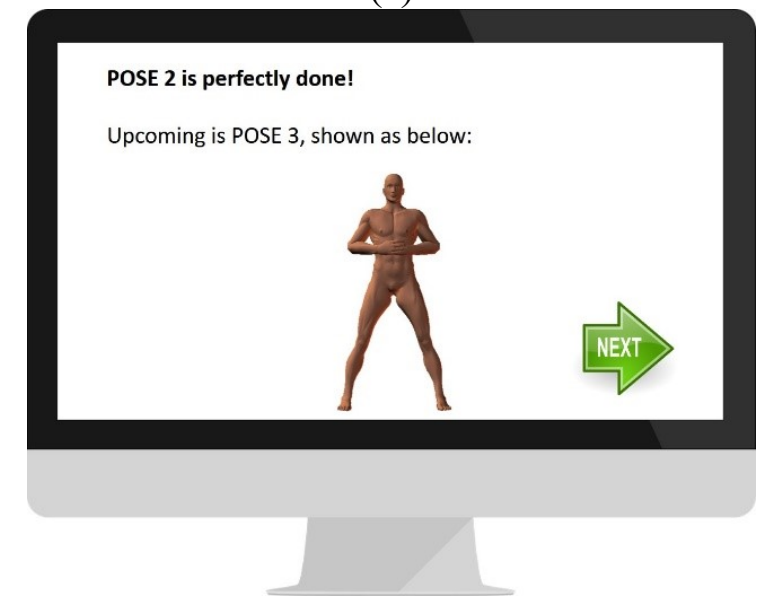

Figure 27. Pose 2 shinbone length calibration system sample interface: (a) Step 1; (b), (c) Step2: Pose Monitor system to dynamically track, reflect, and evaluate user's pose (the situations of the correct and wrong poses); (d) Step 3; (e) Preparation and transition to Pose 3. 


\subsubsection{Pose 3 Whole Leg Length Calibration Sample Interface}

As shown in Figure 28, Pose 3 the whole leg length calibration interface also contains four parts, including the interface (a) step 1, which is for general instructions, the interface (b) and (c) containing the Pose Monitor system to interactively reflect and evaluate if the required pose is properly performed, and the interface (d), which is a transition page to the next pose.

Sharing the common working principle and design ideas with Pose 1, the Pose Monitor for Pose 3, as shown in Figure 28 (b) and (c), can help to automatically identify if both of the user's legs are perpendicular to the horizontal ground, which is one of the requirements of Pose 3. To realize it, the Pose Monitor system will track accelerometer Yaxis readings from the sensors worn on the lateral side of users' left and right knees, and when the $\mathrm{Y}$-axis values of both accelerometer sensors read $\mathrm{Y}=0 \mathrm{G}$, the interface will indicate that the user correctly performs the pose. In contrast, if user's pose is not correct, the interface will show pale color legs, a CROSS mark, and a WRONG sign to make the user aware that the pose needs adjustment. 
(a)

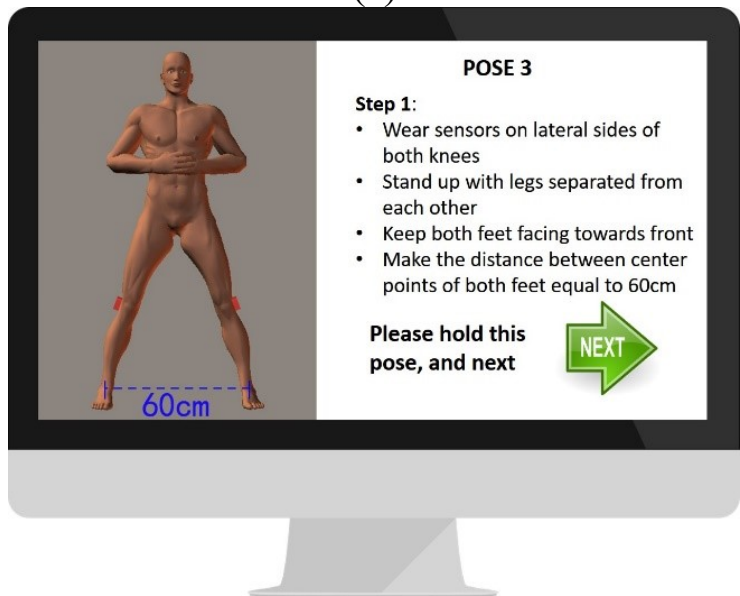

(c)

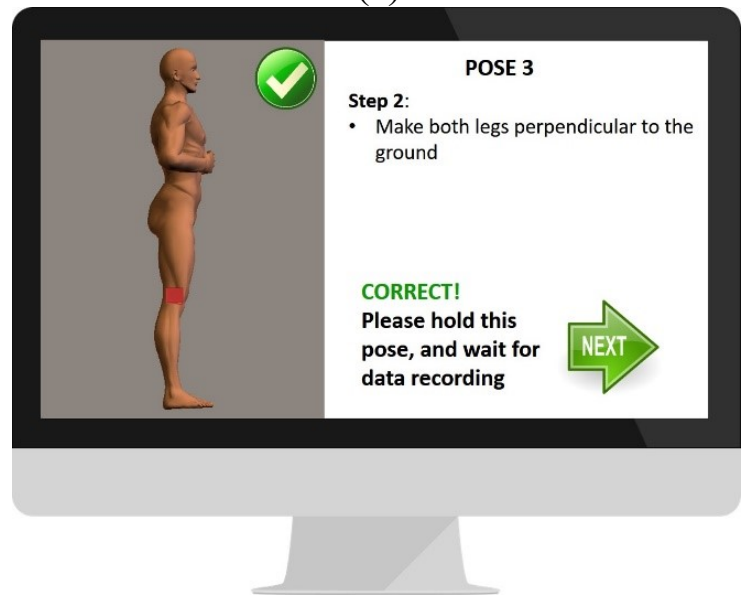

(b)

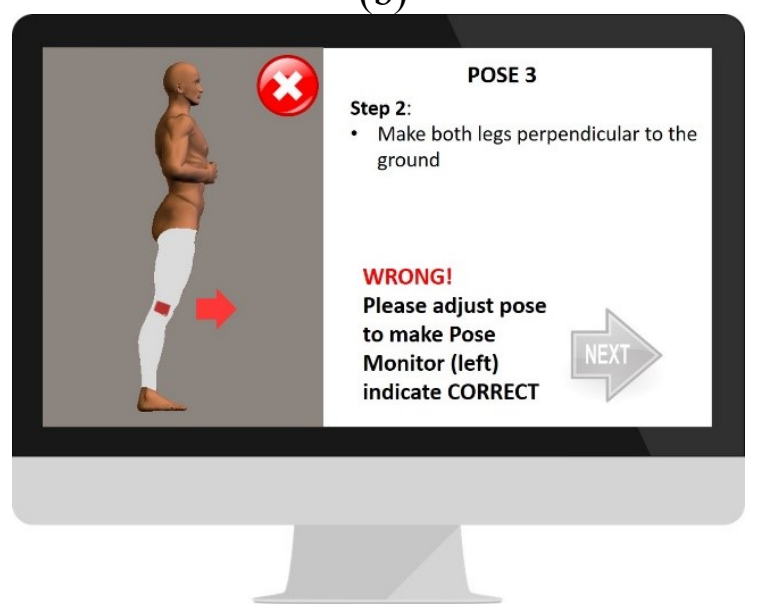

(d)

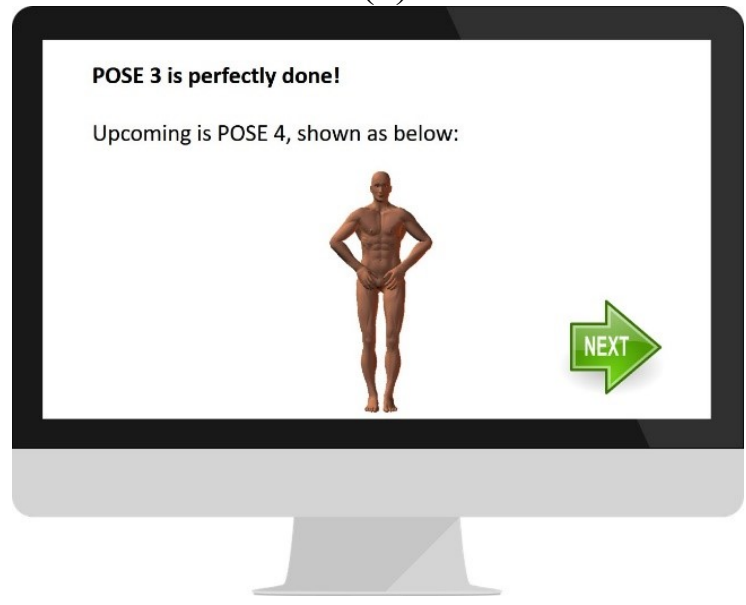

Figure 28. Pose 3 whole leg length calibration system sample interface: (a) Step 1; (b), (c) Step2: Pose Monitor system to dynamically track, reflect, and evaluate user's pose (the situations of the correct and wrong poses); (d) Preparation and transition to Pose 4.

\subsubsection{Pose 4 Upper Arm and Torso Length Calibration Sample Interface}

Similar to the previous poses, Pose 4 the upper arm and torso length calibration interface, as shown in Figure 29, contains four parts, including the interface (a) step 1, which is for general instructions, the interface (b) and (c), which contain the Pose Monitor system, and the interface (d), which is the ending page of the entire calibration process.

The Pose Monitor for Pose 4, as shown in Figure 29 (b) and (c), can help to automatically identify and indicate users whether their pose meet the requirement of Pose 
4 that on each side, the upper arm and forearm should be kept in a straight line, and the flat of upper arm and forearm should be perpendicular to the horizontal ground.

The mechanism is as follows. The Pose Monitor system will track accelerometer Z-axis readings from the sensors worn on users' wrists and upper arms. On each side, when Z-axis values of accelerometer sensors on both wrist and upper arm simultaneously read $\mathrm{Z}=0 \mathrm{G}$, the interface will indicate that user's pose is correct. Otherwise, user's pose is incorrect, and the interface will indicate to the user that the pose needs to be adjusted.

(a)

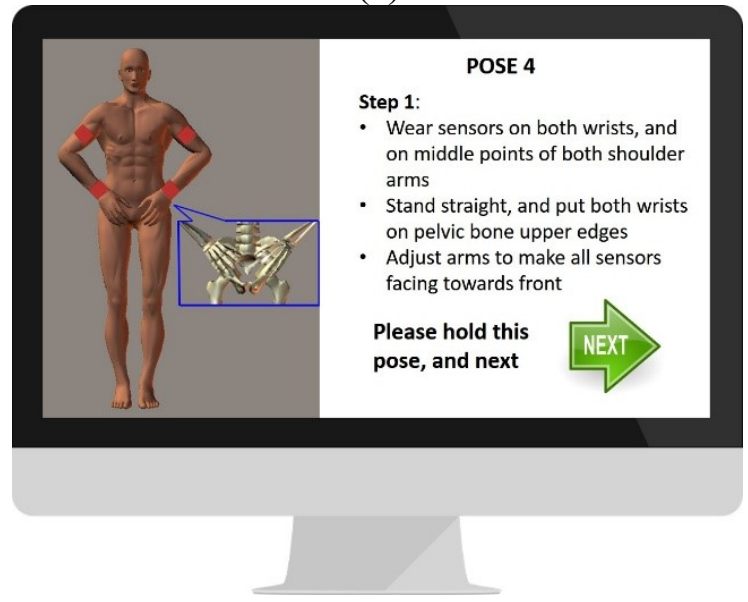

(c)

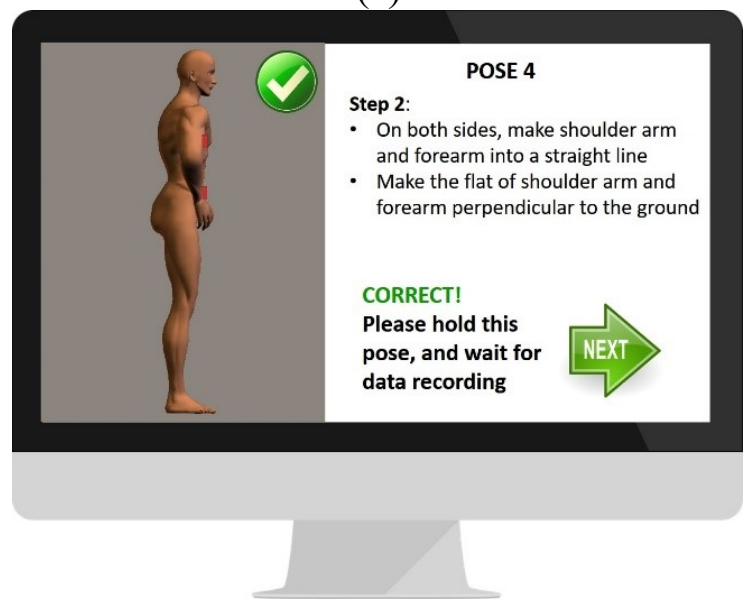

(b)

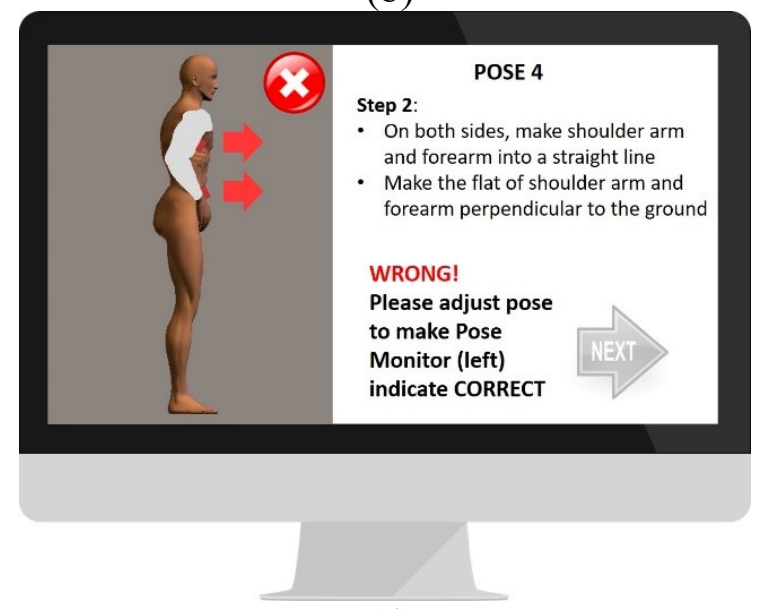

(d)

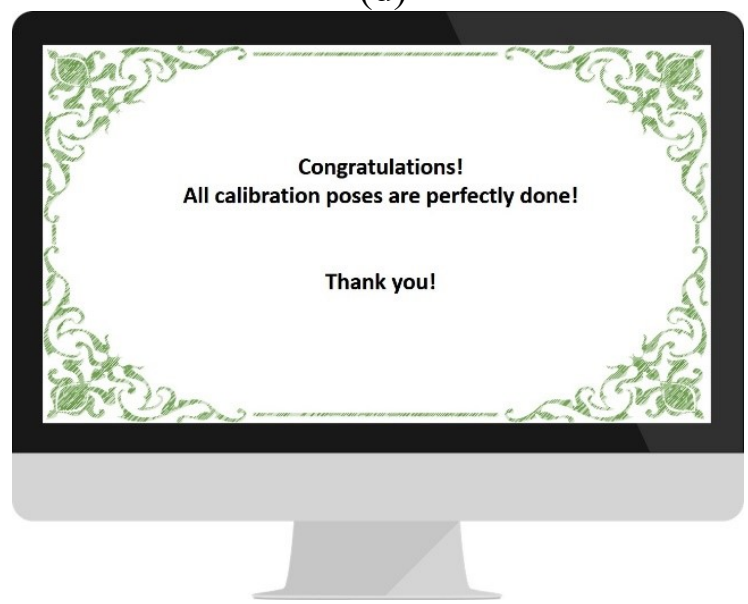

Figure 29. Pose 4 upper arm and torso length calibration system sample interface: (a) Step 1; (b), (c) Step2: Pose Monitor system to dynamically track, reflect, and evaluate user's pose (the situations of the correct and wrong poses); (d) Ending page. 


\section{Chapter: Discussion}

\subsection{Errors}

In this experiment, the proposed calibration system provides satisfactory calibration accuracy, and the calibration errors have been controlled within a reasonable range, with an overall $7.64 \%$ average calibration error rate across all body parts and all participants. This calibration error rate is acceptable because calibration errors came from multiple factors in this experiment, including the following aspects: sensor placement may not perfectly align with the direction of body parts, the participants' pose may not fully fulfill the requirements, the sensor noise generated by the circuit board or the surroundings, the limit of sensor reading numerical precision, the cumulative errors due to the pose and algorithm design, and the manually measurement of body parts' length could also generate errors. When integrating all the factors that could potentially generate errors, the overall 7.64\% average calibration error rate in this experiment is not so large and considered acceptable. In addition, considering the length of human body parts, when calculating using this inertial sensor-based calibration methodology, 7.64\% average calibration error rate would only generate a few centimeters calibration difference between the calculated and the actual body parts' length, which would not largely influence its utility and effectiveness.

When considering the calibration error rates for different body parts, upper arm calibrations generate relatively more errors when compared to the other body parts' calibration results, which on the one hand may because the calibration pose for the upper arm is comparably more complicated for participants, and on the other hand, may also be due to the cumulative errors, which is to say that the upper arm length calibration algorithm is based on the already calibrated forearm length in the previous step, so upper arm calibration errors would also involve the forearm calibration errors. The other error factors mentioned above will be discussed in detail in the following sections.

\subsection{Sensor Placement}

The positions where sensors are fixed on the user's body are critical to the calibration accuracy. Aimed at calculating a user's main skeletal structure length 
parameters, the sensors' placement should align with user's skeletal orientations as perfectly as possible, thereby calculating the designated body part length based on the angle calculated from the sensor readings. Therefore, the better the alignment between the sensors and the bones, the more accurate the calculated angle and the calibrated body part length would be. However, besides bones, the human body also consists of massive muscles and fat, which makes it more challenging to place sensors to exactly reflect the bones' directions, instead of mistakenly reflecting the orientations and angles of the muscles.

In this proposed calibration protocol, the positions of sensor placement are all meticulously designed to place sensors on the spots where bodies have the least muscles, so it would be relatively easiest to reflect the directions of the designated body part bones. For example, when calibrating the forearm length, the sensors are placed on the wrists instead of the elbow or the mid-forearm because wrist contains the least muscle and fat in the entire forearm area, which is also the same reason why sensors are placed on the lateral side of knees when calibrating whole leg length.

\subsection{Calibration Pose Symmetrical Requirements}

In order to accurately calculate the body part length, users need to closely fulfill the requirements of each calibration pose, and they should try to ensure the pose actions are symmetrical on both left and right body sides, otherwise, the non-symmetrical poses on both sides may decrease calibration accuracy. For instance, as shown in Figure 30, when calibrating the forearm length, due to the symmetry nature of the left and right sides, the distance between both elbows $(\mathrm{ac}=30 \mathrm{~cm})$ is simply equally divided in half $(\mathrm{ab}=\mathrm{bc}=$ $15 \mathrm{~cm}$ ) to be further respectively used as a parameter to calculate the left and right forearm length. However, this could only be realized when the forearms' poses and directions are symmetrical on the left and right sides, otherwise, if not symmetrical, it could not simply divide the distance between both elbows (ac) in half, and it would take much more effort to measure the distance of $a b$ and $b c$ (as shown in Figure 30) because the point $b$ would no longer be the center point between point a and point $c$, and the point $b$ might skew towards either point a or point c. Therefore, in order to increase the calibration accuracy and simplify the calibration procedure, user's pose performing should be symmetrical on the 
left and right body sides. In addition, although human body parts' length on both sides also might not be exactly identical, for the physically normal user group, there would only exist very slight variations between the left and right body structure, which would not seriously affect the calibration accuracy. Therefore, in order to simplify the calibration procedure, for the physically normal user group, we can deem the human body structure and length as symmetrical on the left and right sides.

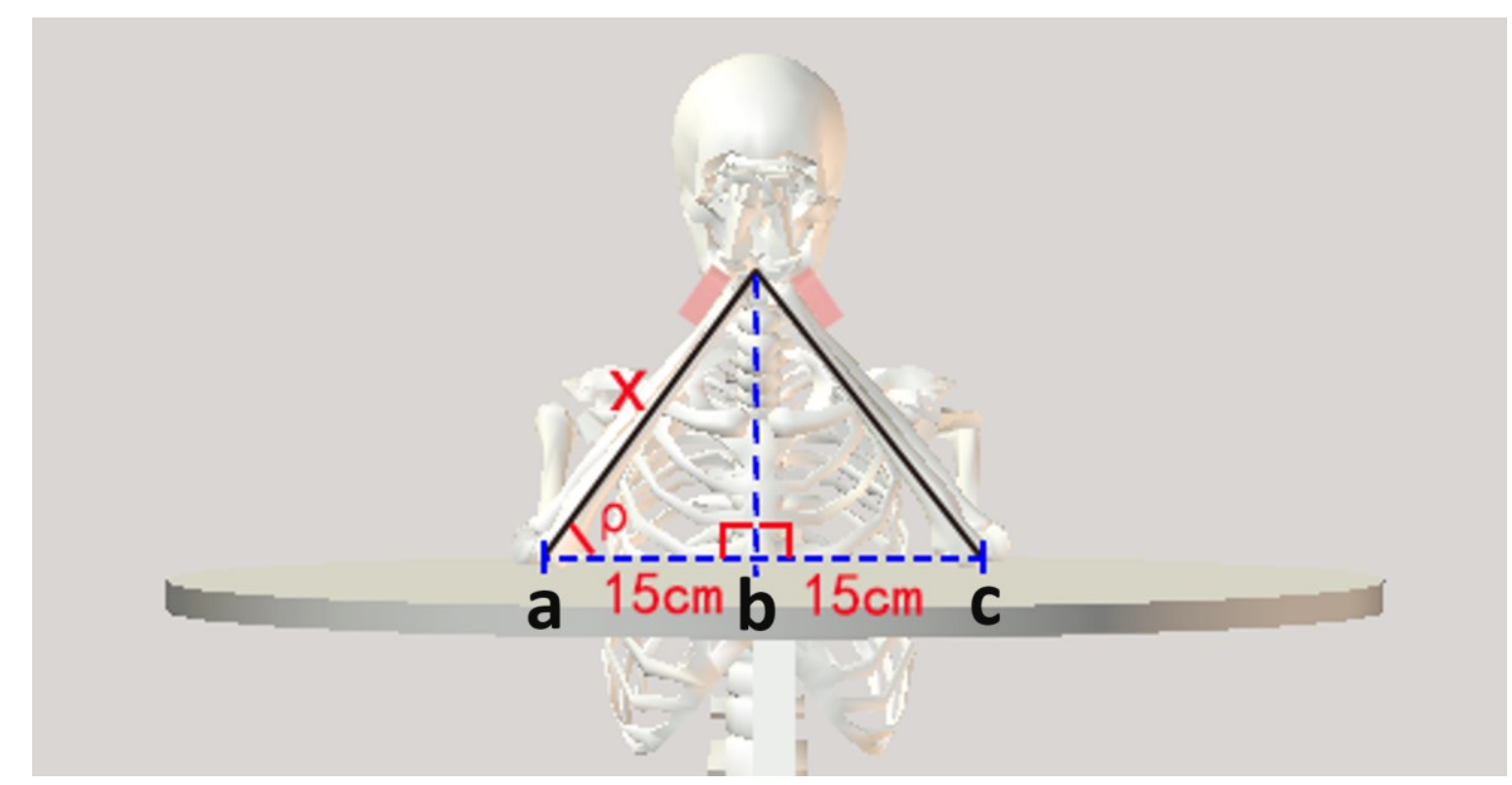

Figure 30. Illustration for the symmetrical requirements of calibration pose performing.

\subsection{Pose Numbers and Pose Design}

When designing the poses for this proposed calibration protocol, the goal is to achieve the optimal design with satisfactory calibration accuracy, shorter calibration time, simpler calibration procedure, and fewer required calibration poses. According to this goal, this proposed calibration protocol consists of four static and easy-to-perform poses, and has been proved to be able to achieve satisfactory accuracy. When designing the calibration poses, it was found to be very difficult to successfully calibrate the main human body parts length with less than four poses. In addition, when reflecting on how to further improve the accuracy of the calibration methodology, increasing the numbers and design of the required calibration poses to realize more delicate tests could be a promising approach. Especially, in this experiment, the calibration accuracy for the upper arm has revealed room for improvement, which partially because, in order to simplify the calibration procedure 
and reduce the numbers of required poses, the design of the upper arm calibration pose and algorithm is based on the previous calibration of another body part. Therefore, improving the design and increasing the numbers of calibration poses to reduce the dependency on other body parts calibration results might be a promising approach to enhance the accuracy of the upper arm length calibration.

\subsection{Sensor Reading Numerical Precision}

In this research, it was found that the calibration algorithm was so sensitive to the sensor reading variations, which means inputting slightly different sensor data may lead to relatively large fluctuations in the error rate. For instance, 0.05-0.1 difference in the sensor reading may lead to the calibration error rate to fluctuate by $3 \%-10 \%$. This situation would typically require the higher reading precision of sensor data to obtain more elaborate calibration results. However, this experiment used the sensor's accompanying data monitor platform on mobile device to wirelessly receive data and support sensor data reading, which is very portable and convenient, but it can only provide the numerical precision of one digit to the right of the decimal point. Therefore, access to the higher numerical precision of sensor data (obtaining more digits to the right of the decimal point) may support further exploration of how the sensor reading numerical precision influences the calibration accuracy, help to obtain more precise calibration results, and be beneficial to more delicately examining the stability and accuracy of the proposed calibration protocol in this research.

\subsection{Calibration Time Consumption}

In this research, the average calibration time duration across all participants is 22.4 minutes, which is a little bit longer than expectations. Ideally, the satisfactory calibration accuracy, simplified calibration procedure, and fast calibration speed are expected to be the advantages of using inertial sensors to calibrate human body structure and size, compared to physically measure. Therefore, to make this inertial sensor-based calibration methodology more practical, the calibration time duration should not exceed the time required to physically measure someone. If commercially applying this calibration methodology in the virtual game context in the future, players might not finish the entire 
calibration process if it would take more than 10 minutes because players might find this process too complicated to complete and may lose their patience.

In analyzing the reasons why it exceeds the expected calibration time duration in this experiment, it may include the following factors.

Firstly, for each calibration pose in this research process, the researcher needed to place the sensor device on the participant's body and ensure the sensor perfectly aligned with the direction of participant's body parts, which would consume certain time.

Secondly, during the calibration process, participants were required to fully understand the requirements of each calibration pose, then performed the pose exactly to comply with the requirements. Calibration poses, such as for forearm or whole leg, are easier to understand and perform perfectly, so it would take less time to calibrate. However, certain calibration pose, such as the upper arm length calibration pose, are relatively more complicated, with more requirements to perform, and sometimes even required the researcher to manually adjust the participant's pose performing to meet the pose requirements, therefore, it would require more calibration time for these poses.

Finally, before reading and recording sensor data, it would also take some time to stabilize the sensor readings.

In future research, it would be beneficial to further explore how to reduce the calibration time, with the premise of maintaining or further improving the calibration accuracy.

\subsection{Participants' Demographics and Influencing Factors}

In this experiment, this proposed calibration methodology was tested on a total of 15 participants, whose ages range from 19 to 56 years old. This group of users was comprised of 10 males and 5 females, and they were all with normal physical conditions.

Among all these demographics factors, age, gender, ethnic are not the influencing factors on the effectiveness and accuracy of this inertial sensor-based human body structure calibration methodology. For instance, in this experiment, different age groups of participants provided similar calibration accuracy, and the 56 years old participant also achieved satisfactory calibration results. 
However, participants' flexibility, fitness, and body shape characteristics are the factors that would influence the calibration results. Even with physically normal conditions, participants' physical flexibility and body coordination degree might be different among all of them, which is nothing to do with age and would result in, for certain poses, some participants might find it difficult to fulfill the pose requirements and others might find the implementation is easy, thus participants' physical flexibility and fitness would have impacts on the calibration accuracy. In addition, participants' body shape characteristics would also be an influencing factor. For slim participants, it would be easier for the researcher to attach sensors to their body to reflect the directions of their body parts compared to participants with huge muscle groups or obese people whose outline of body parts is curved and would be more challenging to achieve the perfect alignment between the directions of sensors and participants' body parts.

\subsection{Humanized Calibration Process}

Although this research is based on a physically normal user group, it is found that each participant's body coordination degree might be different, which would result in not every participant being fit and able to perform every calibration pose, and some calibration poses may be too difficult to perform for some participants, even in physically normal conditions.

Under these circumstances, it may be helpful to design a series of humanized alternative calibration poses for calibrating the same body part, so users could choose their own suitable and comfortable pose to perform according to their own physical conditions, which is to say that by designing various alternative poses, when calibrating exactly the same body part, different users would have the freedom to choose different calibration poses.

By doing so, it would expect to enhance users' satisfaction toward the calibration process through freeing them from the restrictions of the fixed set of calibration poses and offering them the more flexible options of calibration poses, which may also be helpful to achieve higher calibration accuracy. 


\subsection{Sensor Noise}

In the actual application process, each sensor would produce larger or smaller amounts of noise. In this experiment, the sensor noise is within the normal range, which is mainly caused by the following factors. Firstly, the sensor is directly attached to the body of participants, and the human body has a pulse, breathing, and heartbeat, which would result in the less-noticeable body vibrations and may make the sensor readings generate correspondingly slight fluctuations. Secondly, this research was conducted in schools, libraries, or participants' homes, all relatively quiet environments, but there still exist the inevitable vibrations in the environments, such as the operation of machinery and equipment, the movements of surrounding things, etc., which may also produce correspondingly subtle noise readings. In addition, the sensor circuit may also produce a certain noise. In this experiment, due to the fine selection of experimental environments and equipment, the sensor noise is relatively small and within an acceptable range. 


\section{Chapter: Conclusions}

\subsection{Thesis Findings}

In this thesis, a calibration methodology for motion tracking and gesture recognition system has been presented. This proposed calibration protocol is based on the three-axis accelerometer values output by wireless inertial sensors to calibration of players' body parts length (including forearm, upper arm, torso, shinbone, and whole leg) through four easy-to-perform static poses. Aimed at reducing the game control learning time and improve the accuracy of game operation, this calibration method would be expected to be combined with inertial sensor-based motion tracking and gesture recognition system, and to be applied in game context.

Through this experiment, this calibration methodology proved to be a robust approach to calibrate physically normal users' body parts length, with simple calibration pose design, streamlined testing procedures, and satisfactory calibration accuracy $(7.64 \%$ average calibration error rate across all body parts and all participants). However, the calibration time duration is a little bit longer than expected (average 22.4 minutes across all participants).

When considering the calibration accuracy for different body parts, this proposed calibration methodology worked best for the forearm length calibration, followed by the shinbone and the whole leg, and the upper arm length calibration generated relatively more errors (but still within an acceptable range) when compared to other body parts, which is probably due to the upper arm calibration pose being relatively more complicated for participants, as well as the cumulative errors.

When applied on both the left and right body sides to compare and examine its calibration accuracy and stability, the proposed calibration protocol still retained robust performance, and the calibration accuracy of both sides varied within the reasonable range, even if participants' body parts length may not be perfectly symmetrical.

In addition, with the aim of making the calibration process more efficient, effective, user-friendly, and fun, a set of calibration system sample interfaces is proposed, involving all the required poses and requirements designed in this thesis. This calibration system contains a Pose Monitor interface, which can dynamically track users' poses to evaluate if 
their pose meets requirements. Leveraging this Pose Monitor system, users can intuitively understand the requirements of each pose through the dynamical and real-time reflection in the interface, so they could be aware of how to adjust their pose by themselves to meet requirements.

\subsection{Applications}

Today, video games are very popular, especially with the integration of virtual reality, augmented reality, and other cutting-edge technologies with video game design and development. Developing both fun and easy-to-control games is an active research field. Especially in first-person perspective virtual reality video games, players are not able to see their real body when completely immersed in the virtual reality environment, plus the body structure size parameters are different between virtual game characters and players, all of which would increase the complexity of learning game control; therefore, players can learn and grasp the ability to control relatively accurately the virtual reality game characters only through gradual practice and making mistakes.

To solve this problem, the proposed calibration methodology in this research could be perfectly combined with the inertial sensor-based motion tracking and gesture recognition system, which are the important parts of virtual reality video games. This could be realized through calibrating player's body parts length using inertial sensors based on this proposed calibration protocol, and then inputting player's body segments length as parameters into the motion tracking and gesture recognition system to personalize and customize the body structure parameters of a virtual game character to make the virtual game character possess the identical body parts length with the player. By doing so, it may help to reduce the learning time required for players, enhance the efficiency of adapting to game control, improve the accuracy and ease of game operation, and might also indirectly promote fun. 


\subsection{Limitations and Future Work}

While this research has proposed a human body structure calibration methodology using wireless inertial sensors and has proved to be effective, there are still some issues that must be addressed in future research.

One of the issues that needs to improve is the numerical precision of sensor readings. In this research, the calibration algorithm appeared sensitive to sensor reading variations, but the data monitor wireless platform for mobile device, used in this research to receive and read sensor data, can only provide the sensor data numerical precision of one digit to the right of the decimal point. Although based on current sensor data numerical precision, the calibration methodology has already proved to be effective, but it would be beneficial to access higher numerical precision of sensor data in future research (obtaining more digits to the right of the decimal point) to obtain a more precise calibration value of the proposed calibration protocol.

Calibration time duration is another aspect that needs to be enhanced. In this research, the calibration time duration is a little bit longer than expected (average 22.4 minutes), which could be triggered by several factors, including time for sensor placement, time for users to understand and fulfill the pose requirements, etc.. In the future, in order to perfectly integrate this human body structure calibration methodology into the inertial sensor-based motion tracking and gesture recognition system, it would be helpful to explore how to further reduce the calibration time when maintaining or further improving its calibration accuracy.

In addition, even for a physically normal user group, each user's body coordination degree might be different, and not every calibration pose would be appropriate for every user to perform. Therefore, in contrast to restricting users to a set of mandatory calibration poses, it might be another promising direction to explore in future research to design a set of humanized alternative calibration poses for the same body part, and allow users to base on their own physical conditions to freely choose their own suitable calibration poses from multiple options, which would be expected to provide a more flexible and satisfactory calibration user experience, and may help to achieve higher calibration accuracy. 


\section{Appendices}

\section{Appendix A Calibration Protocol Excluded}

This is the calibration protocol excluded in section 3.3.

\section{A.1 Pose for Calibration Shinbone Length (Plus Foot/Shoe Height) in Situation Where Knees Can Touch Together}

This is the calibration protocol excluded in section 3.3.2.

Figure 31 shows the three-perspective simulating figure to illustrate the calibration pose for calculating the shinbone length (plus foot/shoe height) in the situation where knees can touch together. The key points of this pose are listed as below:

- Wearing sensors on both sides of ankles, with the same sensor axes directions shown in Figure 31.

- Sitting down on a chair with both shinbones separated from each other and both feet facing toward the front, and making the distance between both feet equal to $60 \mathrm{~cm}$ (the middle points of both feet serve as the starting point and ending point when measuring the distance between both feet). In so doing, making both knees solidly touch together, making both shinbones perpendicular to the horizontal ground to make $\mathrm{Y}$-axis values of the left and right accelerometer sensors read $\mathrm{Y}=0$ $\mathrm{G}$, and holding this leg position constant.

- $\quad$ Recording $\mathrm{X}$ and $\mathrm{Z}$ axes values of both accelerometer sensors. 

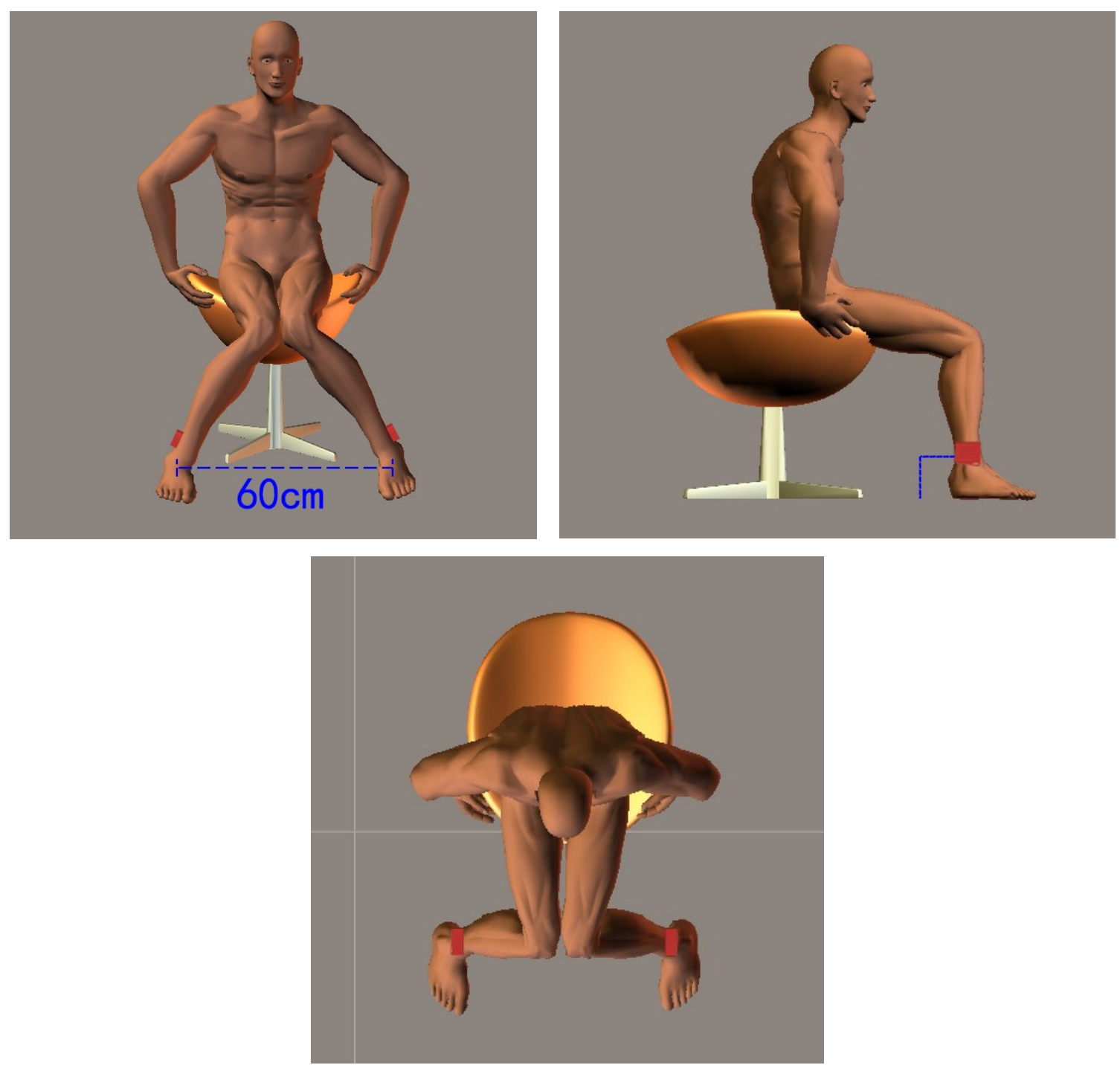

Figure 31. The three-perspective simulating figure to illustrate the calibration pose for calculating the shinbone length (plus foot/shoe height) in the situation where knees can touch together (from the front view, right view, and top view). 


\section{Appendix B Calibration Algorithm Excluded and Supplementary Explanation}

This is the calibration algorithm excluded in section 3.4.

\section{B.1 Calibration Algorithm for Shinbone Length (Plus Foot/Shoe Height) in the Situation Where Knees Can Touch Together}

This is the calibration algorithm excluded in section 3.4.2.

To test the shinbone length (plus foot/shoe height) in the situation where knees can touch together, the angle between the shinbone and the horizontal ground needs to be calculated first. The calculation formulas and details are shown as below.

Wearing sensors on the lateral side of the left and right ankles. When doing this pose, the angle between the leg and the ground is rotated about the Y-axis of the sensors, so the angle is represented as Roll $(\rho)$. When the Y-axis values of the left and right accelerometer sensors read $\mathrm{Y}=0 \mathrm{G}$, the $\mathrm{X}$ and $\mathrm{Z}$ axes values of both accelerometer sensors are used to calculate the angle $\rho$. As shown in Figure 32, $\rho$ represents the angle between the shinbone and the horizontal ground, $\rho$ left represents the angle between the left shinbone and the ground, and $\rho$ right represents the angle between the right shinbone and the ground; $\mathrm{X}$ represents the shinbone length (plus foot/shoe height), $\mathrm{X}_{\text {left }}$ represents the left shinbone length (plus foot/shoe height), and $X_{\text {right }}$ represents the right shinbone length (plus foot/shoe

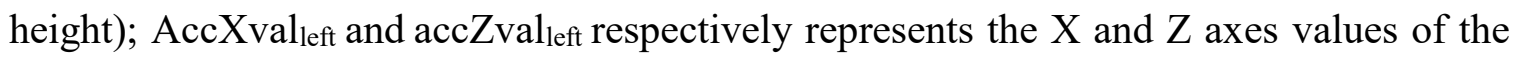
accelerometer sensor worn on the left ankle, and accXval ${ }_{\text {right }}$ and accZval ${ }_{\text {right }}$ respectively represent the $\mathrm{X}$ and $\mathrm{Z}$ axes values of the accelerometer sensor worn on the right ankle. Therefore, the calibration algorithms are shown as Equation (12) and (13):

For calculating left shinbone length (plus foot/shoe height),

$$
\begin{aligned}
\rho_{\text {left }} & =\Pi-\operatorname{atan} 2\left(\operatorname{accXval}_{\text {left }}, \operatorname{accZval}_{\text {left }}\right) \\
& =-\arctan \left(\operatorname{accXval_{left}} / \operatorname{accZval}_{\text {left }}\right) \\
X_{\text {left }} & =30 \mathrm{~cm} / \cos \rho \text { left }
\end{aligned}
$$

For calculating right shinbone length (plus foot/shoe height),

$$
\begin{aligned}
& \rho_{\text {right }}=\Pi-\operatorname{atan} 2\left(\operatorname{accXval}_{\text {right}}, \text { accZval }_{\text {right }}\right) \\
& =-\arctan \left(\operatorname{accXval}_{\text {right }} / \operatorname{accZval}_{\text {right }}\right) \\
& X_{\text {right }}=30 \mathrm{~cm} / \cos \rho \text { right }
\end{aligned}
$$




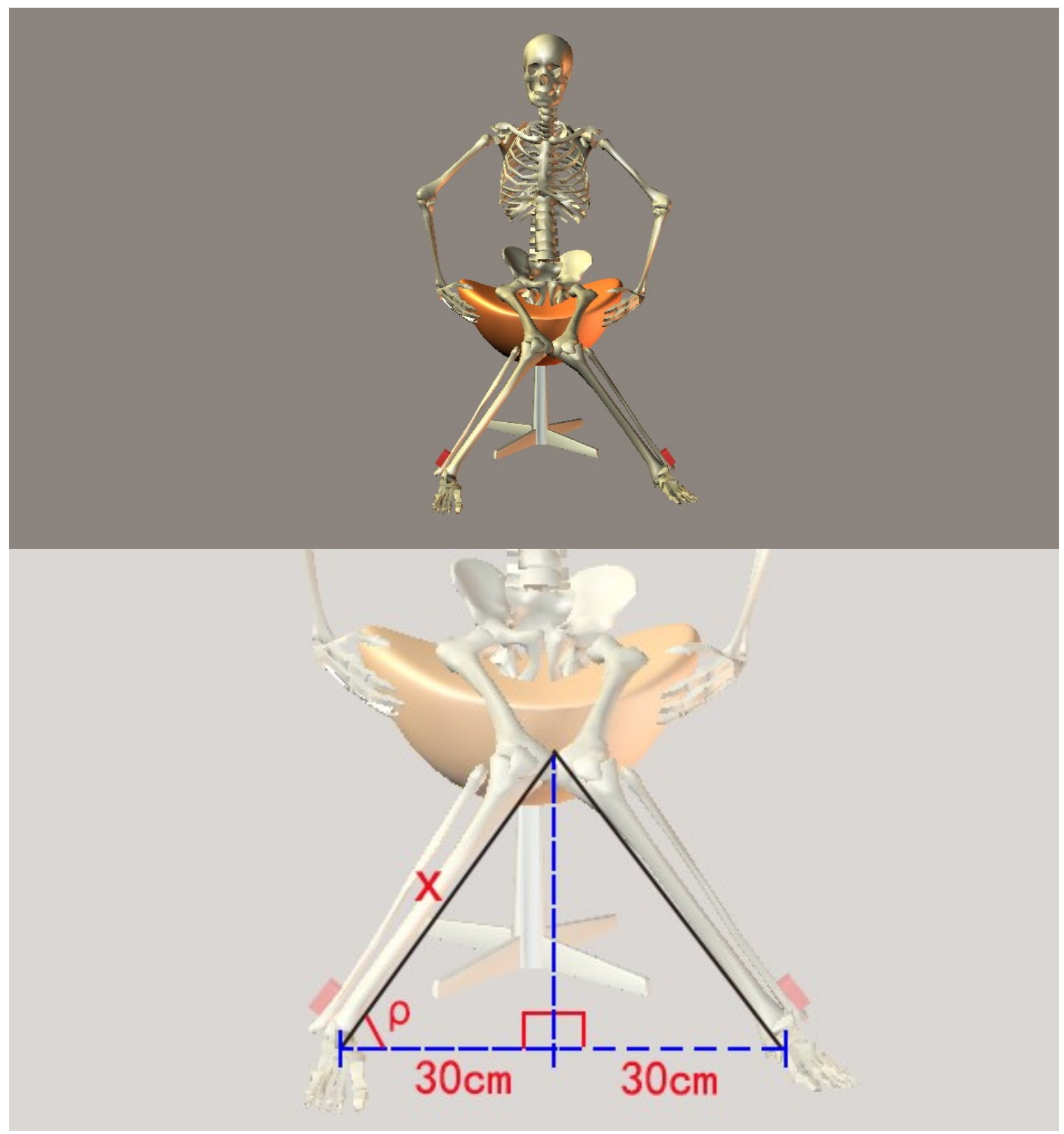

Figure 32. Skeletal model for explaining calibration algorithm for the shinbone length (plus foot/shoe height) in the situation where knees can touch together.

\section{B.2 Verification of Combining Algorithm of Shinbone Length (Plus Foot/Shoe}

Height) for Both Situations: Knees Touch Together \& Knees Cannot Touch

This is the calibration algorithm combination verification excluded in section 3.4.2.

When considering the calculation formulas of the two situations (knees can touch together, and knees cannot touch), the calculation formulas of the situation where knees cannot touch can be used to calculate the situation of knees touching together. In the 
formulas where knees cannot touch, $\mathrm{d}$ represents the distance between both knees and the unit is in centimeters $(\mathrm{cm})$, and when knees can touch together, the distance between both knees is zero, so $d=0 \mathrm{~cm}$. Therefore, in the situation where knees can touch together, one may input $\mathrm{d}=0 \mathrm{~cm}$ into the formulas where knees cannot touch, so the formulas where knees cannot touch becomes exactly the same as the formulas where knees can touch together, as shown in Equation (14) and (15). Therefore, in order to simplify the calculation protocol and algorithm for calculating the shinbone length (plus foot/shoe height), the formulas where knees cannot touch can be used to calculate both situations (knees can touch together, and knees cannot touch):

For calculating left shinbone length (plus foot/shoe height) in the situation where knees can touch together, using the algorithm where knees cannot touch,

$$
\begin{aligned}
& \rho_{\text {left }}=\Pi-\operatorname{atan} 2\left(\operatorname{accXval}_{\text {left, }}, \operatorname{accZval}_{\text {left }}\right) \\
& =-\arctan \left(\mathrm{accXval}_{\text {left }} / \mathrm{accZval}_{\text {left }}\right) \\
& X_{\text {left }}=[(30 \mathrm{~cm}-d / 2) / 30 \mathrm{~cm}]^{*}\left[30 \mathrm{~cm} / \cos \rho_{\text {left }}\right] \\
& =[(30 \mathrm{~cm}-0 \mathrm{~cm} / 2) / 30 \mathrm{~cm}]^{*}\left[30 \mathrm{~cm} / \cos \rho_{\text {left }}\right] \\
& =30 \mathrm{~cm} / \cos \rho_{\text {left }}
\end{aligned}
$$

For calculating right shinbone length (plus foot/shoe height) in the situation where knees can touch together, using the algorithm where knees cannot touch,

$$
\begin{aligned}
\rho_{\text {right }} & =\Pi-\operatorname{atan} 2\left(\operatorname{accXval}_{\text {right }}, \operatorname{accZval}_{\text {right }}\right) \\
& =-\arctan \left(\operatorname{accXval} 1_{\text {right }} / \operatorname{accZval}_{\text {right }}\right) \\
X_{\text {right }} & =[(30 \mathrm{~cm}-\mathrm{d} / 2) / 30 \mathrm{~cm}]^{*}\left[30 \mathrm{~cm} / \cos \rho_{\text {right }}\right] \\
& =[(30 \mathrm{~cm}-0 \mathrm{~cm} / 2) / 30 \mathrm{~cm}]^{*}\left[30 \mathrm{~cm} / \cos \rho_{\text {right }}\right] \\
& =30 \mathrm{~cm} / \cos \rho_{\text {right }}
\end{aligned}
$$




\section{Appendix C Calibration Results Excluded}

This is the calibration results excluded in Chapter 4

\section{C.1 Shinbone Length Calibration Results Excluded}

This is the P8's shinbone calibration results excluded in section 4.2.5.

Table 11. P8's shinbone length (plus foot/shoe height) calibration detailed data, including calculated length $(\mathrm{cm})$, real length $(\mathrm{cm})$, and error rates $(\%)$.

\begin{tabular}{|l|c|c|}
\hline \multicolumn{3}{|c|}{ P8 Shinebone Calibration } \\
\hline Shinbone & Left & Right \\
\hline Error Rate (\%) & $31.76 \%$ & $31.09 \%$ \\
\hline Calculated length $(\mathrm{cm})$ & 34.8 & 34.8 \\
\hline Real length $(\mathrm{cm})$ & 51.0 & 50.5 \\
\hline
\end{tabular}




\section{References}

[1] F. W. Booth, S. E. Gordon, C. J. Carlson, M. T. Hamilton, W. Frank, S. E. Gordon, and C. J. Carlson, "Waging war on modern chronic diseases: primary prevention through exercise biology," J. Appl. Physiol., vol. 8, no. 2, pp. 774-787, 2000 .

[2] Y. Jung and B. Cha, "Gesture Recognition Based on Motion Inertial Sensors for Ubiquitous Interactive Game Contents," IETE Tech. Rev., vol. 27, no. 2, 2010.

[3] D. Arsenault, "A Quaternion-Based Motion Tracking and Gesture Recognition System Using Wireless Inertial Sensors," 2014.

[4] J. A. Pineda, D. S. Silverman, A. Vankov, and J. Hestenes, "Learning to Control Brain Rhythms: Making a Brain-Computer Interface Possible," IEEE Trans. Neural Syst. Rehabil. Eng., vol. 11, no. 2, pp. 181-184, 2003.

[5] G. Müller-putz, R. Scherer, and G. Pfurtscheller, "Game-like Training to Learn Single Switch Operated Neuroprosthetic Control," BRAINPLAY 07 BrainComputer Interfaces Games Work. ACE (Advances Comput. Entertain.), vol. 4, 2007.

[6] S. Masuko and J. Hoshino, “A Fitness Game Reflecting Heart Rate," Proc. 2006 ACM SIGCHI Int. Conf. Adv. Comput. Entertain. Technol., 2006.

[7] K. M. Gilleade, A. Dix, and J. Allanson, "Affective Videogames and Modes of Affective Gaming: Assist Me, Challenge Me, Emote Me," Proc. DiGRA 2005 Conf. Chang. Views-Worlds Play, 2005.

[8] L. Nacke, M. Kalyn, C. Lough, and R. Mandryk, "Biofeedback game design: using direct and indirect physiological control to enhance game interaction," Proc. SIGCHI, pp. 103-112, 2011.

[9] K. Kuikkaniemi, T. Laitinen, M. Turpeinen, T. Saari, and I. Kosunen, "The Influence of Implicit and Explicit Biofeedback in First-Person Shooter Games," Proc. 28th Int. Conf. Hum. factors Comput. Syst., pp. 859-868, 2010.

[10] M. Chen, S. Gonzalez, A. Vasilakos, H. Cao, and V. C. M. Leung, "Body Area Networks : A Survey," Mob. Networks Appl., vol. 16, no. 2, pp. 171-193, 2011.

[11] A. Nijholt, D. P. Bos, and B. Reuderink, "Turning shortcomings into challenges : Brain - computer interfaces for games," Entertain. Comput., vol. 1, no. 2, pp. 8594, 2009.

[12] W. Tao, T. Liu, R. Zheng, and H. Feng, "Gait Analysis Using Wearable Sensors," 
Sensors, vol. 12, pp. 2255-2283, 2012.

[13] A. Muro-de-la-herran, B. Garcia-zapirain, and A. Mendez-zorrilla, "Gait Analysis Methods: An Overview of Wearable and Non-Wearable Systems, Highlighting Clinical Applications," Sensors, vol. 14, no. 2, pp. 3362-3394, 2014.

[14] L. S. Vargas-Valencia, A. Elias, E. Rocon, T. Bastos-Filho, and A. Frizera, "An IMU-to-Body Alignment Method Applied to Human Gait Analysis," Sensors, vol. 16, no. 12, pp. 1-17, 2016.

[15] K. Hashimoto, K. Higuchi, Y. Nakayama, and M. Abo, "Ability for Basic Movement as an Early Predictor of Functioning Related to Activities of Daily Living in Stroke Patients," Neurorehabil. Neural Repair, vol. 21, no. 4, pp. $353-$ 357, 2007.

[16] S. Zhou, Q. Shan, F. Fei, W. J. Li, C. P. Kwong, P. C. K. Wu, B. Meng, C. K. H. Chan, and J. Y. J. Liou, "Gesture Recognition for Interactive Controllers Using MEMS Motion Sensors," 2009 4th IEEE Int. Conf. Nano/Micro Eng. Mol. Syst., pp. 935-940, 2009.

[17] N. Crampton, K. Fox, H. Johnston, and A. Whitehead, "Dance, Dance Evolution : Accelerometer Sensor Networks as Input to Video Games ionjgames ithals ptecno diameter," 2007 IEEE Int. Work. Haptic, Audio Vis. Environ. Games, 2007.

[18] P. Keir, J. Payne, J. Elgoyhen, M. Horner, M. Naef, and P. Anderson, "Gesturerecognition with Non-referenced Tracking," IEEE Symp. 3D User Interfaces, 2006. 3DUI 2006., 2006.

[19] S. You, U. Neumann, and R. Azuma, "Hybrid Inertial and Vision Tracking for Augmented Reality Registration," Proc. IEEE Virtual Real. (Cat. No. 99CB36316).

[20] S. Giannarou, Z. Zhang, and G. Yang, "Deformable Structure From Motion by Fusing Visual and Inertial Measurement Data," 2012 IEEE/RSJ Int. Conf. Intell. Robot. Syst., pp. 4816-4821, 2012.

[21] S. Mitra and T. Acharya, "Gesture Recognition: A Survey," IEEE Trans. Syst. Man, Cybern. Part C (Applications Rev.), vol. 37, no. 3, pp. 311-324, 2007.

[22] T. J. Broida, S. Chandrashekhar, and R. Chellappa, "Recursive 3-D Motion Estimation from Monocular Image Sequence," IEEE Trans. Aerosp. Electron., vol. 26, no. 4, 1990.

[23] W. T. Freeman and C. D. Weissman, "Television Control by Hand Gestures," IEEE Int. Work. Autom. Face Gestures Recogntion, 1995.

[24] K. Abe, H. Saito, and S. Ozawa, "3-D Drawing System via Hand Motion Recgriition from Two Cameras 3-D Drawing System," IEEE Int. Conf. Syst. Man 
Cybern., vol. 2, pp. 840-845, 2000.

[25] W. Bang, W. Chang, K. Kang, E. Choi, A. Potanin, and D. Kim, "Self-contained Spatial Input Device for Wearable Computers," 7 th IEEE Int. Symp. Wearable Comput., pp. 26-34, 2003.

[26] Y. Yi, "On Improving the Accuracy and Reliability of GPS/INS-Based Direct Sensor Georeferencing," 2007.

[27] W. H. Baird, "An introduction to inertial navigation," Am. J. Phys., vol. 77, no. 9, 2009.

[28] J. Wang and F. Chuang, "An Accelerometer-Based Digital Pen With a Trajectory Recognition Algorithm for Handwritten Digit and Gesture Recognition," IEEE Trans. Ind. Electron., vol. 59, no. 7, pp. 2998-3007, 2012.

[29] S. Zhou, F. Fei, G. Zhang, Y. Liu, and W. J. Li, "Hand-Writing Motion Tracking with Vision-Inertial Sensor Fusion: Calibration and Error Correction," Sensors, vol. 14, no. 9, pp. 15641-15657, 2014.

[30] L. Ricci, D. Formica, L. Sparaci, F. R. Lasorsa, F. Taffoni, E. Tamilia, and E. Guglielmelli, "A New Calibration Methodology for Thorax and Upper Limbs Motion Capture in Children Using Magneto and Inertial Sensors," Sensors, vol. 14, pp. 1057-1072, 2014.

[31] S. Zhou, F. Fei, G. Zhang, J. D. Mai, Y. Liu, J. Y. J. Liou, and W. J. Li, “2D Human Gesture Tracking and Recognition by the Fusion of MEMS Inertial and Vision Sensors," IEEE Sens. J., vol. 14, no. 4, pp. 1160-1170, 2014.

[32] D. Hyoung and W. Jang, "Automation in Construction Utilization of ubiquitous computing for construction AR technology," Autom. Constr., vol. 18, no. 8, pp. 1063-1069, 2009.

[33] G. Welch and E. Foxlin, "Motion Tracking : No Silver Bullet, but a Respectable," IEEE Comput. Graph. Appl., vol. 22, no. 6, pp. 24-38, 2002.

[34] C. Hu, M. Li, S. Song, W. Yang, R. Zhang, and M. Q. Meng, "A Cubic 3-Axis Magnetic Sensor Array for Wirelessly Tracking Magnet Position and A Cubic 3Axis Magnetic Sensor Array for Wirelessly Tracking Magnet Position and Orientation," IEEE Sens. J., vol. 10, no. 5, pp. 903-913, 2010.

[35] B. M. Aron, G. Simon, and M. Berger, "Use of inertial sensors to support," Comput. Animat. Virtual Worlds, vol. 18, no. 1, pp. 57-68, 2007.

[36] P. Saeedi, P. D. Lawrence, and D. G. Lowe, "Vision-Based 3-D Trajectory Tracking for Unknown Environments," IEEE Trans. Robot., vol. 22, no. 1, pp. 
119-136, 2006.

[37] R. Xu, S. Zhou, and W. J. Li, "MEMS Accelerometer Based Nonspecific-User," IEEE Sens. J., vol. 12, no. 5, pp. 1166-1173, 2012.

[38] R. Z. Khan and N. A. Ibraheem, "Comparative Study of Hand Gesture Recognition System," Comput. Sci. Inf. Technol., vol. 2, no. 3, 2012.

[39] W. H. K. De Vries, H. E. J. Veeger, C. T. M. Baten, and F. C. T. Van Der Helm, "Gait \& Posture Magnetic distortion in motion labs, implications for validating inertial magnetic sensors," Gait Posture, vol. 29, no. 4, pp. 535-541, 2009.

[40] N. H. Q. Phuong, H.-J. Kang, Y.-S. Suh, and Y.-S. Ro, “A DCM Based Orientation Estimation Algorithm with an Inertial Measurement Unit and a Magnetic Compass," J. Univers. Comput. Sci., vol. 15, no. 4, 2009.

[41] H. M. Schepers, D. Roetenberg, and P. H. Veltink, "Ambulatory human motion tracking by fusion of inertial and magnetic sensing with adaptive actuation," Med. Biol. Eng. Comput., vol. 48, no. 1, pp. 27-37, 2010.

[42] A. R. Jim, F. Seco, C. Prieto, J. Guevara, and A. I. M. U. Description, "A Comparison of Pedestrian Dead-Reckoning Algorithms using a Low-Cost MEMS IMU,” 2009 IEEE Int. Symp. Intell. Signal Process., pp. 37-42, 2009.

[43] M. Achtelik, T. Zhang, K. Kiihnlenz, and M. Buss, "Visual Tracking and Control of a Quadcopter Using a Stereo Camera System and Inertial Sensors," 2009 Int. Conf. Mechatronics, pp. 2863-2869, 2009.

[44] G. Bleser and D. Stricker, "Computers \& Graphics Advanced tracking through efficient image processing and visual - inertial sensor fusion," Comput. Graph., vol. 33, no. 1, pp. 59-72, 2009.

[45] G. Bleser and G. Hendeby, "Using optical flow for filling the gaps in visualinertial tracking," 2010 18th Eur. Signal Process. Conf., 2010.

[46] G. Welch and G. Bishop, "An Introduction to the Kalman Filter," 2001.

[47] J. A. Corrales, F. A. Candelas, and F. Torres, "Kalman Filtering for Sensor Fusion in a Human Tracking System Kalman Filtering for Sensor Fusion in a Human Tracking System," Kalman Filter, 2010.

[48] D. Dusha and L. Mejias, "Error analysis and attitude observability of a monocular GPS/visual odometry integrated navigation filter," Int. J. Rob. Res., vol. 31, no. 6, pp. 714-737, 2012.

[49] F. Aparicio, J. Zato, J. E. Naranjo, and F. Jime, "GPS and Inertial Systems for 
High Precision Positioning on Motorways," J. Navig., pp. 351-363, 2009.

[50] C. H. Kang, S. Y. Kim, and C. G. Park, "Improvement of a Low Cost MEMS Inertial-GPS Integrated System Us- ing Wavelet Denoising Techniques," Int. J. Aeronaut. Sp. Sci., vol. 12, no. 4, pp. 371-378, 2011.

[51] R. E. Hopkins, N. M. Barbour, D. E. Gustafson, and P. Sherman, "Miniature Inertial and Augmentation Sensors for Integrated Inertial/GPS Based Navigation Applications," 2010.

[52] J. Hol, Sensor Fusion and Calibration of Inertial Sensors, Vision, Ultra-Wideband and GPS. Linköping: Department of Electrical Engineering, Linköping University, 2011.

[53] A. M. Sabatini, C. Martelloni, S. Scapellato, and F. Cavallo, "Assessment of Walking Features From Foot Inertial Sensing,” vol. 52, no. 3, pp. 486-494, 2005.

[54] M. Boutaayamou, C. Schwartz, J. Stamatakis, V. Denoël, D. Maquet, B. Forthomme, J. Croisier, B. Macq, J. G. Verly, G. Garraux, and O. Brüls, "Development and validation of an accelerometer-based method for quantifying gait events," vol. 37, pp. 226-232, 2015.

[55] A. Rampp, J. Barth, and S. Sch, "Inertial Sensor-Based Stride Parameter Calculation From Gait Sequences in Geriatric Patients," vol. 62, no. 4, pp. 1089 1097, 2015.

[56] P. Picerno, V. Camomilla, and L. Capranica, "Countermovement jump performance assessment using a wearable 3D inertial measurement unit," J. Sports Sci., vol. 29, no. 2, 2011.

[57] Z. Lin, M. Uemura, M. Zecca, S. Sessa, H. Ishii, M. Tomikawa, M. Hashizume, and A. Takanishi, "Objective Skill Evaluation for Laparoscopic Training Based on Motion Analysis," IEEE Trans. Biomed. Eng., vol. 60, no. 4, pp. 977-985, 2013.

[58] J. Favre, R. Aissaoui, B. M. Jolles, J. A. De Guise, and K. Aminian, "Functional calibration procedure for 3D knee joint angle description using inertial sensors," $J$. Biomech., vol. 42, no. 14, pp. 2330-2335, 2009.

[59] A. G. Cutti, A. Ferrari, P. Garofalo, M. Raggi, A. Cappello, and A. Ferrari, "'Outwalk': a protocol for clinical gait analysis based on inertial and magnetic sensors," Med. Biol. Eng. Comput., vol. 48, no. 1, pp. 17-25, 2010.

[60] E. Palermo, S. Rossi, F. Marini, F. Patanè, and P. Cappa, "Experimental evaluation of accuracy and repeatability of a novel body-to-sensor calibration procedure for inertial sensor-based gait analysis," Measurement, vol. 52, pp. 145-155, 2014.

[61] W. Kong, S. Sessa, M. Zecca, and A. Takanishi, "Anatomical Calibration through Post-Processing of Standard Motion Tests Data," Sensors, vol. 16, no. 12, p. 2011, 
2016.

[62] H. Zhou and H. Hu, "Human motion tracking for rehabilitation-A survey," Biomed. Signal Process. Control, vol. 3, no. 1, pp. 1-18, 2008.

[63] C. Wong, Z. Zhang, B. Lo, and G. Yang, "Wearable Sensing for Solid Biomechanics : A Review," vol. 15, no. 5, pp. 2747-2760, 2015.

[64] Z. F. Syed, P. Aggarwal, X. N. C Goodall, and N. El-Sheimy, "A new multiposition calibration method for MEMS inertial navigation systems," Meas. Sci. Technol., vol. 18, no. 7, pp. 1897-1907, 2007.

[65] I. Frosio, F. Pedersini, N. A. Borghese, and A. S. Model, "Autocalibration of MEMS Accelerometers," IEEE Trans. Instrum. Meas., vol. 58, no. 6, pp. 2034 2041, 2009.

[66] P. Petkov and T. Slavov, "Stochastic Modeling of MEMS Inertial Sensors," Cybern. Inf. Technol., vol. 10, no. 2, pp. 31-40, 2010.

[67] J. Lee, S. Hong, N. Moon, and S. Oh, "Acoustic Sensor-Based Multiple Object Tracking with Visual Information Association," EURASIP J. Adv. Signal Process., 2010.

[68] N. El-sheimy, H. Hou, and X. Niu, "Analysis and Modeling of Inertial Sensors Using Allan Variance," vol. 57, no. 1, pp. 140-149, 2008.

[69] T. Seel, J. Raisch, and T. Schauer, "IMU-Based Joint Angle Measurement for Gait Analysis," Sensors (Basel)., vol. 14, no. 4, pp. 6891-6909, 2014.

[70] A. Cereatti, D. Trojaniello, U. Della Croce, P. Sciences, and C. Sciences, "Accurately measuring human movement using magneto-inertial sensors: techniques and challenges," Proc. 2015 IEEE Int. Symp. Inert. Sensors Syst., pp. $1-4,2015$.

[71] P. Picerno, A. Cereatti, and A. Cappozzo, "Joint kinematics estimate using wearable inertial and magnetic sensing modules," vol. 28, pp. 588-595, 2008.

[72] D. Roetenberg, H. Luinge, and P. Slycke, "Xsens Mvn: Full 6d of Human Motion Tracking Using Miniature Inertial Sensors," pp. 1-9, 2009.

[73] H. J. Luinge, P. H. Veltink, and C. T. M. Baten, "Ambulatory measurement of arm orientation," vol. 40, pp. 78-85, 2007.

[74] J. F. Wagner, "Adapting the Principle of Integrated Navigation Systems to Measuring the Motion of Rigid Multibody Systems," pp. 87-110, 2004.

[75] M. Miezal, B. Taetz, and G. Bleser, "On Inertial Body Tracking in the Presence of 
Model Calibration Errors,” Sensors (Basel)., vol. 16, no. 7, p. 1132, 2016.

[76] K. J. O. Donovan, R. Kamnik, D. T. O. Keeffe, and G. M. Lyons, "An inertial and magnetic sensor based technique for joint angle measurement," vol. 40, pp. 2604$2611,2007$.

[77] J. Favre, B. M. Jolles, R. Aissaoui, and K. Aminian, "Ambulatory measurement of 3D knee joint angle," J. Biomech., vol. 41, no. 5, pp. 1029-1035, 2008.

[78] H. Yang and J. Ye, "A Calibration Process for Tracking Upper Limb Motion with Inertial Sensors," 2011 IEEE Int. Conf. Mechatronics Autom., 2011.

[79] J. C. Van den Noort, S. H. Wiertsema, K. M. C. Hekman, C. P. Schönhuth, J. Dekker, and J. Harlaar, "Reliability and precision of 3D wireless measurement of scapular kinematics," Med. Biol. Eng. Comput., vol. 52, no. 11, pp. 921-931, 2014.

[80] A. Steed, S. Frlston, M. M. Lopez, J. Drummond, Y. Pan, and D. Swapp, “An 'In the Wild' Experiment on Presence and Embodiment using Consumer Virtual Reality Equipment," IEEE Transactions on Visualization and Computer Graphics, vol. 22, no. 4, pp. 1406-1414, Apr. 2016.

[81] Q. Lin, J. J. Rieser, and B. Bodenheimer, "Stepping off a ledge in an HMD-based immersive virtual environment," Proceedings of the ACM Symposium on Applied Perception - SAP'13, pp. 107-110, 2013. 